\title{
Determination of Radionuclides in Biological Samples by Liquid and Solid Phase Extraction.
}

\author{
By
}

\section{Raymond Ko}

\begin{abstract}
A thesis submitted to the Faculty of Graduate and Postdoctoral Affairs in partial fulfillment of the requirements for the degree of

Master of Science

in

Chemistry
\end{abstract}

Carleton University

Ottawa, Ontario

(C) 2016 Raymond Ko 


\begin{abstract}
Radionuclide separation and preconcentration methods were developed for two types of biological samples that play a role in assessing internal radiation contamination. For the determination of $\mathrm{Pu}-238$ in urine, either as a single isotope or in a mixture, a dispersive liquid-liquid microextraction technique was used to extract them from the samples. Parameters including dispersers, extractants, back extractants and $\mathrm{pH}$ were optimized to achieve high recoveries of plutonium. Liquid scintillation counting was used for samples containing only plutonium, and alpha spectroscopy was used for samples containing a mixture of isotopes. For the determination of Am-241 in a humerus from a deceased worker exposed to americium 40 years prior, subsamples were taken by shaving successive layers off the bone (from the periosteum to the marrow) for dry/wet ashing before americium was isolated by extraction chromatography and determined using alpha spectrometry. The results showed americium retention and migration throughout the total bone volume.
\end{abstract}




\section{Acknowledgement}

I would like to express my sincere appreciation to my supervisors Dr. Edward Lai, and Dr. Chunsheng Li for their guidance, and wisdom.

I would also like to thank the undergrad students who assisted me: Amelia M, Krysta T, and Dominique A. I would also like to thank Dr. Baki Sadi for his technical expertise.

Finally, I would like to thank my parents for their kindness and support. 


\section{Table of Contents}

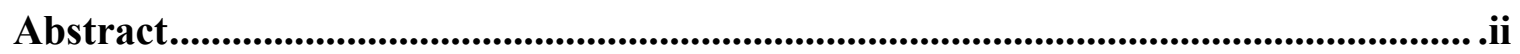

Acknowledgements ....................................................................................................................... iii

Table of Contents ....................................................................................................... iv

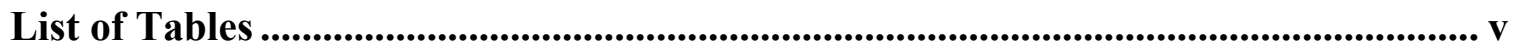

List of Illustrations............................................................................................... vi

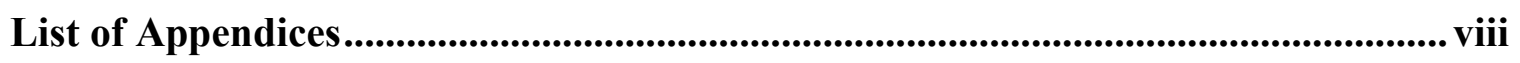

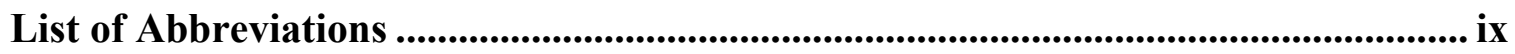

\section{Introduction.}

2 Part A: Determination of Plutonium 238 in urine samples by dispersive liquidliquid microextraction and liquid scintillation counting or alpha spectroscopy......... 4

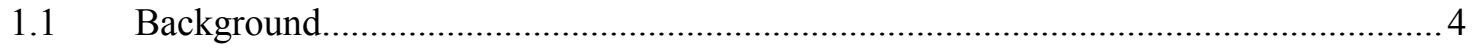

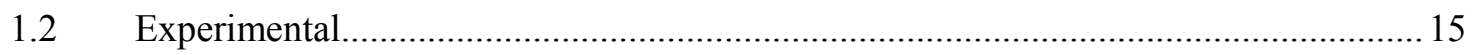

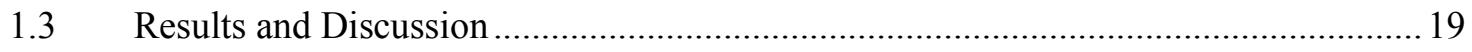

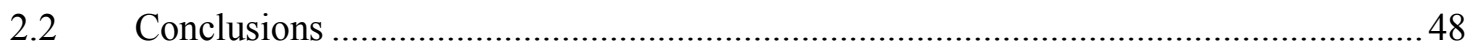

3 Part B: Determination of Americium 241 distribution in bone samples by extraction chromatography and alpha spectroscopy

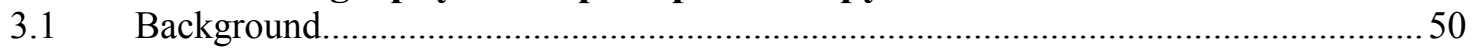

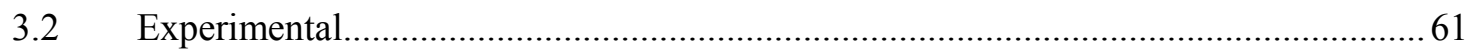

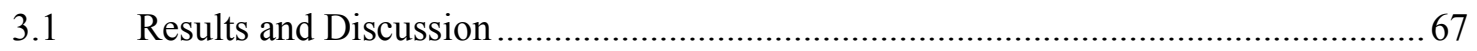

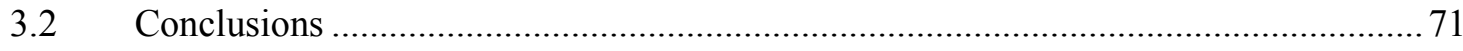

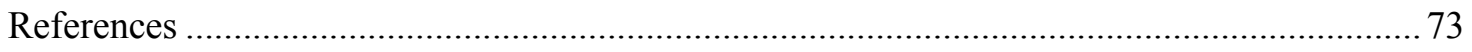

Appendix

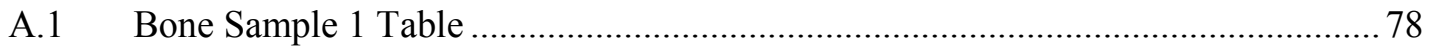

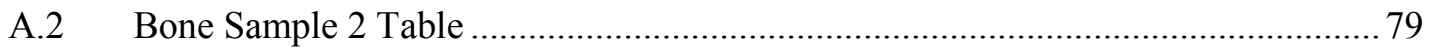




\section{List of Tables}

Table 1: Actinides and their alpha decay energies................................................... 7

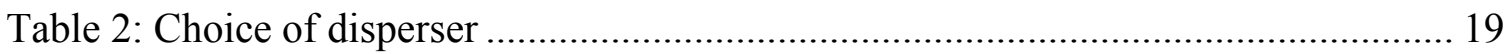

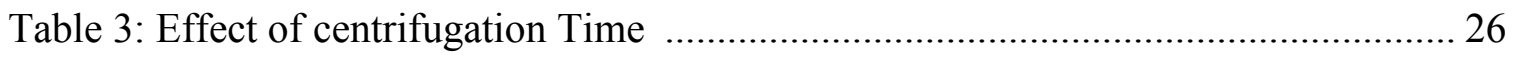

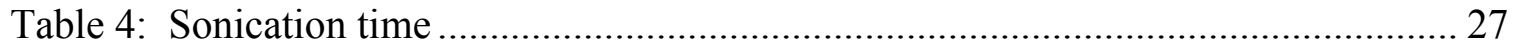

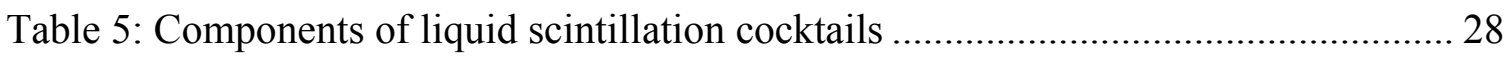

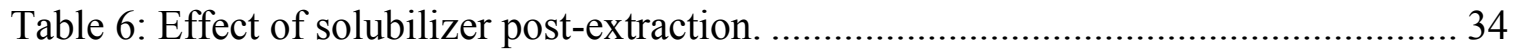

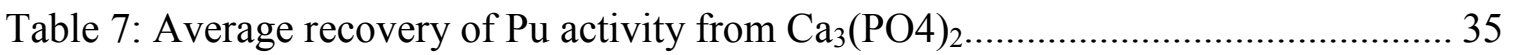

Table 8 Sequential back extraction recoveries for urine containing both $\mathrm{Pu}$ and $\mathrm{Am}$.......42

Table 9: Effect of Fenton's reagent on recovery of Pu-DTPA ....................................... 42

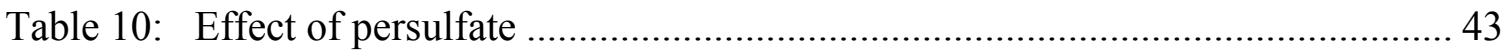

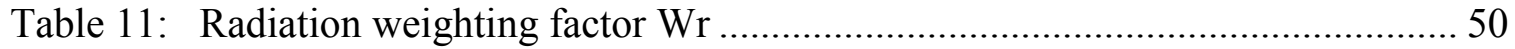

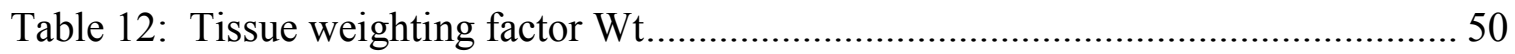




\section{List of Figures}

Figure 1: Structure of HDEHP................................................................................. 10

Figure 2: Effect of methanol volume on the recovery of plutonium. ........................... 20

Figure 3: Effect of tetrachloroethylene on recovery of plutonium .............................. 21

Figure 4: Effect of HDEHP volume on extraction...................................................... 22

Figure 5: Effect of $\mathrm{pH}$ on recovery of plutonium .................................................. 24

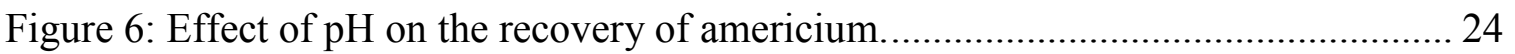

Figure 7: Effect of $\mathrm{pH}$ on the recovery of uranium. .............................................. 25

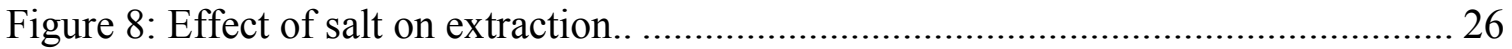

Figure 9: Effect of delayed counting in different LSC cocktails................................ 29

Figure 10: Effect of delayed counting in HiSafe 3 and Ultima Gold AB....................... 30

Figure 11: Effect of tetrachloroethylene on quenching parameter tSIE ........................ 31

Figure 12: Effect of concentrated nitric acid on quenching parameter tSIE................... 32

Figure 13: Effect of a concentrated matrix on quenching parameter tSIE. ..................... 32

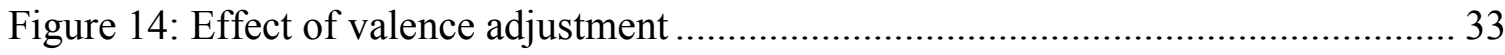

Figure 15. Back Extraction of Pu-HDEHP from phosphoric acid media....................... 36

Figure 16: Back extraction of Plutonium from HDEHP ......................................... 37

Figure 17: Alpha spectrum for back extraction using 2M HCl and 0.2M ascorbic.......... 38

Figure 18: Alpha Spectrum for back extraction with 0.2M oxalate. ........................... 38

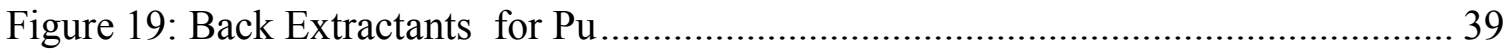

Figure 20: Back extraction of Am from nitric acid media ....................................... 40

Figure 21: Back extraction of $\mathrm{Pu}$ from nitric acid media........................................... 41

Figure 22: Back extraction of $\mathrm{Pu}$ and $\mathrm{Am}$ using $0.5 \mathrm{M} \mathrm{HCl}$ and varying $\left[\mathrm{NaNO}_{2}\right] \ldots \ldots \ldots . . .41$ 
Figure 23: Replicate samples from eight urine types............................................ 44

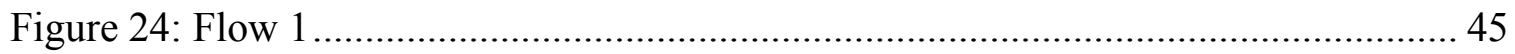

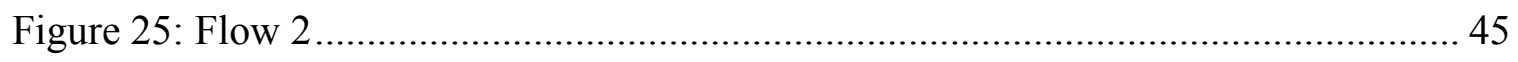

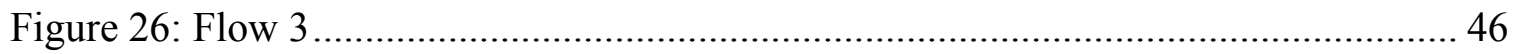

Figure 27: Calculation route from intake to dose .................................................. 47

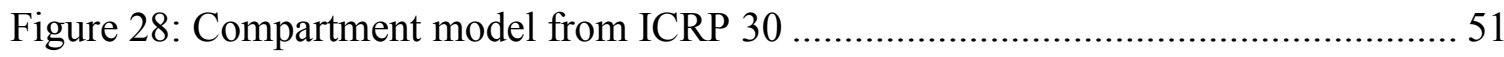

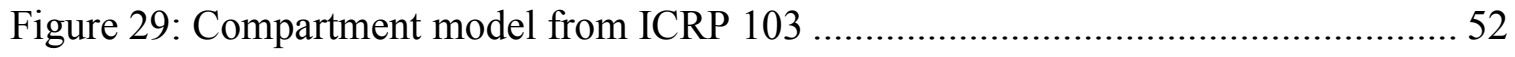

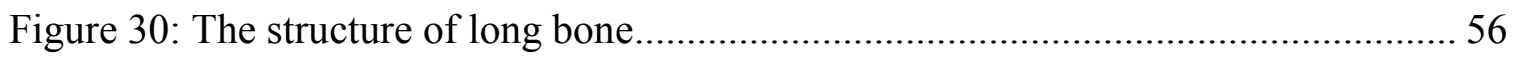

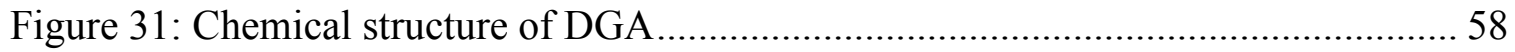

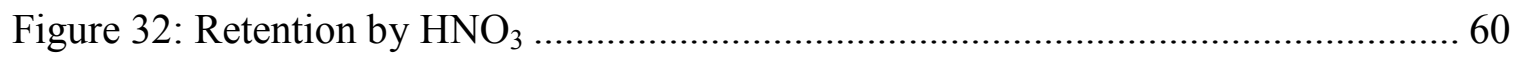

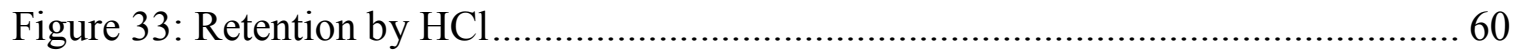

Figure 34: MX grafter tool, with chamber shown in inset.................................62

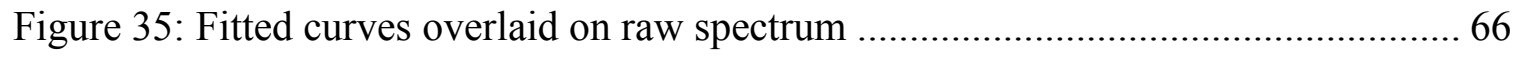

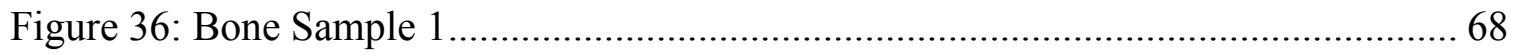

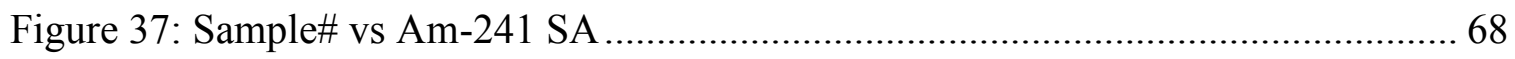

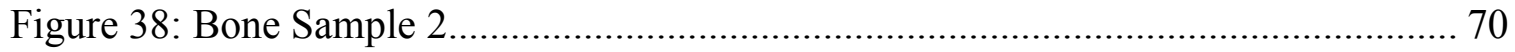

Figure 39: Side of bone sample 2 facing middle …............................................... 70

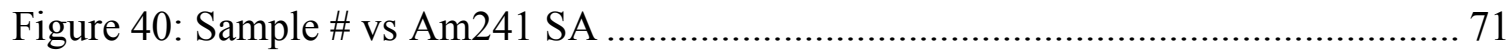




\section{List of Appendices}

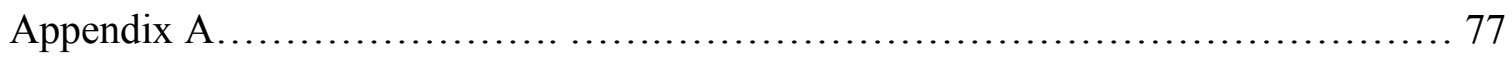

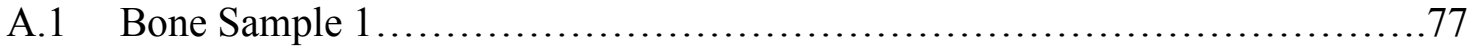

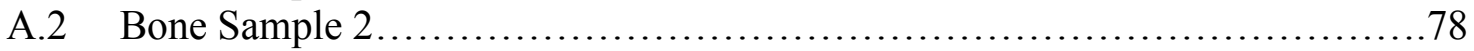




\section{List of Abbreviations}

DLLME - Dispersive Liquid-Liquid Micro Extraction

DTPA - DiethyleneTriaminePentaacetic Acid

EDTA- EthyleneDiamineTetraacetic Acid

FWHM - Full Width at Half Maximum

HDEHP - Di(2-ethylhexyl) phosphoric acid

HF-LPME - Hollow Fibre Liquid Phase Micro Extraction

HPLC-DAD - High-Performance Liquid Chromatography-Diode Array Detector

ICP-MS - Inductively Coupled Plasma Mass Spectrometry

ICP-OES - Inductively Coupled Plasma Optical Emission Spectrometry

ICRP - International Commission on Radiological Protection

LLE - Liquid Liquid Extraction

LSC - Liquid Scintillation Counting

MDA - Minimum Detectable Activity

PIPS - Passivated Implanted Planar Silicon

PTH - Parathyroid Hormone

$\mathrm{RN}$ - Radionuclear

ROI - Region Of Interest

RTG -Radioisotope Thermal Generator

SDME - Single Drop Micro Extraction

tSIE - transformed Spectral Index of External standard

USTUR - United States Transuranium Registry 


\section{Introduction}

Radiation protection is an area of science that emerged since the 1940's in response to the growing number of nuclear power plants and nuclear weapon tests. Over the years, power plant disasters such as the Chernobyl accident in 1986 and the Fukushima accident in 2011 have prompted the need for rapid tools to assess radionuclide contamination and also long-term studies on radiation in the human body.

Of particular concern are the long-lived transuranic actinides produced in the nuclear process, such as plutonium and americium, in the human body. If a person is exposed to these actinides, either through inhalation, ingestion, or absorption through wounds, these actinides will accumulate in organs such as the bone and continue to irradiate the surrounding tissues.

One area of radiation protection is to determine if a person has been internally exposed to radionuclides and to assess whether medical treatment is required. Some individuals who have been exposed will need continuous biomonitoring to ascertain the level of exposure and necessary treatment dosage/duration.

On the other extreme, radiation protection also focuses on long-term monitoring of exposed individuals and post-mortem tissue examinations. Data collected from these measurements serve to validate or refine existing dose models so that long-term risk can be better understood and thresholds for early intervention can be more accurately set.

This thesis focuses on the sample preparation, separation and measurement of actinides in biological samples using modern techniques. Part A will discuss the development of a method for screening plutonium in urine samples following an emergency, which is based on dispersive liquid-liquid extraction (DLLME). Part B will 
discuss the determination of americium in a bone sample taken from a deceased worker, which is based on solid phase extraction (SPE) and alpha spectroscopy, to investigate the distribution of americium for purposes of dose reconstruction. 


\section{Part A: Determination of Plutonium 238 in Urine Samples by Dispersive Liquid-Liquid Microextraction and Liquid Scintillation Counting or Alpha Spectroscopy}

\section{Background}

In a radiological or nuclear $(\mathrm{RN})$ emergency, first responders and the public face danger of internal contamination by radionuclides through inhalation, ingestion, or wounding. Rapid radiobioassay methods that deliver the timely assessment of internal contamination are important for identifying contaminated individuals for early medical intervention. Even when chelation therapy is provided to exposed individuals, radiobioassay can be used continuously to estimate the radiation dose received.

The most common biological sample used for internal radiation dose assessment is urine. As the radionuclides in urine are often present in trace quantities, there is a need to isolate them from the urine matrix before measurement using an appropriate instrument.

Traditional liquid-liquid extraction (LLE) involves the addition of an immiscible organic solvent to an aqueous sample containing the analyte of interest. The sample is then mixed, and the analyte would partition between the two phases depending on factors such as $\mathrm{pH}$, salt content, and polarity of the analyte. It requires a large amount of solvents and multiple re-extractions for high recovery and is time-consuming.

In the 1990s, many techniques focused on the miniaturization or increasing the preconcentration ability of traditional LLE were created. One of the earlier developments was single drop microextraction (SDME) where a single drop of water-immiscible extractant is suspended in a continuously stirred sample by a syringe, and the analyte is allowed to partition from the bulk solution into the droplet. After some time, the droplet 
is collected back into the syringe and analyzed. The drawback of this method is the possibility of the droplet of extractant to break off the syringe under fast stirring or in the presence of air bubbles [1]. This drawback was later solved by using hollow fiber liquid phase microextraction (HF-LPME), which works by a similar principle as SDME but replaces the organic droplet with a hydrophobic polymeric fiber containing an immobilized extractant [2]. This process allows for increased stir rates and applies to samples containing dissolved solids or complicated matrices. Disadvantages include possible contamination when handling the fiber and a reliance on hollow fiber manufacturers [3].

In 2006, dispersive liquid-liquid microextraction (DLLME) as an analytical technique was developed to be simple, fast, and reliant only on a small volume of organic solvents. The original variant of DLLME works by mixing a small volume of a high density (usually chlorinated) water-immiscible extraction solvent with a larger volume of a disperser solvent, and then rapidly injecting this extraction mixture into an aqueous sample. The high turbulence causes the formation of many small droplets and a large total surface area for the interaction of the aqueous analyte with the extractant solvent. This results in a fast equilibrium and short extraction times. Phase separation is done by centrifugation, and the organic fraction is analyzed [4]

Over the last nine years, DLLME as an effective separation and preconcentration method for complex matrices has been developed for dozens of applications. For the extraction of uranium, Chandrasekaran et al. [5] used ammonium pyrrolidine dithiocarbamate with cetyltrimethyl ammonium bromide to complex uranium and 
preconcentrate uranium from groundwater, with ICP-OES and ICP-MS for detection. Khajel et al. [6] also investigated the extraction of uranium in water using 1-(2pyridylazo)-2-naphthol as the extractant with detection by HPLC-DAD. Sadi et al. [7] developed a method to analyze Ra-226 in drinking water samples with detection by LSC, using a de-emulsifier for phase separation instead of centrifugation.

In all forms of liquid-liquid extraction, a common metric used to evaluate performance is the distribution ratio, which is simply the ratio of the analyte's concentration in the organic phase to that in the aqueous phase. An alternate representation used during method development is the \% recovery of the analyte into the organic phase (Equation 1, 2).

\section{Equation 1}

$$
D=\frac{[\text { analyte }]_{\text {org }}}{[\text { analyte }]_{\text {aq }}}
$$

Equation 2

$$
\% \quad \text { Recovery }=100 \times \frac{[\text { analyte }]}{[\text { expected }]}
$$

\section{$\underline{1.1 \text { Chemistry of Plutonium }}$}

Plutonium belongs to the actinide group of elements, and all of its isotopes are radioactive. Although there are trace levels of naturally occurring plutonium, the majority is produced during nuclear processes. Two plutonium isotopes, 238 and 239, are

particularly noteworthy because they emit high energy alphas $\left(\mathrm{He}^{2+}\right)$ and have long half- 
lives (the time required for an isotope to decay to half of its initial activity). Table 1 details the emission modes and decay energies of the plutonium isotopes along isotopes of americium and uranium, which are commonly present with plutonium in spent fuel. The tracer isotopes are also artificially produced and not present in spent fuel in high quantities, which make them ideal for use as internal standards during chemical separations. Equation (3) shows the synthesis route of $\mathrm{Pu}-238$ where U-238 is bombarded with deuterium to form $\mathrm{Np}-238$, which in turn decays by beta to $\mathrm{Pu}-238$ [8]. In equation 4, U-238 is first bombarded by neutrons to form U-239, which beta decays to Np-239 and further decays to $\mathrm{Pu}-239$.

\section{Equation 3:}

$$
\begin{aligned}
& { }_{92}^{238} \mathrm{U}+{ }_{1}^{2} \mathrm{H} \rightarrow{ }_{93}^{238} \mathrm{~Np}+2{ }_{0}^{1} \mathrm{n} \\
& { }_{93}^{238} \mathrm{~Np} \underset{2.1 \mathrm{~d}}{\stackrel{\beta^{-}}{\longrightarrow}}{ }_{94}^{238} \mathrm{Pu}\left(t_{1 / 2}=87.7 \text { years }\right)
\end{aligned}
$$

\section{Equation 4:}

$$
\begin{aligned}
& { }_{92}^{238} \mathrm{U}+{ }_{0}^{1} \mathrm{n} \rightarrow{ }_{92}^{239} \mathrm{U}+\gamma \\
& { }_{92}^{239} \mathrm{U} \underset{23.5 \mathrm{~min}}{\stackrel{\beta^{-}}{\longrightarrow}}{ }_{93}^{239} \mathrm{~Np} \underset{2.36 \text { days }}{\stackrel{\beta^{-}}{\longrightarrow}}{ }_{94}^{239} \mathrm{Pu}\left(t_{1 / 2}=24,110 \text { years }\right)
\end{aligned}
$$

Table 1: Actinides and their alpha decay energies

\begin{tabular}{llll}
\hline Isotope & Half Life $(\mathbf{y})$ & Decay modes & $\begin{array}{l}\text { Alpha energies } \\
\text { (keV) }\end{array}$ \\
\hline $\mathrm{Pu}-238$ & 87.7 & $\alpha$ & $5499(70.9 \%)$ \\
$\mathrm{Pu}-239$ & \multirow{2}{*}{24110} & $\alpha, \gamma$ & $5456(29.0 \%)$ \\
& & & $5157(70.8 \%)$ \\
$\mathrm{Pu}-240$ & 6563 & $\alpha$ & $5144(11.9 \%)$ \\
\hline
\end{tabular}




\begin{tabular}{|c|c|c|c|}
\hline $\mathrm{Pu}-241$ & 14.29 & $\beta$ & \\
\hline Am-241 & 432.2 & $\alpha, \gamma$ & $\begin{array}{l}5486(84.8 \%) \\
5443(13.1 \%)\end{array}$ \\
\hline U-235 & $703,800,000$ & $\alpha, \gamma$ & $\begin{array}{l}4395(57.7 \%) \\
4364(18.9 \%)\end{array}$ \\
\hline \multicolumn{4}{|l|}{ Tracers } \\
\hline $\mathrm{Pu}-236$ & 2.86 & $\alpha$ & $\begin{array}{l}5768(69.1 \%) \\
5721(30.8 \%)\end{array}$ \\
\hline $\mathrm{Pu}-242$ & 373000 & $\alpha$ & $\begin{array}{l}4902(76.5 \%) \\
4858(23.4 \%)\end{array}$ \\
\hline Am-243 & 7370 & $\alpha$ & $\begin{array}{l}5275(86.7 \%) \\
5233(11.5 \%)\end{array}$ \\
\hline U-233 & 159200 & $\alpha$ & $\begin{array}{l}4824(84.3 \%) \\
4784(13.2 \%)\end{array}$ \\
\hline
\end{tabular}

Plutonium has a ground state electron configuration of [Rn] 7s2 5f6. Compared to the lanthanides which " +3 " is the most common and stable oxidation state, plutonium and the other actinides have oxidation states that range from +3 to +6 . This is due to their $5 \mathrm{f}$ and $6 \mathrm{~d}$ orbitals being close in energy causing oxidating and reducing agents to have a greater influence on the valence state. The most common oxidation states for $\mathrm{Pu}$ are +4 and +3 , with the higher oxidation states forming $\mathrm{PuO}^{2+}$ and $\mathrm{PuO}_{2}{ }^{2+}[9]$

The separation of plutonium from other elements is often dependent on maintaining a constant valence for plutonium. Common reducing agents for $\mathrm{Pu}$ include ascorbic acid, sulfamic acid, and titanium chloride and common oxidizing agents for $\mathrm{Pu}$ include nitrite, and $\mathrm{HNO}_{3}$ (Equation 5) [10]. $\mathrm{HNO}_{3}$ is a common complexing acid used in nuclear fuel reprocessing because it forms many stable acid complexes with $\mathrm{Pu}^{4+}$.

\section{Equation 5:}

$3 \mathrm{Pu}^{3+}+\mathrm{NO}_{3}^{-}+4 \mathrm{H}^{+}->3 \mathrm{Pu}^{4+}+\mathrm{NO}+2 \mathrm{H} 2 \mathrm{O}$ 


\section{$\underline{1.2}$ Concerns regarding $\mathrm{Pu}-238$}

$\mathrm{Pu}-238,239$, and 240 are all nuclear activation products and are found as mixtures of spent nuclear fuel, and in the environment as a result of nuclear weapon testing or accidental release from nuclear facilities. $\mathrm{Pu}-238$ is used in radioisotope thermoelectric generators (RTGs) because of its shorter half-life and high thermal yield. Since the 1950's, RTGs have been used by the United States and the Soviet Union to power satellites and spacecraft, such as the Voyager. The Soviet Union also used RTGs for lighthouses, communication towers, and other remote sites where access to electricity was limited. [11] Accidental disintegration of satellites when they fall out of orbit have also released 0.63 PBq of $\mathrm{Pu}-238$ into the atmosphere [12].

Many of these RTGs have been abandoned or orphaned after the dismantling of the Soviet Union. There is a risk that these sources are recovered and used as radiological weapons. One of the radiological weapons of concern is the dirty bomb, which goal is to contaminate a populated area with high radioactivity using conventional explosives as the mechanism for dispersion [13].

\section{$\underline{1.3 \text { Extractants }}$}

Potential extractants for plutonium are characterized based on the mechanism of interaction. Neutral organic extractants have electron donor groups which can form ion association complexes with actinides. Acidic extractants rely on cation exchange and 
hydrogen is replaced with the actinide cation. Amine extractants are long chain alkyl cations which bind to actinide-anion complexes.[14]

The alkyl phosphoric acids were initially developed for uranium extraction [15]. However, studies in the past have used the acidic extractant, bis(2-ethylhexyl) phosphate (HDEHP), to extract plutonium from highly acidic chloride media [16] and clay samples [17]. Figure 1 shows the chemical structure of HDEHP and Equation 6 shows the formation of a dimeric HDEHP-metal complex [18].

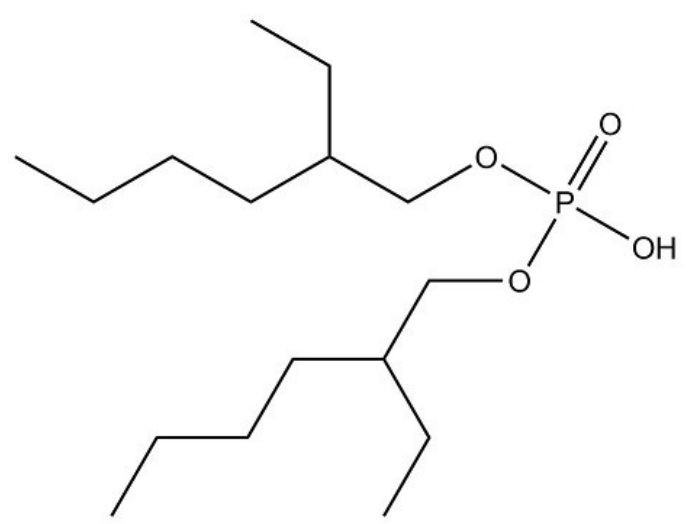

Figure 1: Structure of HDEHP.

\section{Equation 6:}

$$
\mathrm{M}^{\mathrm{n}+}+\mathrm{n}(\mathrm{HA})_{2}(\text { org }) \longleftrightarrow \mathrm{M}\left(\mathrm{HA}_{2}\right)^{\mathrm{n}}(\text { org })+\mathrm{nH}^{+}
$$

\subsection{Sample pretreatment}

Typical DLLME procedures work with small volumes of samples $(<10 \mathrm{ml})$ for even dispersion of the extractant mixture within the total sample volume and for limiting the amount of extractant and solvent required. Often, the amount of plutonium in a typical $10 \mathrm{ml}$ urine sample is not enough to be accurately determined using currently available technologies. In these cases, sample pretreatment procedures can be used to preconcentrate $\mathrm{Pu}$ from larger volumes. For emergency urine bioassay samples (< 
$100 \mathrm{ml}$ ), direct evaporation and acid digestion can be used. For urine samples between $100 \mathrm{ml}$ and $1 \mathrm{~L}$, co-precipitation of $\mathrm{Pu}$ with other insoluble compounds, such as $\mathrm{Ca}_{3}\left(\mathrm{PO}_{4}\right)_{2}$ [19], $\mathrm{Fe}(\mathrm{OH})_{3}$ [20] and $\mathrm{HTiO}$ [21], and followed by acid digestion, have been reported in the literature.

\section{$\underline{1.5 \text { Speciation consideration }}$}

In cases where an individual is known to be exposed to plutonium, medical intervention may begin immediately following gross alpha/beta screening of urine, skin swipes, or nasal swabs. The most common treatment for transuranics is chelation therapy with a strong actinide complexing agent such as diethylenetriaminepentaacetic acid (DTPA). The stability of Pu-DTPA is very high and this complex remains intact through the bioelimination process. This means subsequent measurements of $\mathrm{Pu}$ in urine will require decomposition of this complex as it will prevent extraction of $\mathrm{Pu}$ by organic ligands [22].

Previous studies on nuclear reactor effluents or environmental water samples use a variety of oxidation methods to decompose DTPA or similar complexing agents such as EDTA and NTA, by $\mathrm{KMnO}_{4}$ or $\mathrm{H}_{2} \mathrm{O}_{2}[23,24]$.

\subsection{Detection methods for radiation}

Based on the mode of radioactive decay, detection methods have been developed to measure the radiation directly in the case of alpha and gamma spectroscopy or through secondary photon emissions as in the case of liquid scintillation counting. For longerlived radionuclides, inductively coupled plasma mass spectrometry has been used in the past two decades to look for isotopes based on their mass concentration. The choice of 
detector depends on the sensitivity required, the presence of interfering radionuclides and the degree of resolution required. For example, when the pure alpha emitter, $\mathrm{Pu}-238$ is present in a radionuclide mixture, requires chemical separation from other alphas with the same decay energy and detection with alpha spectroscopy is required.

\subsubsection{Liquid scintillation counting}

In liquid scintillation counting (LSC), the aqueous or organic liquid is mixed directly with a scintillation cocktail containing aromatic solvents, light emitting fluors, and emulsifiers. For the measurement of $\mathrm{Pu}-238$, the kinetic energy of the alpha particle emitted from $\mathrm{Pu}-238$ is transferred to the pi-electrons of the solvent and then to the scintillator or fluor. When the fluor relaxes to the ground state, fluorescence is emitted and the amount of fluorescence produced is directly proportional to the number of alpha decays in the sample vial. A photomultiplier tube amplifies and converts the photons to electrons and multiplies the signal into a pulse that is recorded as a count.

Many factors can influence the suppression or quenching of the fluorescent signal. Color quenching occurs when light is attenuated by colored compounds. $\mathrm{HNO}_{3}$ and $\mathrm{HCl}$ are both examples of chemical quenchers because they can capture electrons transferred between solvents and fluor, reducing the intensity of the signal or even terminating it $[25]$.

One way to assess the amount of quenching is by using the transformed external standard spectrum (tSIE) quench parameter. An external Ba133 gamma source is used to irradiate the scintillator and Compton scattering occurs to produce a lower energy gamma and a resultant Compton electron. This Compton electron behaves similarly to a beta 
particle and produces a Compton spectrum. Because it is within the LSC vial, all effects of the sample including quench will affect it. The LSC then uses algorithms such as tSIE to transform the Compton spectrum so a dependency on the endpoint of the spectrum versus the amount of quench in the sample can be achieved.

Most liquid scintillation counters use a 4-pi geometry that surrounds both sides of the sample with a PMT and allows close to $100 \%$ counting efficiency. Sometimes, there is background or luminescent events that can cause a signal at the PMT, and if they are not produced at the same time for PMT 1 and 2, they are gated from further signal amplification. Photons from the sample that do coincide are summed and amplified in the instrument's electronics [26]

\subsubsection{Alpha spectrometry}

Modern alpha spectrometers require passivated ion-implanted planar silicon (PIPS) detectors which have passivated $\mathrm{SiO}_{2}$ junctions capable of high alpha resolution (FWHM of $30 \mathrm{keV}$ ) and detector stability. These detectors work under the same principle as $\mathrm{p}+\mathrm{n}$ type semiconductor detectors. Solid samples are placed under the detector (cathode) in a vacuum chamber and a reverse bias is applied to create an electric field. Under this condition and in the absence of ionizing radiation, there is no current flow. When there are alpha emitters present in the sample, they can create free electrons and holes within the detector material and some of these electrons are promoted from the valence band to the conduction band and carried to the detector electronics. The number 
of these electron-hole pairs produced is proportional to the energy of the emitter and the frequency of these pair formations is proportional to the activity of the emitter [27].

There are a number of factors in detector design and sample preparation that can influence the resolution or efficiency. For high resolution, a thin entrance window prevents energy straggling or alpha particles being attenuated before they hit the detector. This keeps a narrow energy spread for the alpha particles and a sharper peak. A low leakage current keeps electronic noise to a minimal because having low or zero backgrounds is often crucial to low-level detection. By decreasing the distance between detector and source, a better alpha efficiency is achieved but higher energy straggling is observed and the FWHM increases. As the radius of the sample source increases, the efficiency decreases because there is a higher chance the alpha decay particles do not reach the detector [15].

Since the alpha particles interact with the detector directly, very clean samples are required. One method is direct evaporation of active solutions onto a disk but this is often inconsistent and matrix components can attenuate the signal. Electrodeposition of the radioisotope onto stainless steel allows for a source with high resolution because there are no interfering components but the apparatus for electrodeposition requires constant monitoring and it can take up to 1-2 hours to complete.

Another method is using microprecipitation, where actinides are coprecipitated along with a lanthanide fluoride which forms very fine precipitates. For example, when cerium nitrate is added to a solution containing $\mathrm{Pu}^{3+}, \mathrm{Am}^{3+}$, and $\mathrm{HF}$, cerium fluoride precipitate is formed and plutonium and americium are scavenged along with it. It is 
preferable to form this thin uniform layer for the detection of alpha particles because otherwise, alpha emissions can be "self-absorbed" within the carrier material itself [29].

\subsection{Scope of study}

1) To develop a dispersive liquid-liquid microextraction method for plutonium, with known isotopic composition, in urine samples for emergency screening

2) To adapt this method to samples containing isotopic plutonium, complexed plutonium, multiple actinides, or a large volume.

\section{$\underline{\text { Experimental }}$}

\subsection{Materials and apparatus}

\subsubsection{Reagents}

All dilute solutions were prepared using distilled water filtered through aMillipore filtration system $(16 \mathrm{M} \Omega / \mathrm{cm})$. Concentrated nitric, hydrochloric acid, ammonium hydroxide, and hydrogen peroxide were of ACS grade (Fisher Scientific, Canada). Bis(2ethylhexyl) phosphate, chloroform, dichloromethane, tetrachloroethylene were of ACS grade (Sigma-Aldrich, Canada). Ascorbic acid, cerium nitrate, hydrofluoric acid, and sodium nitrite were of trace metal grade (Sigma-Aldrich, Canada). Calcium nitrate, sodium oxalate, sodium bicarbonate and potassium persulfate were of ACS grade (Fisher Scientific, Canada). Hydrazine (30\%) was purchased from Sigma Aldrich (Canada). Filter discs and funnels were purchased from Eichrom (USA) and mounting adhesives were purchased from Soquelec (Canada). Liquid scintillators were purchased from Perkin-Elmer (USA).

\subsubsection{Isotopes}


All isotopes used for sample preparation were NIST-certified and purchased from Eckert and Ziegler (USA), with the exception of Am-241 which was purchased from Amersham (UK).

All intermediate isotope solutions used for spiking were diluted with $0.5 \mathrm{M} \mathrm{HNO}_{3}$ and the volume added to each sample ranged from $50 \mathrm{uL}$ to $200 \mathrm{uL}$. This was done to prevent the spiking solution from changing the working $\mathrm{pH}$ of the sample. For tests with Pu-DTPA, the plutonium intermediate solution was equilibrated with Na-DTPA for 4 hours to achieve a final DTPA concentration of $0.02 \mathrm{M}$. Since all isotopes of plutonium have the same chemical properties, $\mathrm{Pu}-239$ and $\mathrm{Pu}-238$ were used interchangeably for method development with LSC. For processes containing both plutonium and americium and measurement by alpha spectroscopy, Pu-239 was used to ensure no overlap with the Am-241 signal when calculating recoveries.

At Health Canada's Radiation Protection Bureau, the National Calibration Reference Centre collects urine from healthy donors. This urine is pooled and preserved in $1 \% \mathrm{HCl}$ before spiking with different isotopes and sent to client laboratories to validate if their methods meet Canadian Nuclear Safety Commission (CNSC) requirements. Often, there is extra unspiked urine remaining from these intercomparison programs that are available for use.

\section{$\underline{\text { 2.2 Methods }}$}

\subsubsection{DLLME procedure}

For method development purposes, all extractions, unless stated otherwise, were performed on $10 \mathrm{~mL}$ of urine in $15 \mathrm{~mL}$ centrifuge tubes using approximately $2 \mathrm{~mL}$ of 
extraction mixture. This was done to conserve reagents due to a large number of experiments and replicate samples. The extraction mixture at the very start was $1.5 \mathrm{~mL}$ $\mathrm{MeOH}, 100 \mathrm{uL} \mathrm{CHCl}_{3}$, and $100 \mathrm{uL}$ of HDEHP. These volumes were selected based on an average of literature values for disperser/extractant volumes ratios. After each sequential optimization experiment, these volumes were refined and $\mathrm{C}_{2} \mathrm{Cl}_{4}$ replaced $\mathrm{CHCl}_{3}$. The type of syringe used for all injections was the Hamilton (USA) Gastight fixed needle syringe with 0.22 gauge cone tip either in $5 \mathrm{~mL}$ or $10 \mathrm{~mL}$ capacities.

Samples containing $10 \mathrm{~mL}$ urine acidified to $1 \% \mathrm{HCl}(\mathrm{pH}=1-1.1)$ were acidified to $\mathrm{pH} 0.8$ using $0.2 \mathrm{~mL}$ of $8 \mathrm{M} \mathrm{HNO}_{3}$. $2-5 \mathrm{~Bq}$ of $\mathrm{Pu}-238$ or $\mathrm{Pu}-239$ were added. The extraction mixture with a total volume of $2.2 \mathrm{~mL}$ was rapidly injected into the sample using a $10 \mathrm{~mL}$ syringe. A cloudy dispersion was formed and this mixture was sonicated at room temperature for 5 minutes and then centrifuged at $3200 \mathrm{rcf}$ for an additional 5 minutes. A clear phase separation is formed after centrifugation, and the aqueous supernatant is removed. The centrifuge tube containing the sedimented pellet is then immersed in a $90^{\circ} \mathrm{C}$ water bath and $\mathrm{C}_{2} \mathrm{Cl}_{4}$ is evaporated under a stream of nitrogen. The pellet is then re-dissolved completely in $0.5 \mathrm{~mL}$ of toluene and $2 \times 1 \mathrm{~mL}$ of methanol and transferred to a plastic LSC vial. This was mixed with $17 \mathrm{~mL}$ of Hi-Ionic Fluor scintillation cocktail and equilibrated in the dark 1 hour before counting.

\section{$\underline{\text { 2.2.2 Back-extraction }}$}

For samples containing multiple isotopes or activities too low for liquid scintillation counting, alpha spectroscopy is required. In order to prepare the sources for alpha spectroscopy, plutonium or other actinides have to be first back-extracted from 
organic extractant into an aqueous phase. After the sedimented pellet is isolated following the first extraction, it is re-suspended in $0.5 \mathrm{~mL}$ of kerosene and $0.5 \mathrm{~mL}$ of $\mathrm{C}_{2} \mathrm{Cl}_{4}$ to give a dense organic phase. Then three washes of $3 \mathrm{~mL}$ of a back-extractant are added, with centrifugation and the removal of the aqueous phase after each wash. During method development when back extractants were tested on singular isotopes, the aqueous fraction was measured by LSC.

\section{$\underline{\text { 2.2.3 Alpha spectroscopy source preparation }}$}

When multiple isotopes are present, alpha source preparation based on coprecipitation of plutonium with lanthanide fluorides was used. The back extracted solutions are diluted to a volume of $10 \mathrm{~mL}$ using $0.5 \mathrm{M} \mathrm{HCl}$. Then, in the following order,

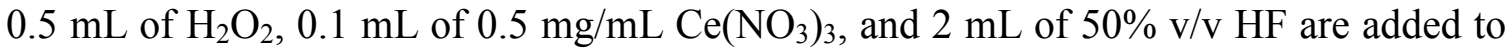
the sample. This mixture is sonicated for 15 minutes and rested for another 15 minutes. Then a disposable filter funnel fitted with a polypropylene filter disc (0.1um pore size, $25 \mathrm{~mm}$ dia.) is attached to a vacuum box and preconditioned with $2 \times 1.5 \mathrm{~mL}$ of $80 \%$ ethanol. Afterward, the sample is filtered through the funnel at $\sim 5$ torr, with constant rotation of the filter funnel to allow for consistent deposition on the filter disc. The sample is then rinsed with $80 \%$ ethanol, and dried under a heat lamp until visibly dry. The filter disc is then attached to an adhesive disk and tweezers were used to gently press down any air pockets that forms.

\section{$\underline{\text { 2.3 Instrumentation }}$}

Liquid scintillation counting was done using a Tri Carb 3180 TR/SL using 1800 3600s count times with tSIE as a quench indicating parameter. All samples were counted 
in $20 \mathrm{~mL}$ HDPE vials. The integration window was set to $60-440 \mathrm{keV}$ and all spectra were manually checked to see if the sample peak fell within this window. Alpha spectrometry counting was performed on an 8 chamber Ortec Octete fitted with $450 \mathrm{~mm}^{2}$ PIPS detectors and counted for 12 hours. Integration windows or ROIs were set to encompass entire alpha peaks with the exact channels varying between the 8 detectors.

\section{Results and Discussion}

\subsection{Choice and volume of disperser solvent}

The disperser should be miscible with the aqueous sample, the extraction solvent, and the extractant. Methanol, acetonitrile, acetone, and tetrahydrofuran were evaluated for their performance by mixing $1.5 \mathrm{~mL}$ of each individual solvent with $100 \mathrm{uL}$ HDEHP, and $100 \mathrm{uL} \mathrm{CHCl}_{3}$ (Table 2).

Table 2: Choice of disperser

\begin{tabular}{ll}
\hline Disperser Solvent & Mean \% Recovery $(\mathbf{n}=\mathbf{3})$ \\
\hline Methanol & $86.5 \pm 7.6$ \\
Acetonitrile & $78.2 \pm 2.7$ \\
Acetone & $73.3 \pm 5.3$ \\
Tetrahydrofuran & $62.4 \pm 8.7$ \\
\hline
\end{tabular}

Methanol had the highest recovery. This may be because methanol is a polar protic solvent, whereas the others are polar aprotic, and this allows it to have a higher compatibility and dispersibility within the aqueous phase. The optimal volume of methanol was tested by creating a series of extractant mixtures with the only varying quantity being the dispersing solvent's volume. A minimum volume of disperser solvent is required to effectively disperse the fine droplets of extraction solvent evenly 
throughout the sample. There was no apparent difference in recovery at volumes higher than $1.5 \mathrm{ml}$ and $2 \mathrm{ml}$ was chosen as the disperser solvent volume (Fig.2).

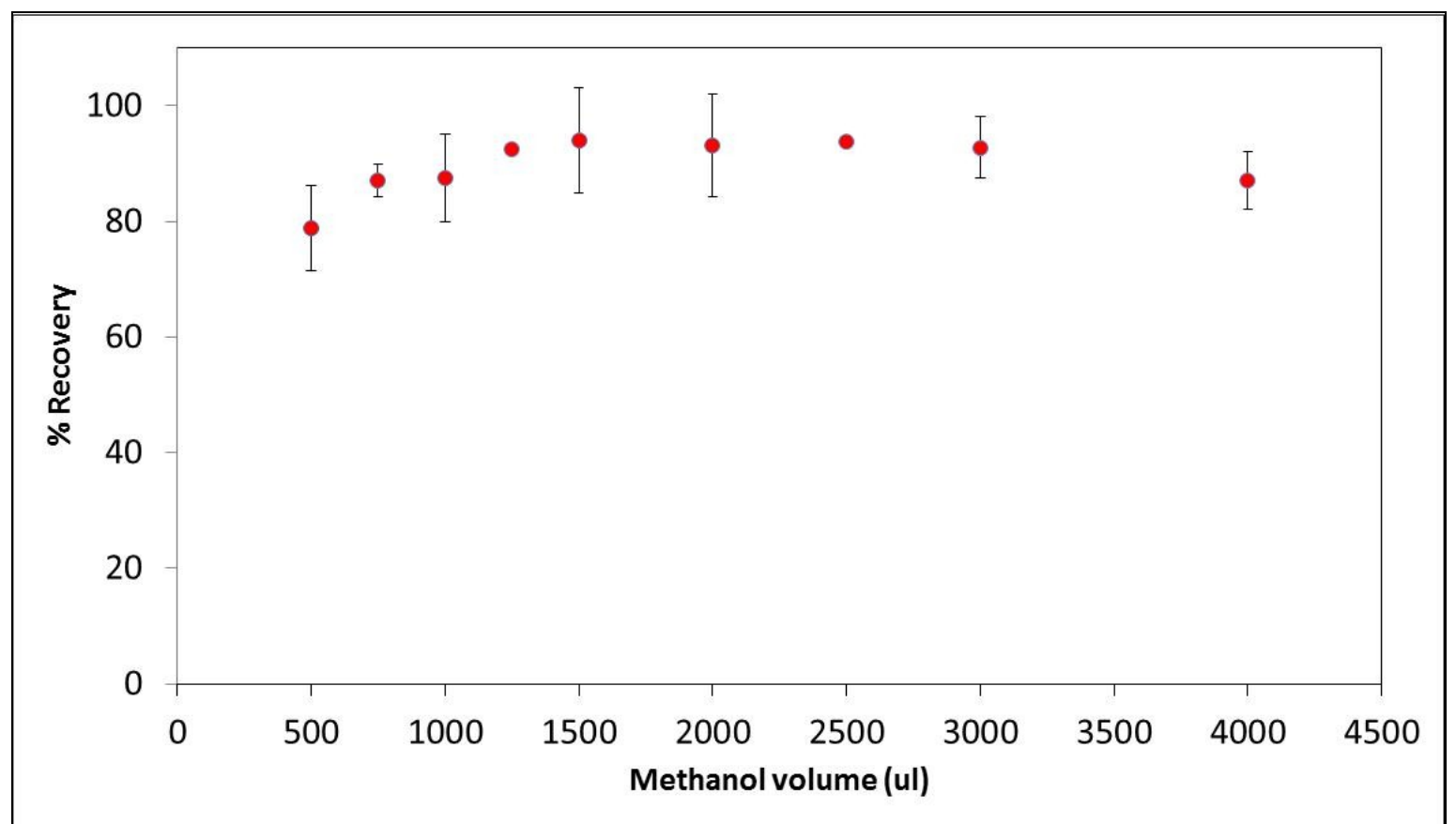

Figure 2: Effect of methanol volume on recovery.

\subsection{Choice and volume of extraction solvent}

The extraction solvent should be immiscible with the aqueous sample, and have a higher density to allow for separation by centrifugation. Dichloromethane, chloroform, and tetrachloroethylene were compared for their performance as extractant solvents and $100 \mathrm{uL}$ of each individual solvent was mixed with $100 \mathrm{uL}$ HDEHP, and $1.5 \mathrm{~mL}$ of $\mathrm{MeOH}$.

Dichloromethane was the worst of the three solvents and only 1 out of the 3 replicate samples formed a sedimented pellet. Out of the three solvents, it has the highest volatility and miscibility in water and also the lowest density. Chloroformed showed a recovery of $67 \pm 11 \%$ recovery $(n=7)$ and tetrachloroethylene showed a recovery of $78 \pm$ 
$7 \%(n=6)$. For dialkylphosphoric acid extractants, the extraction efficiency tends to decrease with an increase in the solvent's dielectric constant. This is attributed to increased hydrogen bonding of the solvent with the extractant [14].

To determine the optimal volume of extraction solvent, different extraction mixtures were created where the amount of $\mathrm{C}_{2} \mathrm{Cl}_{4}$ was varied (Fig.3). A volume of $25 \mathrm{uL}$ was chosen because there was little difference in recovery between $25-100 \mathrm{uL}$ of $\mathrm{C}_{2} \mathrm{Cl}_{4}$ and a smaller volume would result in a larger pre-concentration factor. Higher volumes showed a decrease in recovery, possibly from reduced dispersibility.

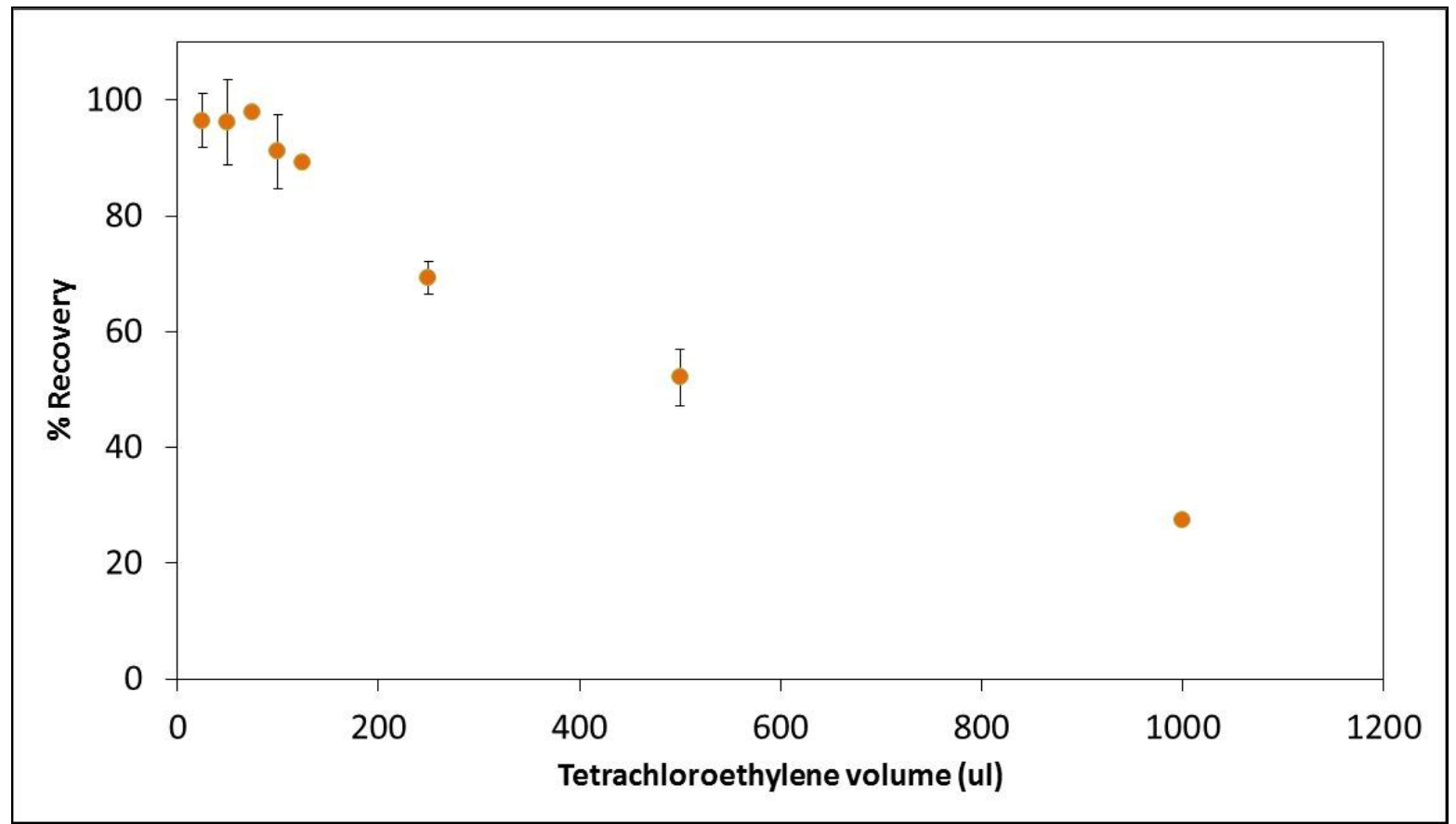

Figure 3: Effect of tetrachloroethylene on recovery.

\section{$\underline{3.3 \text { Volume of extractant }}$}

Lower volumes of the extractant between $25 \mathrm{uL}-100 \mathrm{uL}$ showed high variability in recovery. Between $100 \mathrm{uL}-200 \mathrm{uL}$, the recovery was at the highest and most reproducible. At volumes exceeding $500 \mathrm{uL}$, the combined organic phase of HDEHP and 
$\mathrm{C}_{2} \mathrm{Cl}_{4}$ is no longer denser than water and a sedimented pellet containing the HDEHP does not form after centrifugation (Fig. 4).

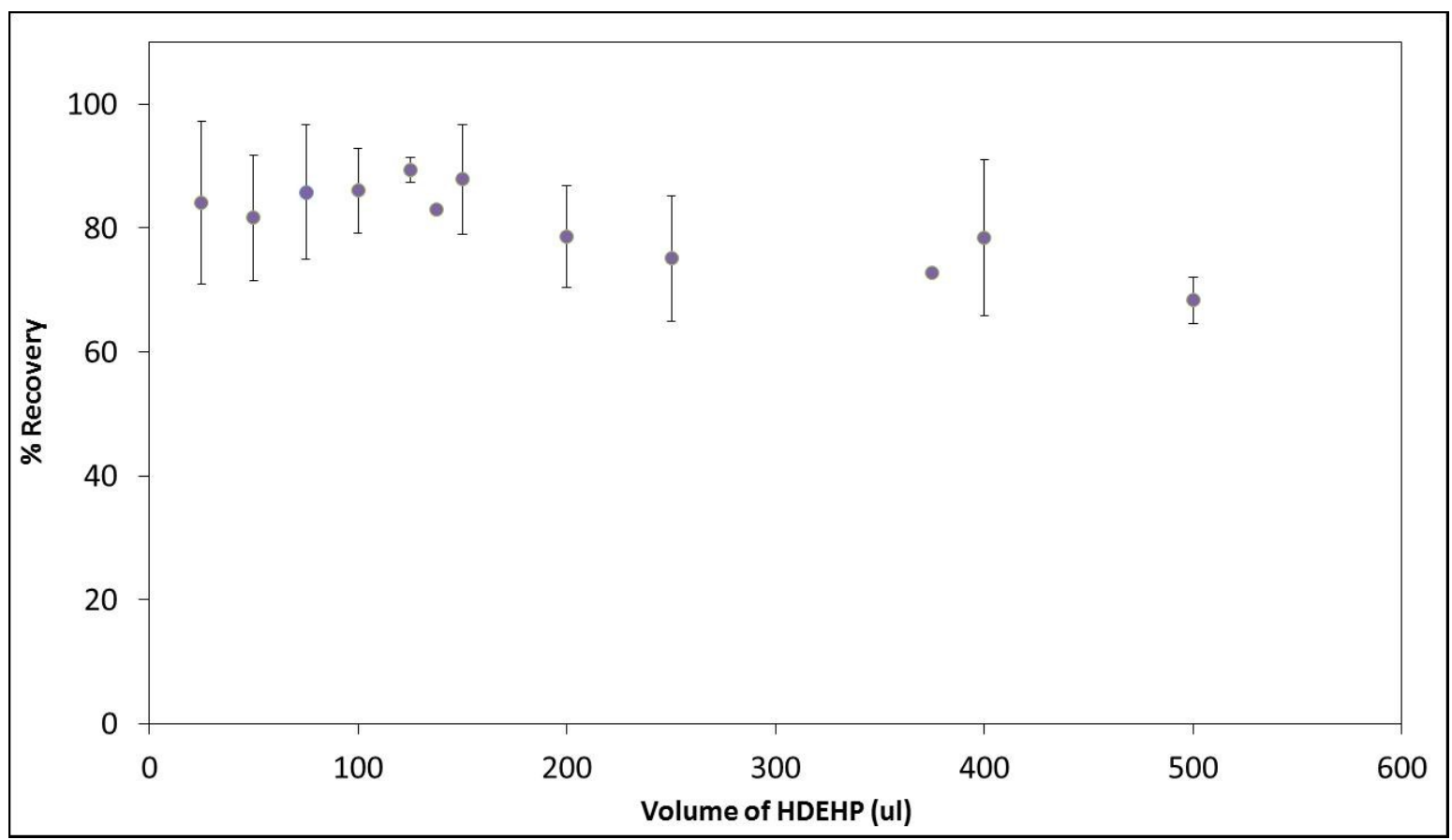

Figure 4: Effect of HDEHP volume on extraction.

\subsection{Effect of pH}

The $\mathrm{pH}$ of the pooled urine from the donors was first measured to be around 1.11.3 when acidified with $1 \% \mathrm{HCl}$ and the urine was evenly split into five or more equal fractions. Repeated trials of the $\mathrm{pH}$ curve added more points to regions where there was a maximum in recovery $(\mathrm{pH} 0.5-2)$ to better establish the trend in these regions. For $\mathrm{pH}$ points below the $1.1-1.3,8 \mathrm{M} \mathrm{HNO}_{3}$ was added dropwise and measured using a calibrated $\mathrm{pH}$ meter . For points above $\mathrm{pH} 1.1-1.3,1.5 \mathrm{M} \mathrm{NaOH}$ was used. A high molarity of acid or base was selected for these experiments to limit the change in sample volume. A similar methodology was used in the preparation of the $\mathrm{pH}$ curves for Am-241 and U-233. 
The extractability of plutonium by HDEHP in various acids and under many $\mathrm{pH}$ conditions has been well studied. High concentrations $(>0.1 \mathrm{M})$ of acids with complexing anions such as phosphoric or sulphuric acid causes reduced or no extraction of plutonium by HDEHP. Because of this, nitric and hydrochloric acid were chosen to acidify samples as they are weakly complexing and the highest reported recoveries for HDEHP extraction was done in those media.

The highest recovery was around $\mathrm{pH} 0.9$ and it is assumed that most plutonium is in the +4 state due to oxidation by $\mathrm{NO}_{3}{ }^{-}$and some plutonium has disproportionated to +3 or +6 . Between $\mathrm{pH} 1-4$, plutonium begins to hydrolyze to $\mathrm{Pu}(\mathrm{OH})^{3+}, \mathrm{Pu}(\mathrm{OH})_{2}{ }^{2+}$, $\mathrm{Pu}(\mathrm{OH})^{3+}$, and these complexes are less extractable by HDEHP. Above $\mathrm{pH} 4$, insoluble $\mathrm{Pu}(\mathrm{OH})_{4}$ forms and is unextractable by HDEHP which relies on cation exchange [30].

The high standard deviation for each is the result of performing the $\mathrm{pH}$ adjustment on different pooled urine samples. This was done to estimate a volume of $\mathrm{HNO}_{3}$ to add to a sample so that it will be in the $\mathrm{pH}$ range where high recovery is achieved. However, variation between batches can have recoveries that vary between $10-15 \%$ which is described more in section 3.15. Plutonium showed optimal recovery between $\mathrm{pH} 0.8-$ 1.1 (Fig. 5). The $\mathrm{pH}$ range for americium is comparable to that of plutonium's, but it drops off closer to zero recovery at low $\mathrm{pH}$ (Fig 6). This property prompts further investigation on potential back extraction conditions for americium from plutonium. In contrast, uranium shows very high recover even at the acidic pHs (Fig. 7). 


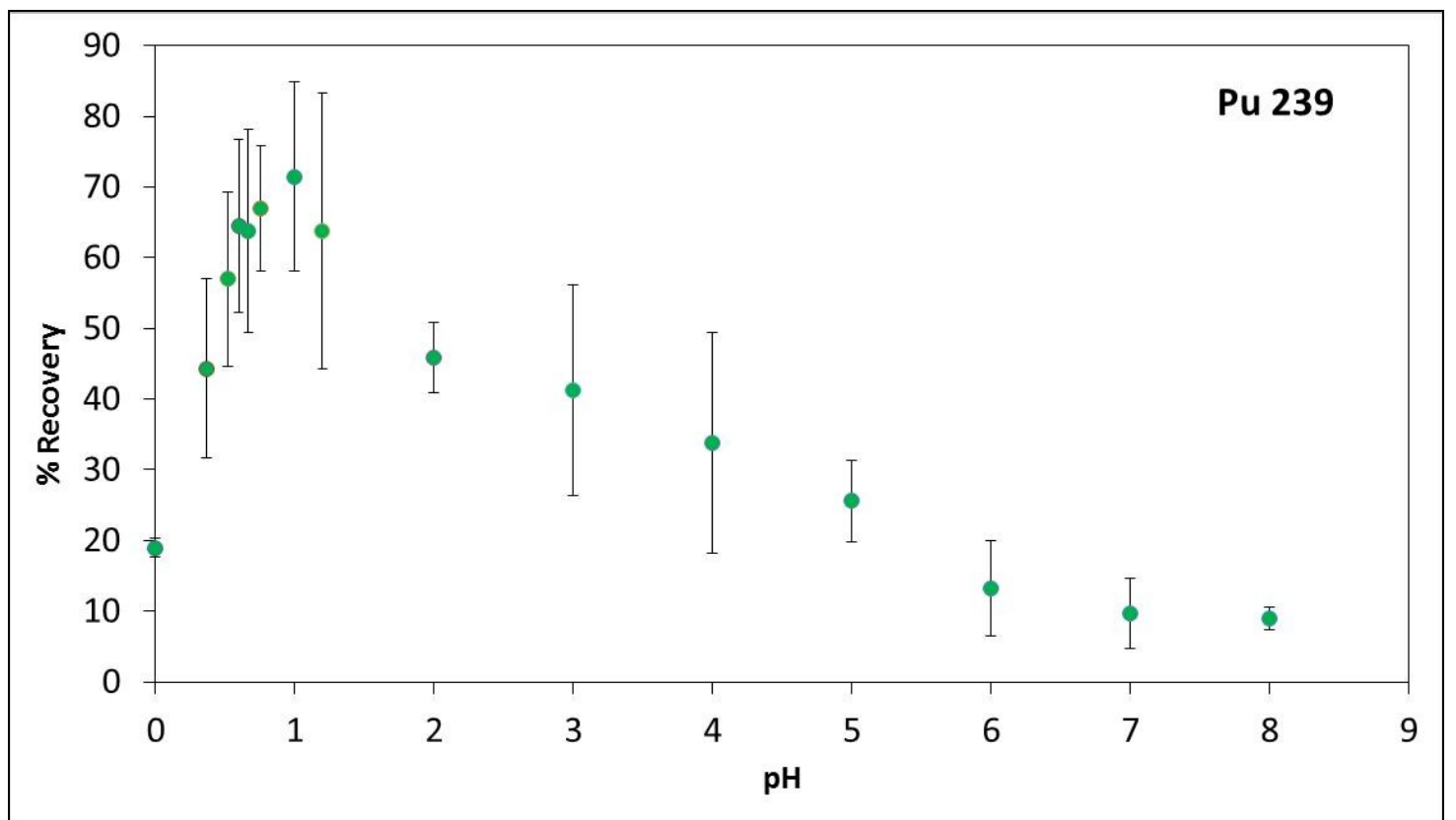

Figure 5: Effect of $\mathrm{pH}$ on recovery on plutonium

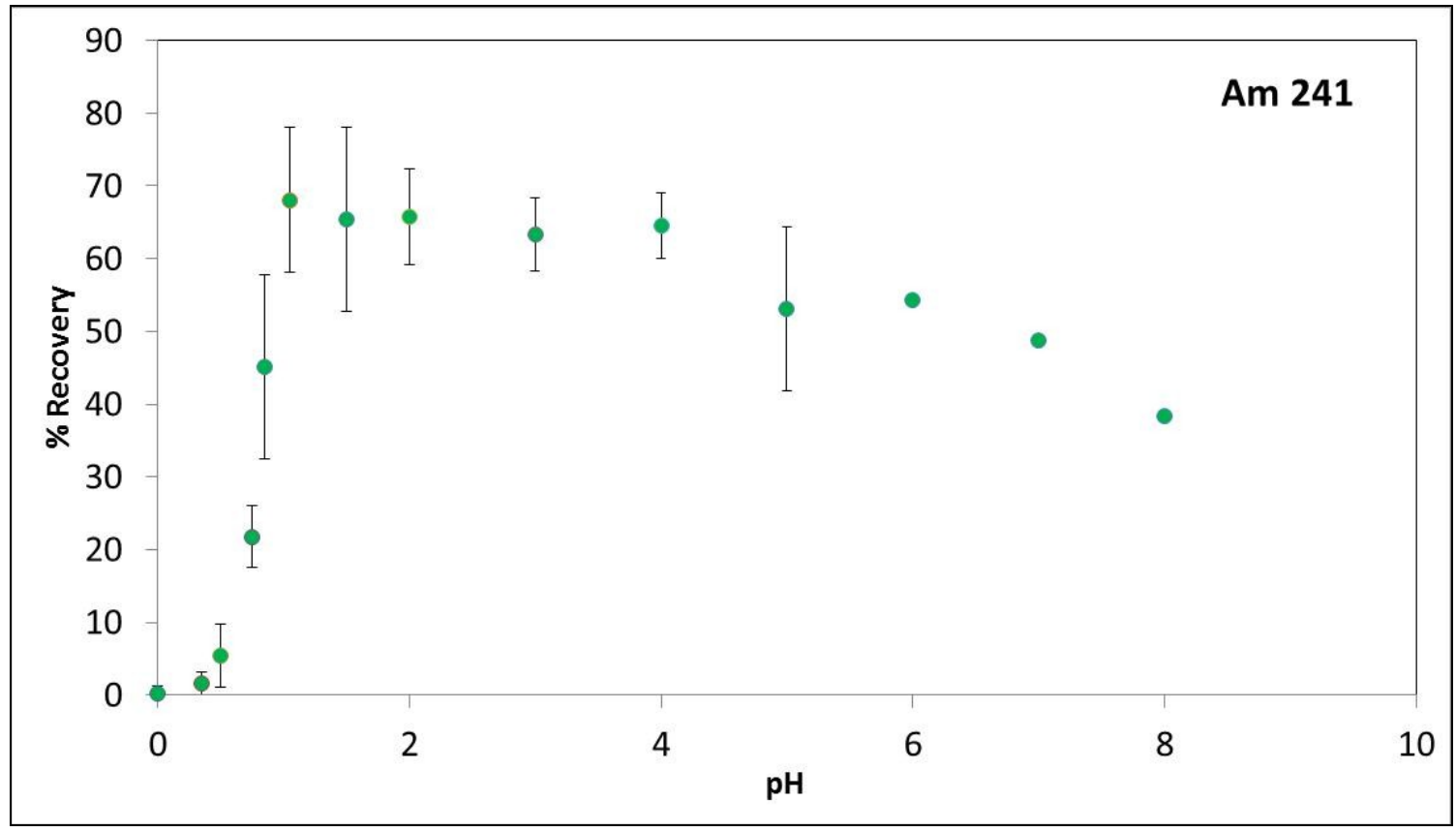

Figure 6: Effect of $\mathrm{pH}$ on recovery of americium 


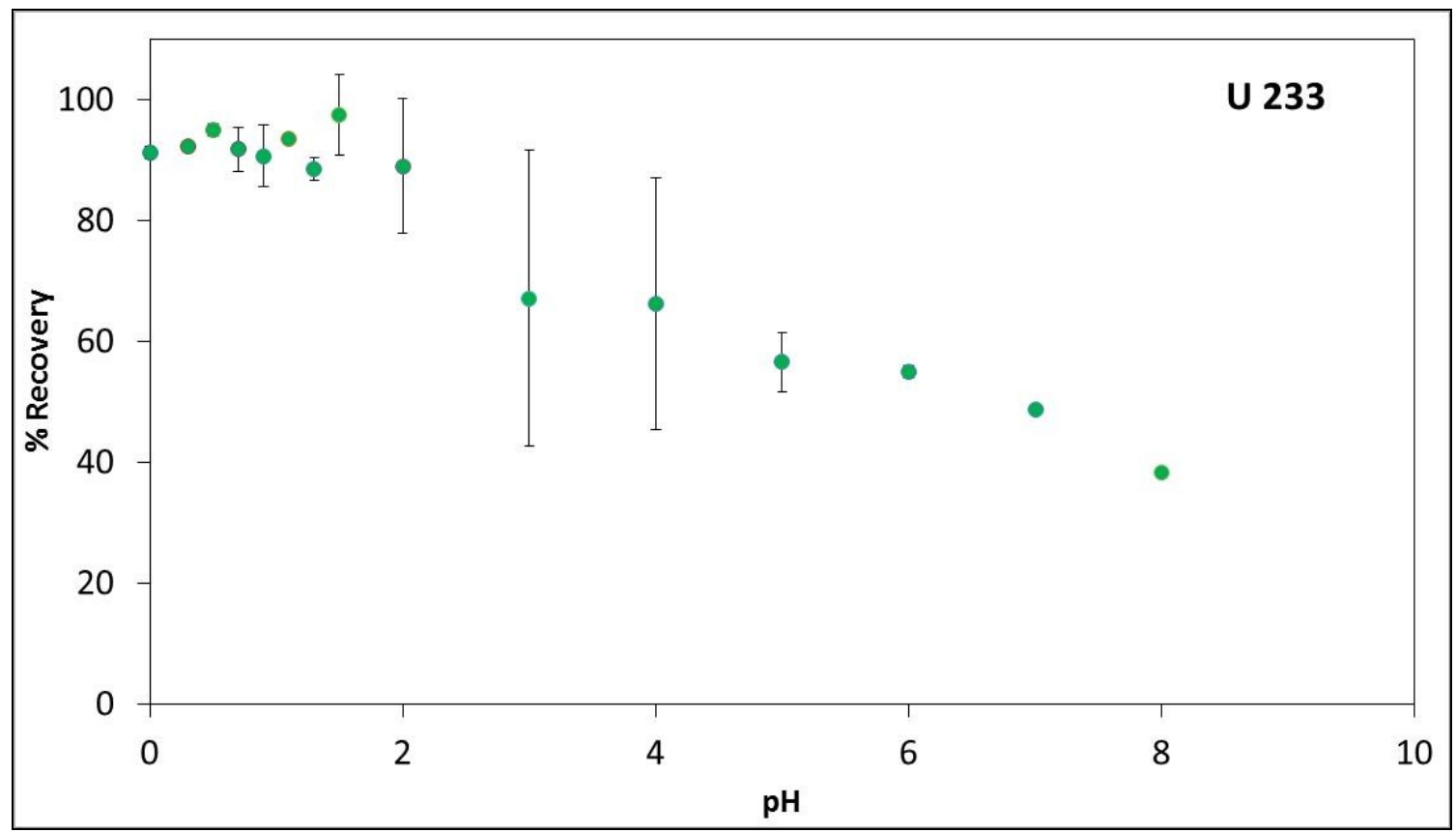

Figure 7: Effect of $\mathrm{pH}$ on recovery of uranium

\section{$\underline{3.5 \text { Effect of salt }}$}

The ionic strength of the sample was adjusted by adding $\mathrm{Ca}\left(\mathrm{NO}_{3}\right)_{2}$ (s) to the urine samples to achieve concentrations of $0.005 \mathrm{M}-0.2 \mathrm{M}$. Also, urine naturally contains high levels of salt, and the additional $\mathrm{Cl}^{-}$and $\mathrm{NO}_{3}{ }^{-}$ions from the acids also contribute to the ionic strength. In some DLLME processes for organic molecules that do not use a specific extractant within the extractant solvent, more polar analytes benefit from the salting out effect which favors the transfer of analytes into the organic phase. In the current case where HDEHP and Pu form a non-polar complex, the addition of salt is seen to cause a slight decrease in recovery (Fig. 8). This may be because the high ion content can mask the presence of plutonium at the interface of the extraction solvent droplet and the aqueous phase [31]. 


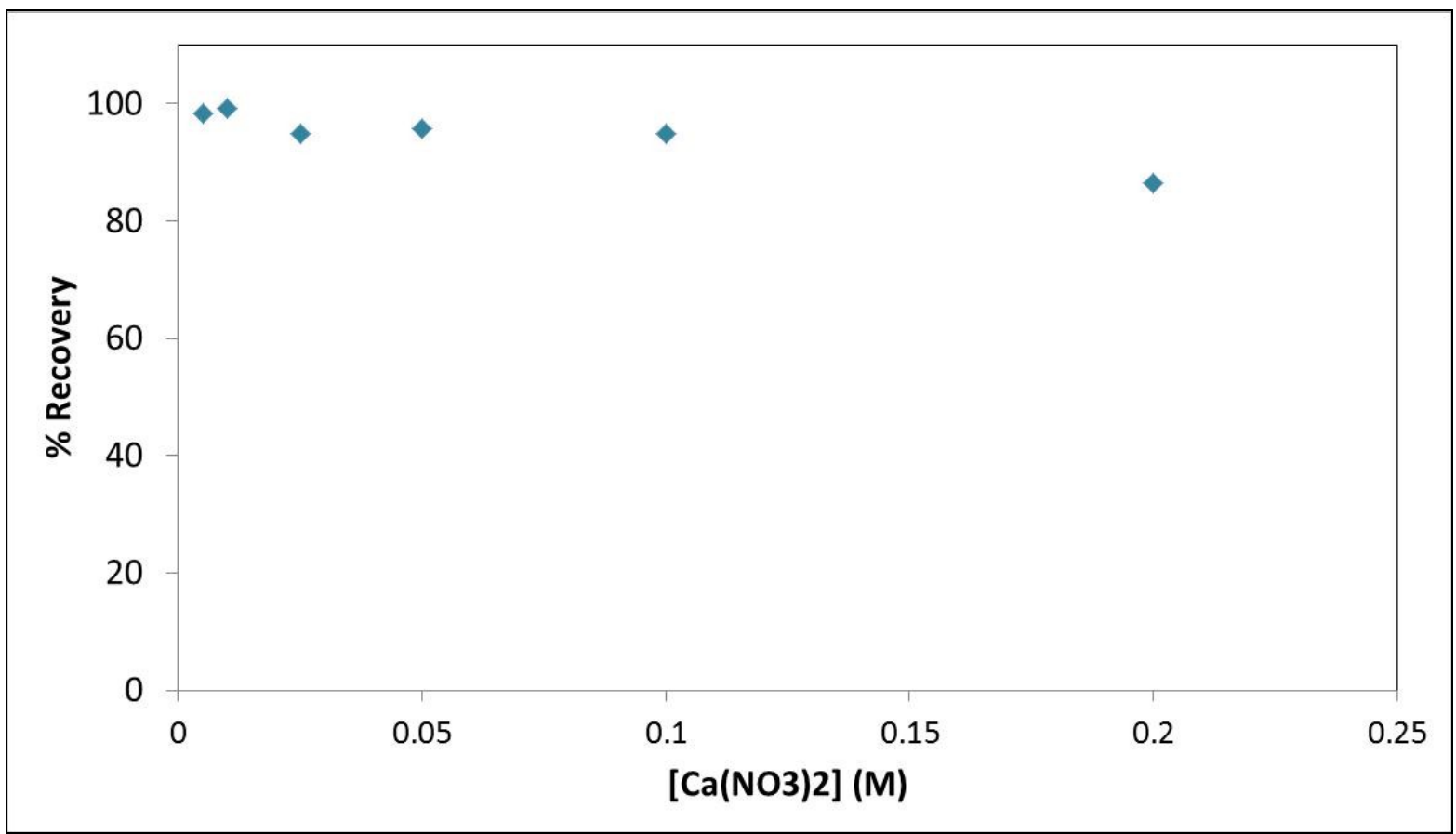

Figure 8: Effect of salt on extraction.

\subsection{Effect of centrifugation time}

Once a dispersion has formed within the sample, centrifugation is required for phase separation as it will take over 30 minutes to phase separation by gravity alone. It has been reported that centrifugation times of 5-20 minutes is sufficient in most cases [5]. Nine samples were prepared similarly and every three samples were centrifuged for a different duration. There was no difference in the \% recovery (Table 3) and a centrifugation time of 5 minutes was chosen for future experiments.

Table 3: Effect of centrifugation Time

\begin{tabular}{ll}
\hline Centrifugation time (minutes) & Mean \% Recovery $(\mathbf{n}=\mathbf{3})$ \\
\hline 5 mins & $72.1 \pm 2.6$ \\
10 mins & $71.0 \pm 2.7$ \\
15 mins & $71.7 \pm 0.6$ \\
\hline
\end{tabular}

\subsection{Effect of sonication time}


A variant of DLLME called ultrasonication assisted DLLME, has reported enhancements in recovery when sonication is also applied to a sample after dispersing the extractant. To test if sonication improves recovery, similar samples were prepared with the only difference being sonication time (Table 4).

Table 4: Effect of sonication time

\begin{tabular}{ll}
\hline Sonication time (minutes) & Mean \% Recovery $(\mathbf{n}=\mathbf{3})$ \\
\hline 0 mins & $69.8 \pm 5.8$ \\
5 mins & $70.9 \pm 3.4$ \\
10 mins & $74.3 \pm 1.8$ \\
15 mins & $74.3 \pm 3.8$ \\
\hline
\end{tabular}

It was not clear if sonication was required or not, so a two-sample t-test was used to determine if there's a difference between no sonication and 15 minutes of sonication. The null hypothesis is no difference. A p-value of $0.4126>0.05$, was obtained leading to the conclusion of no statistical difference at the $95 \%$ confidence interval.

\subsection{Choice of scintillation cocktail}

Several scintillation cocktails were evaluated for their counting efficiency and ability to hold the sample in a uniform phase. The organic phase has no problem mixing with any of the scintillation cocktails but the aqueous back extractant is usually of high acidity. If the concentration of the acid in the sample exceeds $3 \mathrm{~mL}$ of $3 \mathrm{M} \mathrm{HNO}_{3}$ or $3 \mathrm{M}$ $\mathrm{HCl}$, the scintillation cocktail becomes milky instead of translucent and emissions of photons from the fluor will drop because of self-absorption within the vial.

Replicate extractions from the same urine stock were performed using an extraction mix of $2 \mathrm{~mL}$ methanol, $100 \mathrm{uL} \mathrm{HDEHP}$, and $25 \mathrm{uL}$ of $\mathrm{C}_{2} \mathrm{Cl}_{4}$. After extraction, the sample was added to either HiSafe 3 or Ultima Gold AB for pooled urine \#1. 
These were run immediately on the LSC and also re-ran 48 hours later to test the stability of the sample in the LSC cocktail. Because samples can only be counted one at a time, over 24 hours may pass before samples at the end of the rack are counted. There was no difference in the number of counts for HiSafe or Ultima Gold AB in pooled urine \#1 over the course of 48 hours.

It was noticed when pooled urine \#2 in HiSafe 3 was measured after 20 hours, the activity in the organic phase was less than $50 \%$ of the spiked amount and the combined activity of the organic phase and supernatant did not add up to $100 \%$. It was suspected that either a long wait before counting or an incompatibility of the urine matrix with the scintillation cocktail caused a decrease in counting efficiency. To test this, replicate extractions on pooled urine \#2 were performed using either HiSafe 3, Ultima Gold AB, Hi Ionic Fluor or Optiphase Supermix as the scintillation cocktail (Fig.9). This was repeated one more time with the first counting interval at 12 hours and HiSafe 3 was very similar to Ultima Gold AB, but it followed the same trend of decreasing after 12 hours (Fig 10). Table 5 lists the components from each of the tested scintillators. It appears that an absence of a secondary surfactant is causing the plutonium and urine matrix to precipitate within vial. This will lead to less interaction with the fluor, and a lower signal. Hi-Ionic Fluor was chosen as the scintillator based on availability in the lab and also stability. 
Table 5: Components of liquid scintillation cocktails

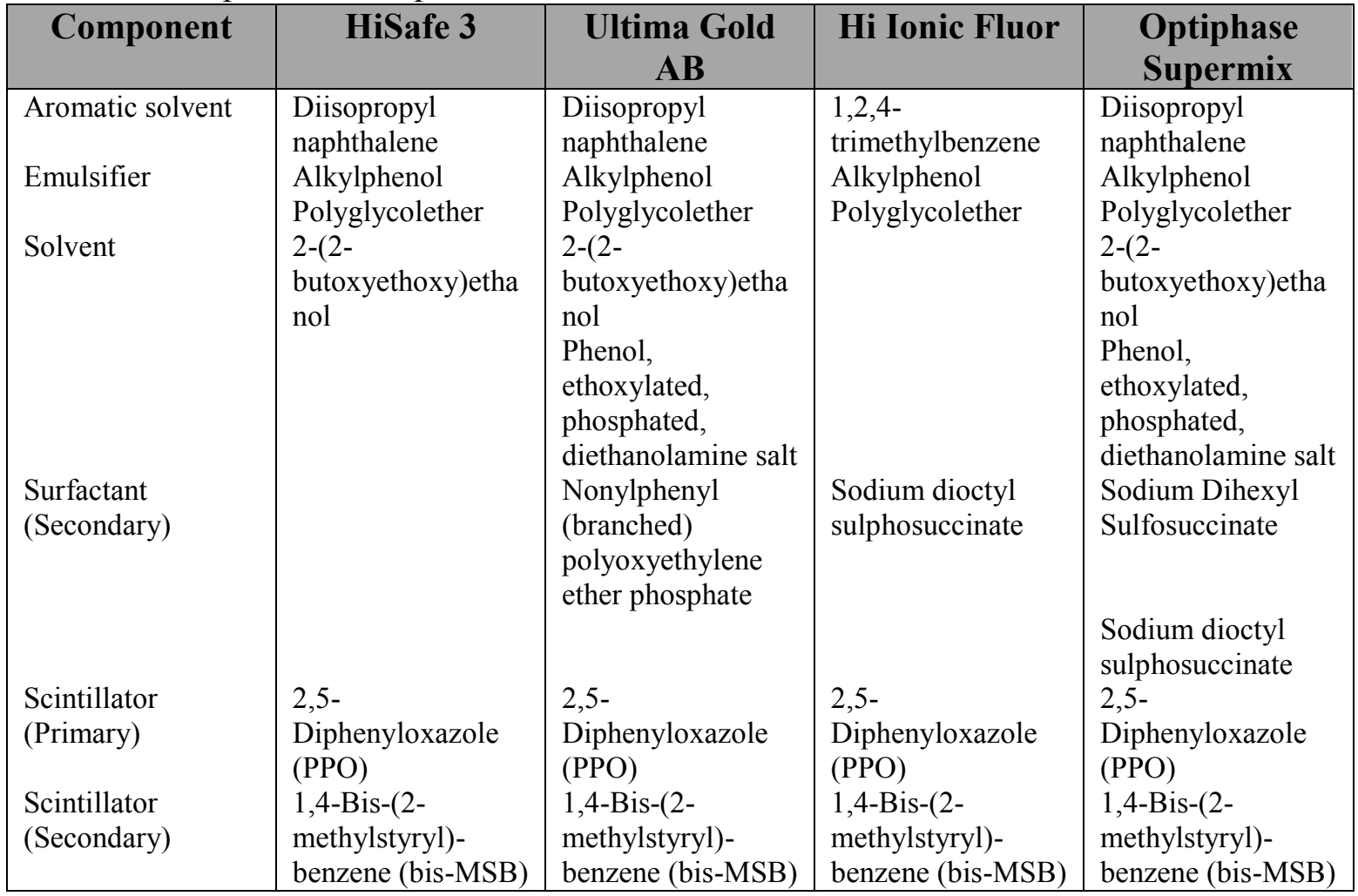

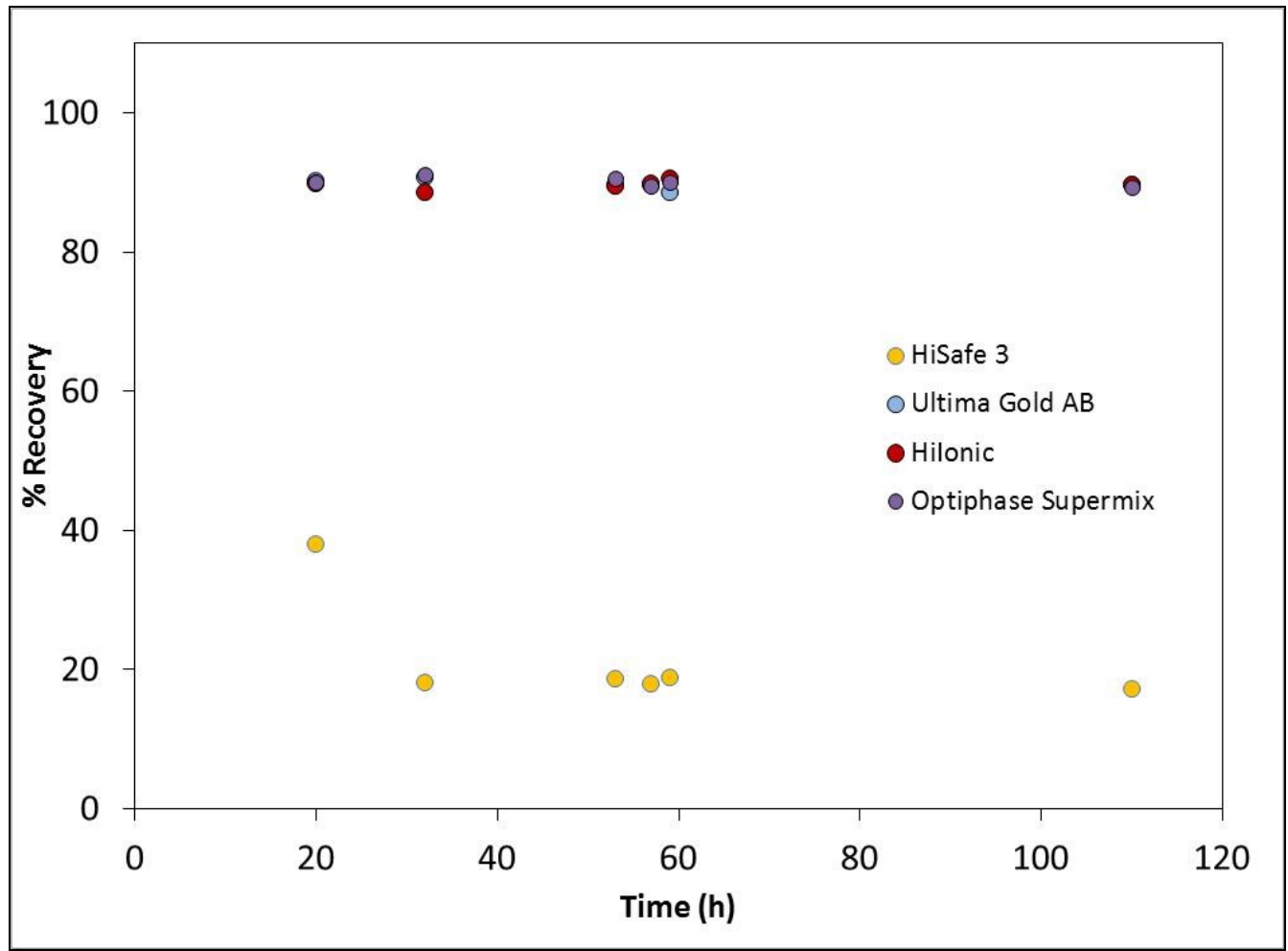

Figure 9: Effect of delayed counting in different LSC cocktails. 


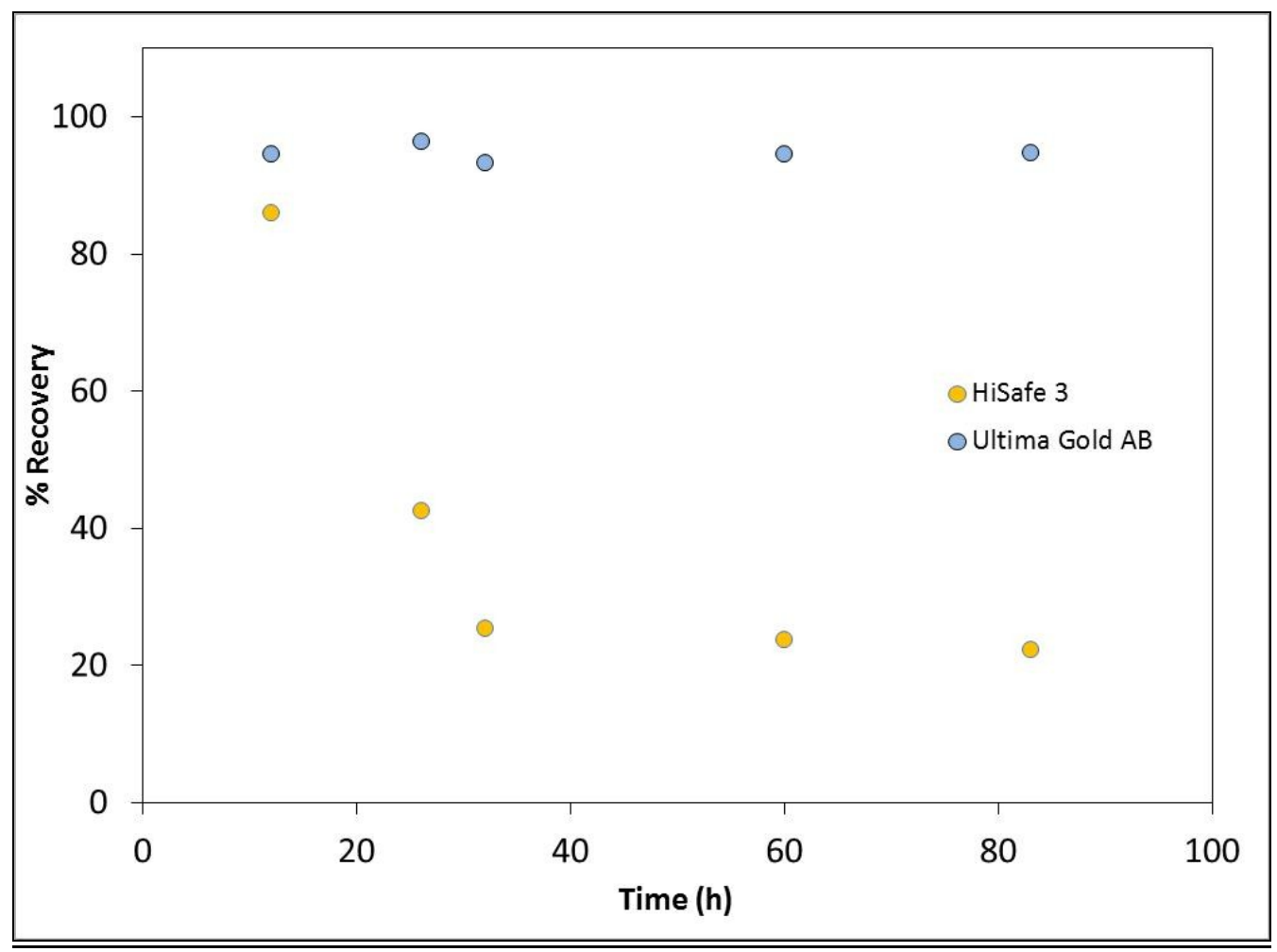

Figure 10: Effect of delayed counting in HiSafe 3 and Ultima Gold AB

\subsection{Effect of quenching agents}

Alpha emitters, due to their high energy, are less prone to quenching than beta emitters. However, it is still necessary to determine if the urine matrix will cause color quenching and if reagents in the extraction mix, such as $\mathrm{C}_{2} \mathrm{Cl}_{4}$, will cause chemical quenching. $\mathrm{C}_{2} \mathrm{Cl}_{4}$ quench was tested by adding $\sim 3 \mathrm{~Bq}$ of $\mathrm{Pu}-238$ into each of eight separate vials with varying amounts of $\mathrm{C}_{2} \mathrm{Cl}_{4}$ and measuring by $\mathrm{LSC}$ (Fig.11). $\mathrm{HNO}_{3}$ quench was done similarly by adding $\sim 3 \mathrm{~Bq}$ of $\mathrm{Pu}-238$ to each of twelve separate vials with varying amounts of concentrated $\mathrm{HNO}_{3}$ and measuring by LSC. Both $\mathrm{C}_{2} \mathrm{Cl}_{4}$ and $\mathrm{HNO}_{3}$ showed no chemical quenching on plutonium (Fig.12). For determination of color quenching, $2.5 \mathrm{~mL}$ of $\mathrm{C}_{2} \mathrm{Cl}_{4}$ was added to $100 \mathrm{~mL}$ of urine to concentrate the colored components of urine. From this concentrated matrix, varying amounts were added to an 
LSC vial containing $\sim 3 \mathrm{~Bq}$ of $\mathrm{Pu}-238$. The tSIE shows the presence of quench but there was no reduction in counts for the Pu-238 standard (Fig.13). Even though alpha counts did not drop, the quench parameter is useful in cases where Pu-241, a low energy beta emitter, needs to be measured.

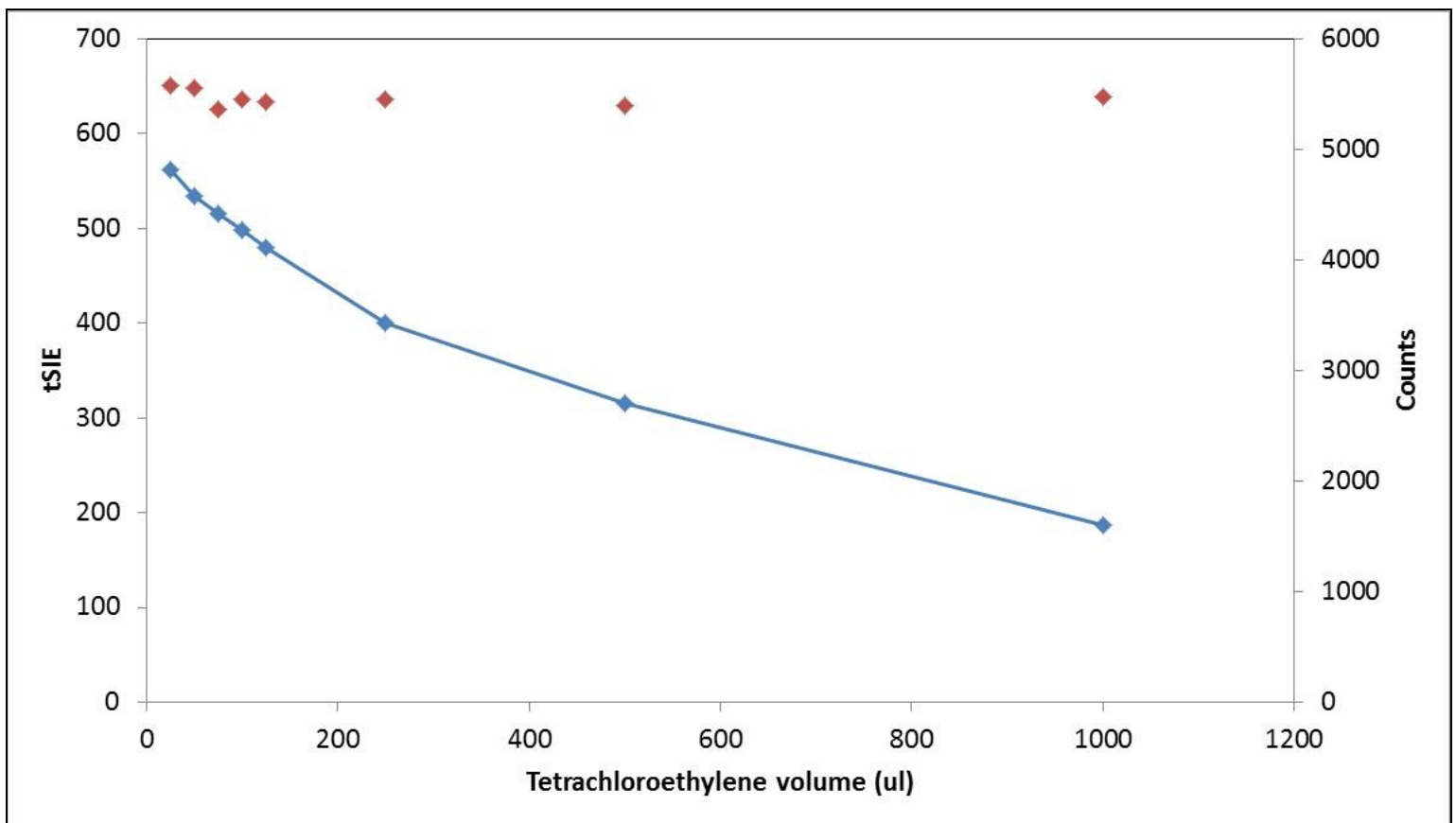

Figure 11 Effect of tetrachloroethylene on quenching parameter tSIE. 


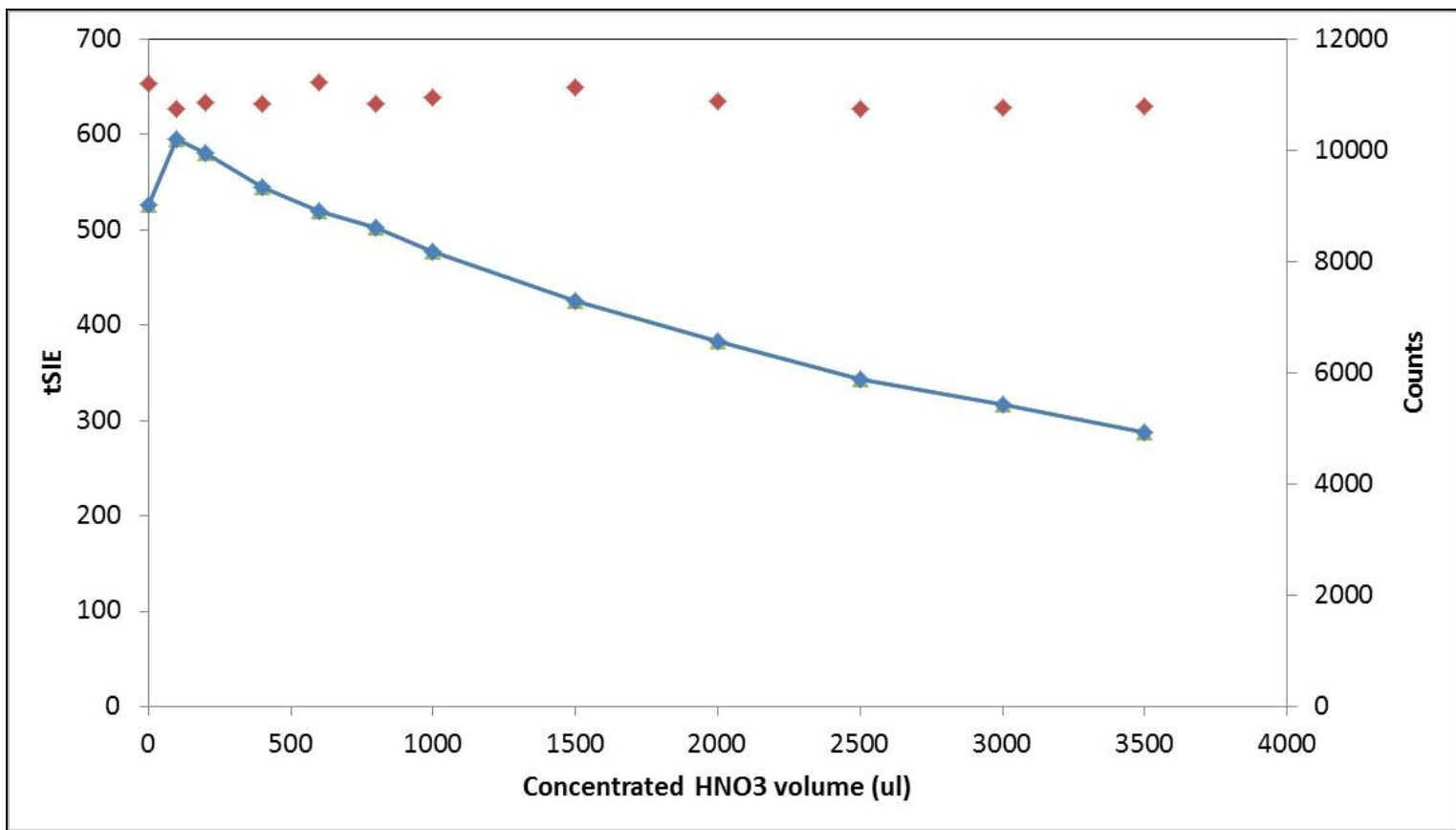

Figure 12: Effect of concentrated nitric acid on quenching parameter tSIE.

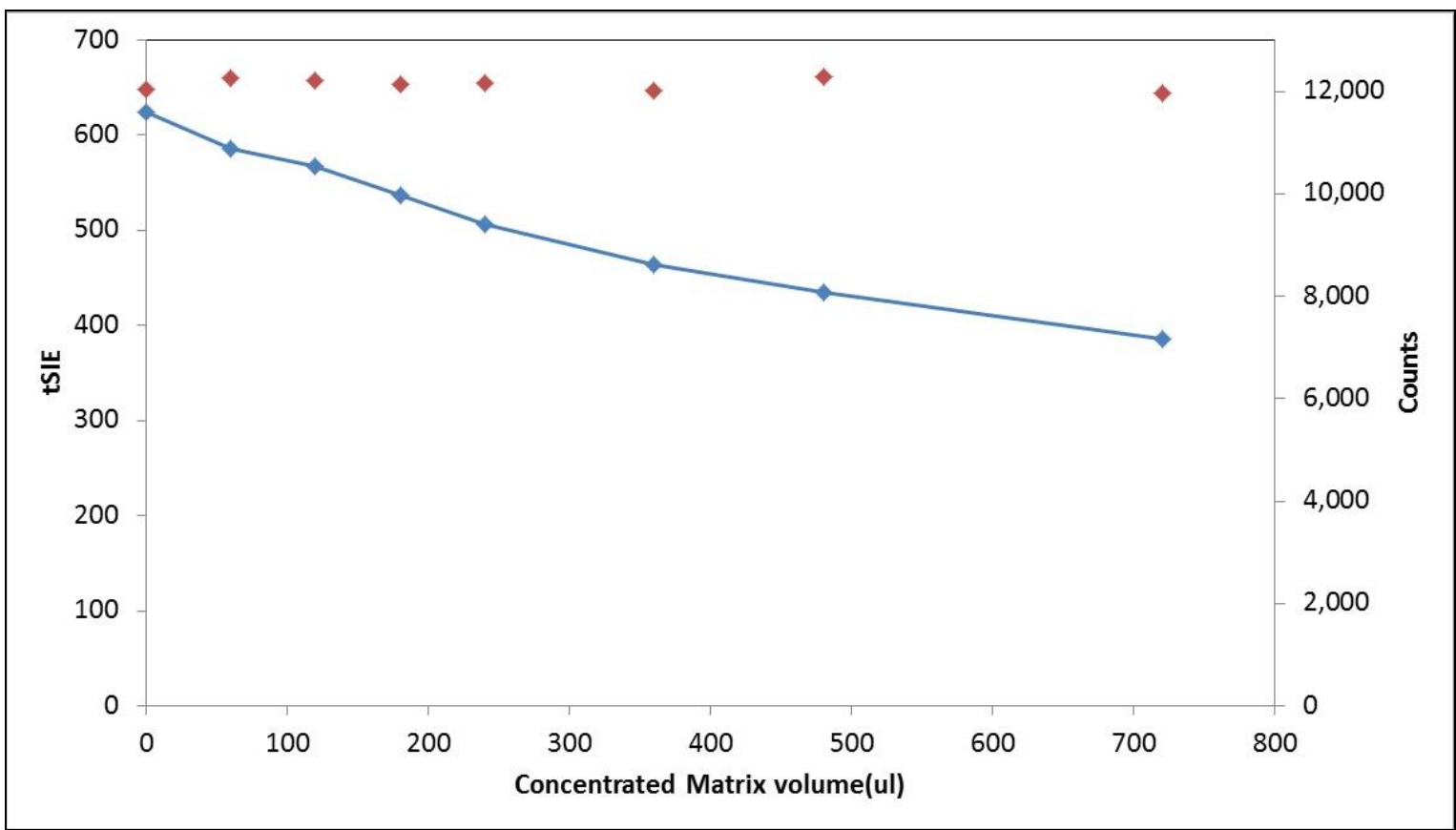

Figure 13: Effect of concentrated matrix on quenching parameter tSIE.

\subsection{Effect of valence adjustment}

HDEHP is known to have a higher affinity for actinides in the order of $+6>+4>$ +3 , as seen in the $\mathrm{pH}$ experiment. Varying amounts of sodium nitrite, an oxidizing agent, 
was added to the samples to achieve the concentrations shown in Figure 14. Nitrite concentration did not play a role in increasing the recovery; rather, the recovery appears to be lower than not adding any nitrite. This may be due to nitrite forming NO bubbles at high concentrations, and these bubbles may decrease the efficiency of dispersing the extraction mix.

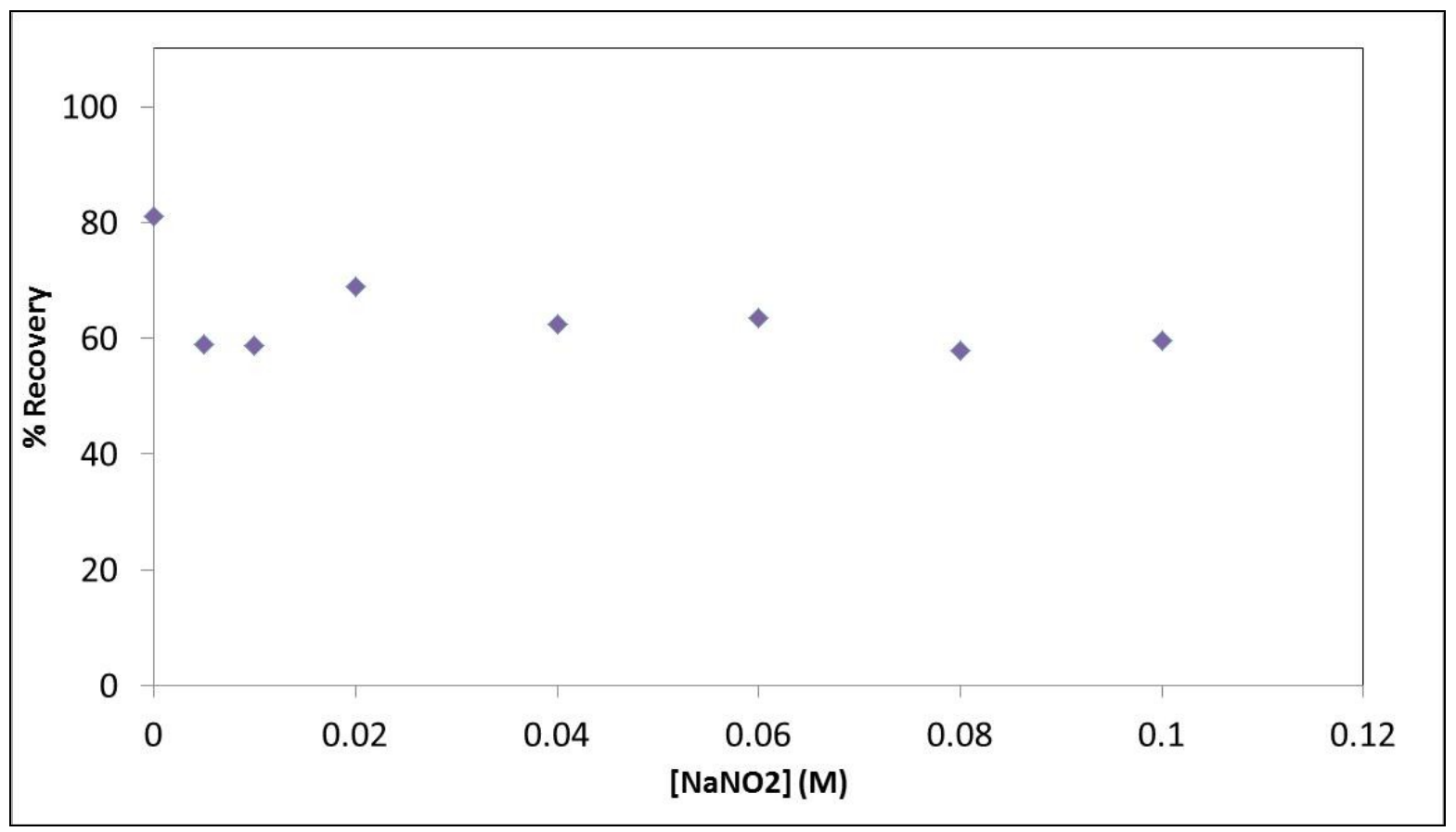

Figure 14: Effect of Valence Adjustment.

\section{$\underline{3.11 \text { Effect of sample volume }}$}

Spot samples of urine for emergency screening typically range from $10 \mathrm{~mL}$ to 100 $\mathrm{mL}$. To assess the efficacy of this DLLME for $40 \mathrm{~mL}$ urine samples, $8 \mathrm{~mL}$ of an extraction mix containing $400 \mathrm{uL} \mathrm{HDEHP}, 100 \mathrm{uL} \mathrm{C}_{2} \mathrm{Cl}_{4}$ in methanol was used. Initially, recoveries were less than $45 \%$ when using methanol to transfer the sedimented pellet to the LSC vial, within any further treatment. As seen in Section 3.8, low recoveries are suspect to be from the urine matrix precipitating out the $\mathrm{Pu}-\mathrm{HDEHP}$ from the scintillation cocktail. Because the volume of scintillator remains the same, whereas the urine matrix is 
now more concentrated, the amount of surfactant may not be enough to prevent precipitation.

Two commercial basic solubilizers, Soluene 350 and Solvable, typically used to dissolve soft tissue, blood, and urine samples prior to LSC measurement were tested for their ability to prevent the loss in signal with higher urine matrix loads. A set of extractions were performed on $40 \mathrm{~mL}$ samples with $3 \mathrm{~mL}$ of the solubilizers added after resuspension of the sedimented phase. Another set was performed with heating to see if this would have an effect. From the results, solvable can be used to digest extracts from $40 \mathrm{~mL}$ samples without heating.

Table 6: Effect of solubilizer post-extraction.

\begin{tabular}{lr}
\hline Solubilizer condition & $\begin{array}{r}\text { Mean \% Recovery } \\
(\mathbf{n}=\mathbf{3})\end{array}$ \\
\hline Methanol, No solubilizer & $40.0 \pm 2.0$ \\
Methanol, No solubilizer, Heating @ 90'C for 15 mins & $44.8 \pm 1.4$ \\
Methanol, Soluene 350 & $29.6 \pm 1.0$ \\
Methanol, Soluene 350, Heating @ 90'C for 15 mins & $31.6 \pm 2.8$ \\
Methanol, Solvable & $99.1 \pm 1.3$ \\
Methanol, Solvable, Heating @ 90'C for 15 mins & $97.5 \pm 0.9$
\end{tabular}

For spots samples of $100 \mathrm{ml}$ or more, it is possible to split them up into $40 \mathrm{ml}$ fractions, perform DLLME, and combine the sedimented phases. However, this can be time-consuming and will consume a high amount of reagents. A method employed for decades for the preconcentration of plutonium from bulk water or urine samples has been coprecipitation with an insoluble hydroxide, phosphate, or sulfate. Calcium phosphate is 
the most common precipitation agent but it was suspected that the high phosphate content can later inhibit extraction of plutonium.

Six calcium phosphate precipitations were done on different pooled urine samples and unpooled urine. Because the recovery is low (50\% on average) and variable, calcium phosphate precipitation coupled with the DLLME and LSC is not an option. Instead, alpha spectrometry may be used. A tracer can be used to track the loss of the analyte. (Table 7).

Table 7 Average Recovery of $\mathrm{Pu}$ activity from $\mathrm{Ca}_{3}\left(\mathrm{PO}_{4}\right)_{2}$

\begin{tabular}{ll}
\hline Urine Sample & \% Recovery \\
\hline U1 & 55.1 \\
U2 & 42.6 \\
U3 & 52.3 \\
U4 & 38.0 \\
U5 & 62.5 \\
U6 & 71.4 \\
\hline Average & $53.6 \pm 12.4$ \\
\hline
\end{tabular}

\section{$\underline{3.12}$ Back extraction}

There are three main methods to back extract a metal from an acidic extractant: 1) vary the acid and/or acidity of the aqueous solution in contact with the extractant; 2) add an aqueous complexing agent with higher affinity for plutonium than HDEHP; and 3) use valence adjustment to reduce plutonium to +3 , which should have lower affinity for HDEHP.

Phosphoric acid was tested as a back extractant from the knowledge that plutonium is very poorly extracted from high concentration of phosphoric acid (Fig.15). 


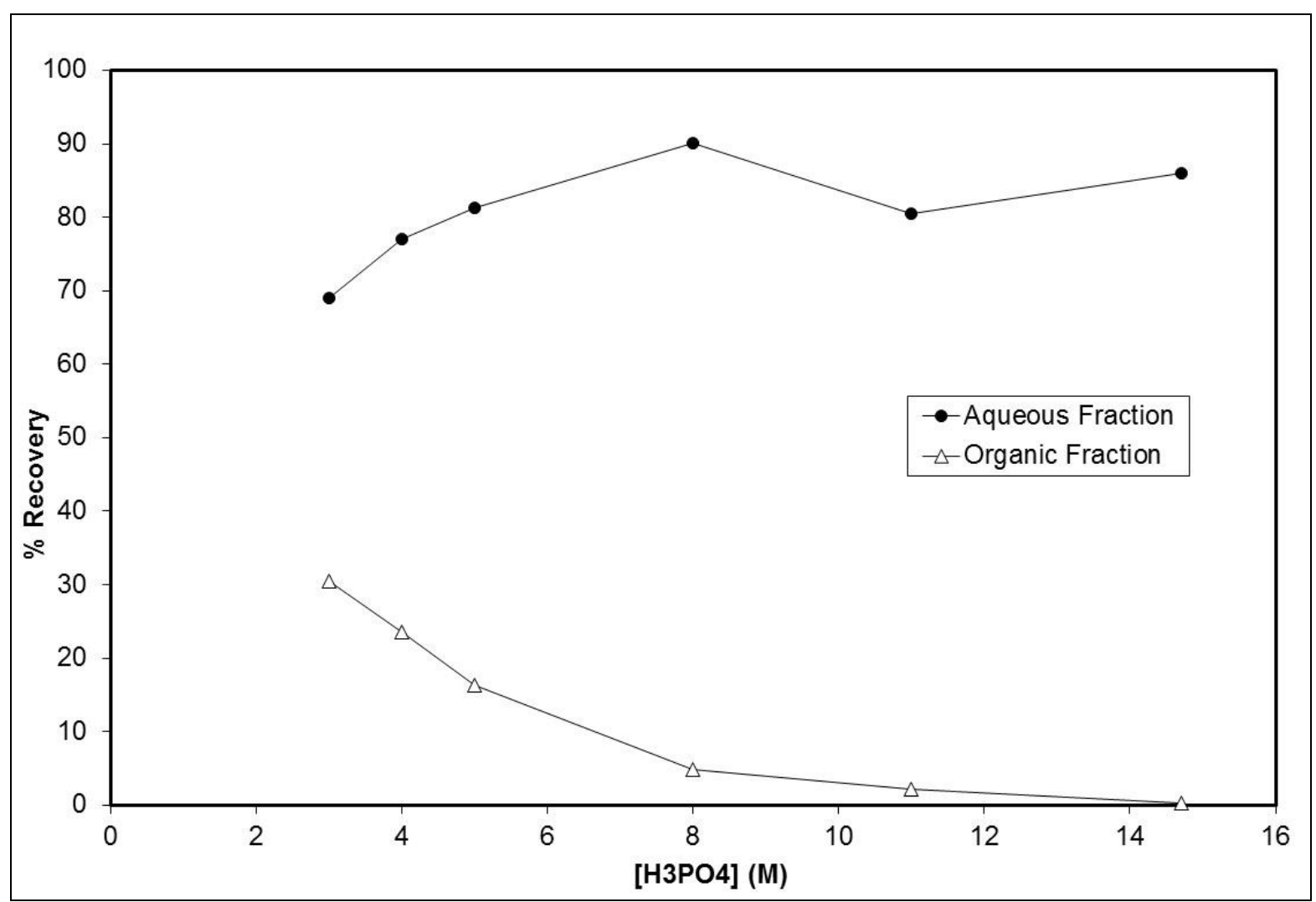

Figure 15. Back extraction of Pu-HDEHP from phosphoric acid media.

Sulfuric acid gave less than $60 \%$ back extraction efficiency and the sample had to be split into multiple LSC vials because sulfuric acid reacts with the scintillation cocktail to form a milky suspension that is no longer measurable by LSC.

For the back extraction of $\mathrm{Pu}-239$ along with a potential tracer, Pu-242, using a complexing agent, $0.2 \mathrm{M}$ sodium oxalate, triplicate samples was performed and measurement was done by both LSC and alpha spectrometry. For comparison, another back extraction was done using $2 \mathrm{M} \mathrm{HCl}$, containing a $0.2 \mathrm{M}$ ascorbic acid, which is known to be compatible with LSC [32].

Initially, it appears that oxalate is better than $2 \mathrm{M} \mathrm{HCl}+0.2 \mathrm{M}$ ascorbic when measured by LSC (Fig.16). However, on the alpha spectrum, the oxalate sample had much poorer intensity and resolution. This can be due to an incompatibility of the oxalate 
with the microprecipitation mixture causing insoluble oxalates to form, and increasing the self-absorption within the sample (Fig. 17, 18).

Further back extraction studies focused on finding the optimal back extraction conditions for $\mathrm{Pu}$ by using a range of $\mathrm{HCl}$ concentration with ascorbic acid, and also one without. Based on (Fig. 19), a back extraction condition of $2 \mathrm{M}$ to $3 \mathrm{M} \mathrm{HCl}+0.2 \mathrm{M}$ ascorbic was chosen.

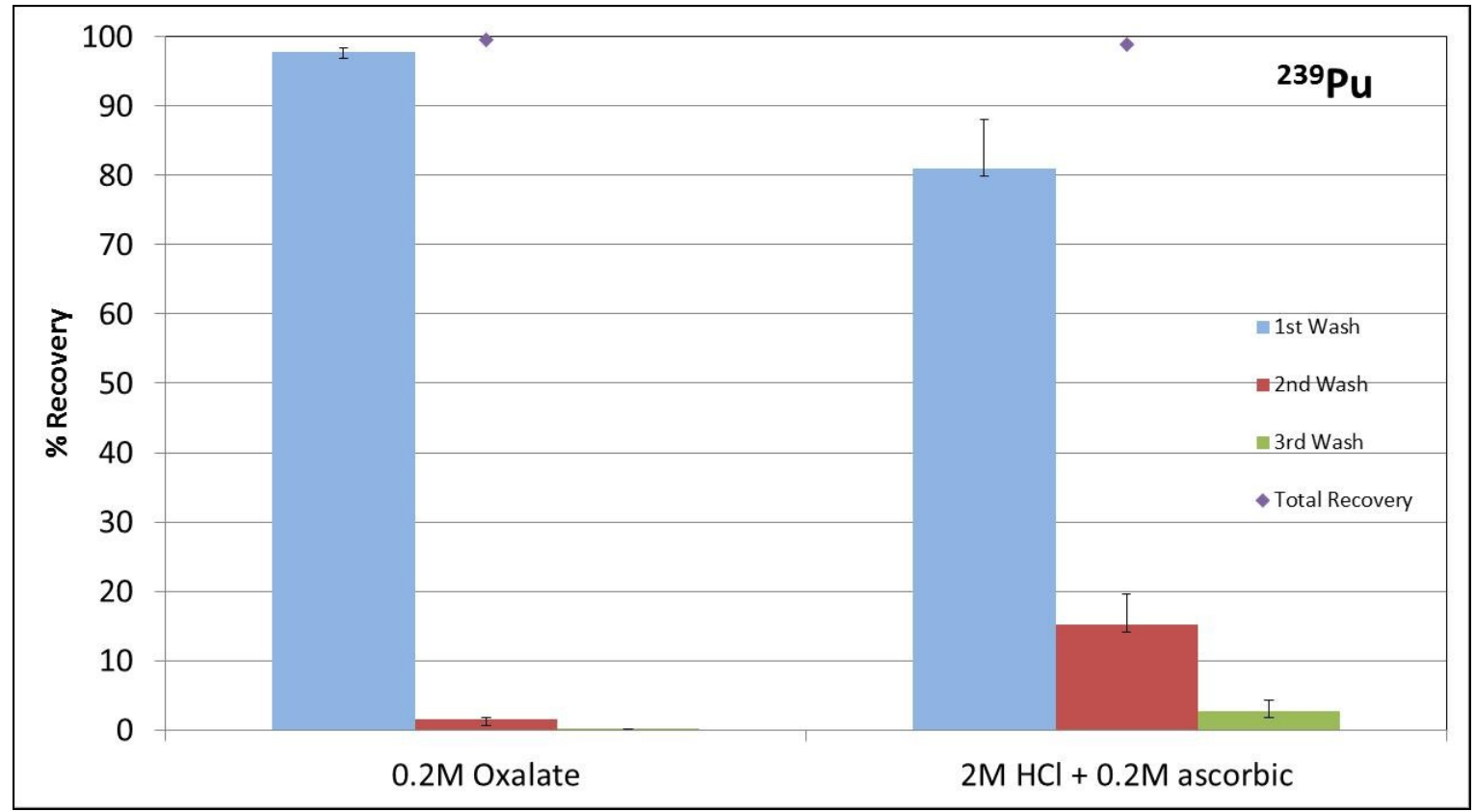

Figure 16: Back extraction of plutonium from HDEHP 


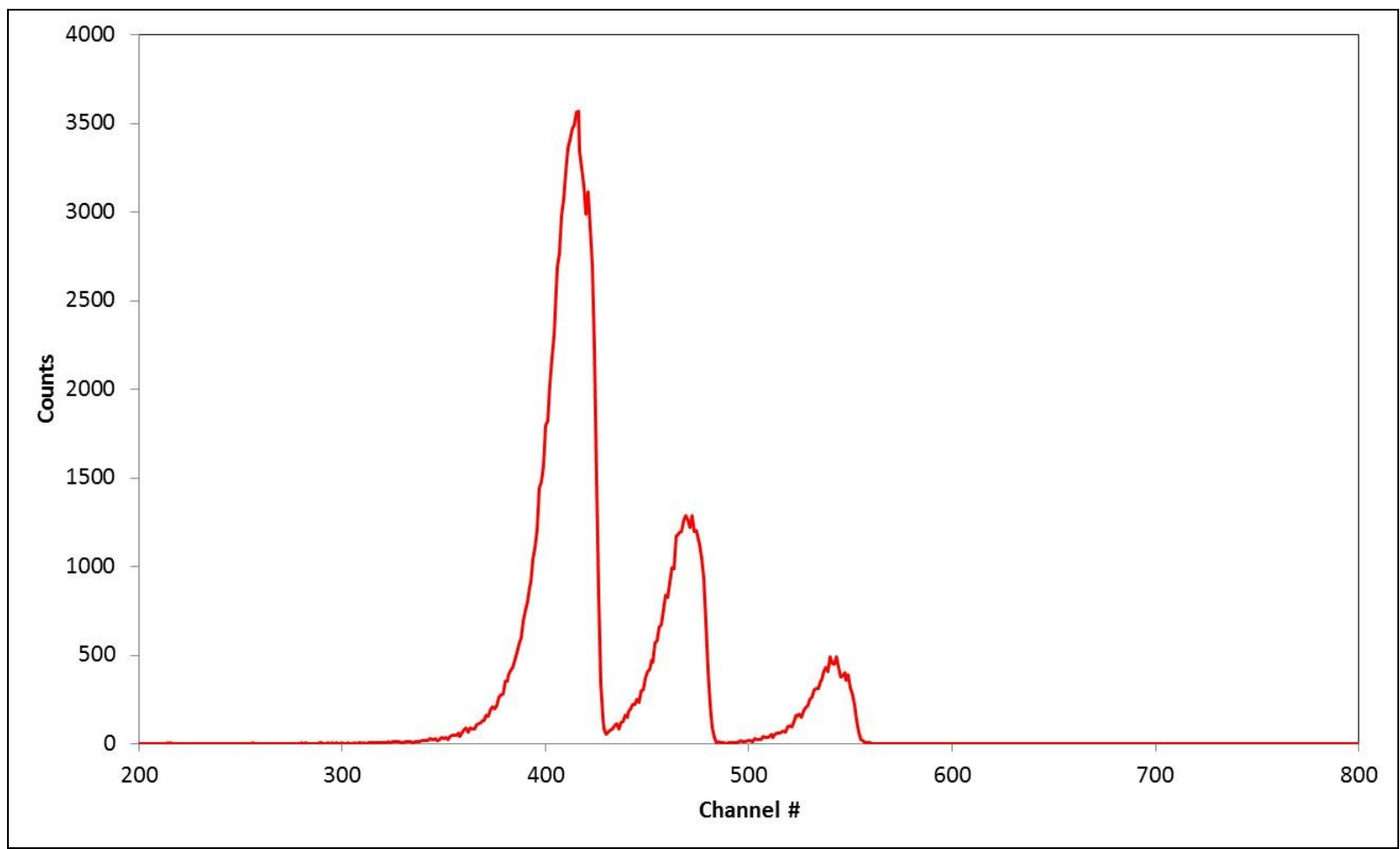

Figure 17: Alpha spectrum for back extraction using $2 \mathrm{M} \mathrm{HCl}$ and $0.2 \mathrm{M}$ ascorbic.

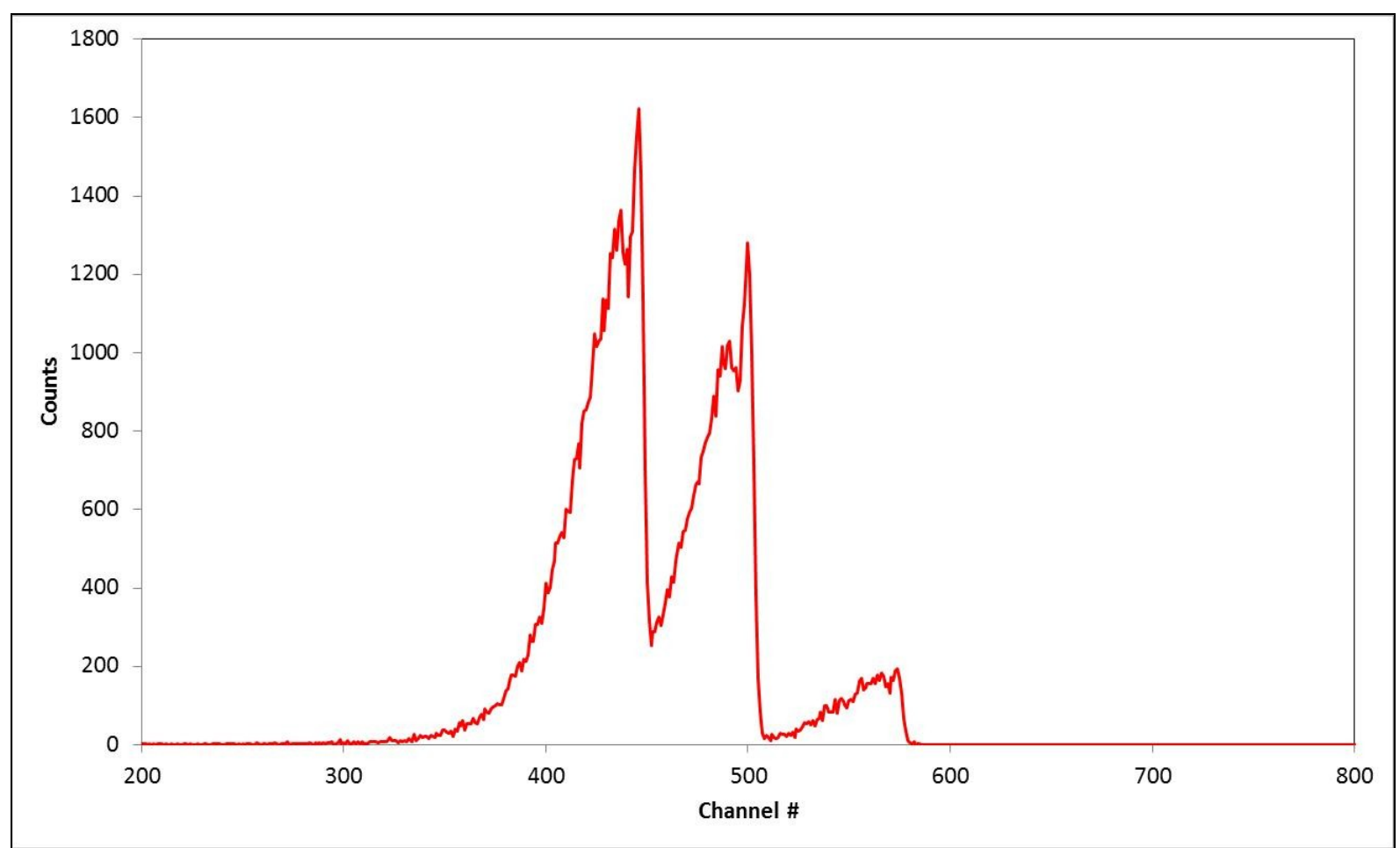

Figure 18: Alpha Spectrum for back extraction with 0.2M Oxalate 


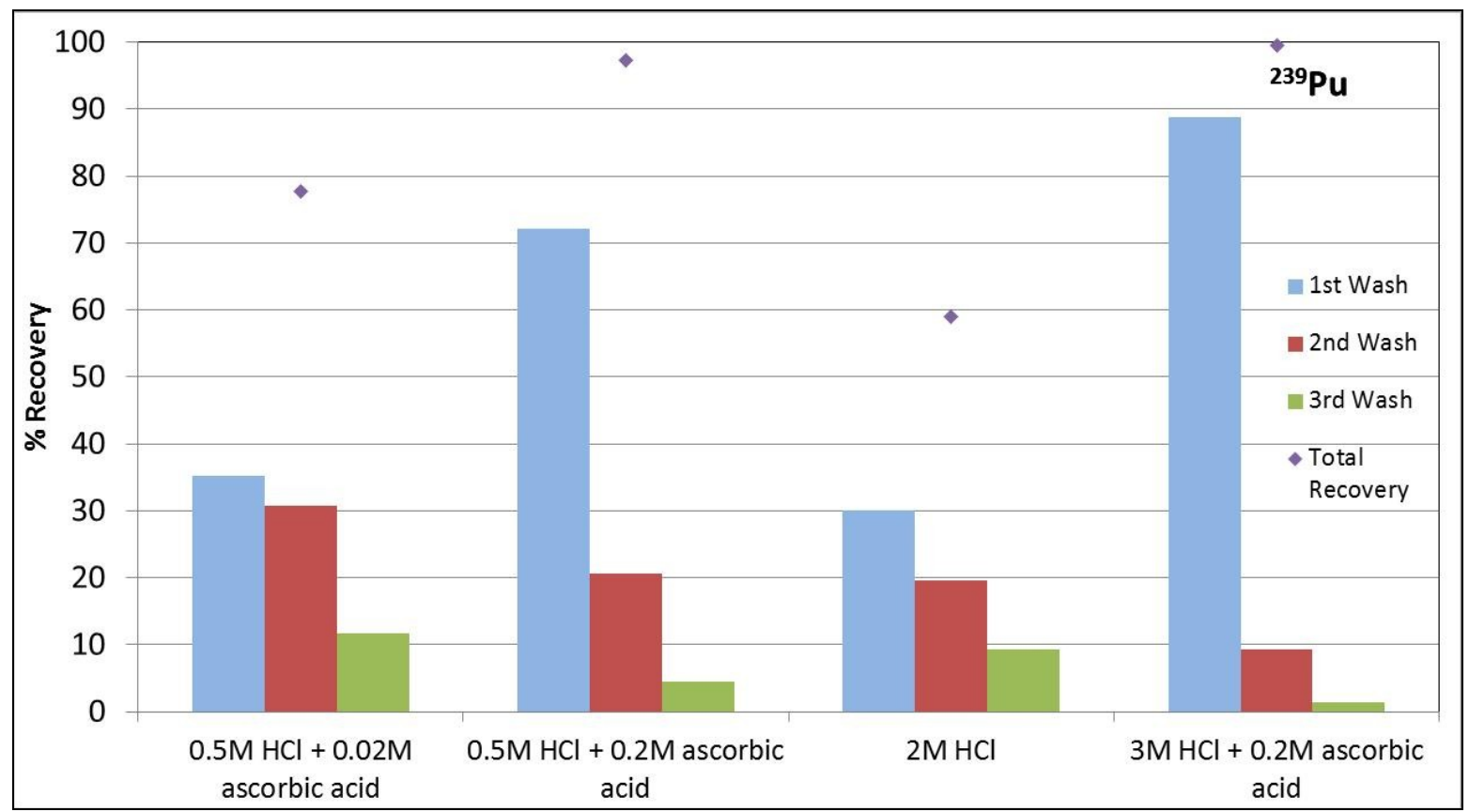

Figure 19: Back Extractants For Pu

\section{$\underline{\text { 3.13 Back extraction of Am from Pu }}$}

The separation of Am from Pu is important because the alpha energy of Am-241 is very close to that of Pu-238 and the alpha energy of tracer Am-243 is very close to that of Pu-239. Different concentration of nitric acid was used to elute Am and $\mathrm{Pu}$ in separate experiments. It was determined that Am elutes in $2 \mathrm{M}$ HNO3 and sodium nitrite appears to be beneficial in retaining Pu with $\operatorname{HDEHP}$ (Fig. 20, 21). Using alpha spectroscopy, a $1 \mathrm{M} \mathrm{HNO}_{3}+0.02 \mathrm{M} \mathrm{NaNO}_{2}$ wash was tried on sedimented pellet containing both Am-241 and $\mathrm{Pu}-239$. Doing 3 washes with $2 \mathrm{~mL}$ each was able to remove $71 \pm 17 \%$ of $\mathrm{Am}-241$ and $3.7 \pm 4.8 \%$ of $\mathrm{Pu}-239$. When the back extractant acid was switched from $1 \mathrm{M} \mathrm{HNO}_{3}$ to $0.5 \mathrm{M} \mathrm{HCl}$, the recovery of $\mathrm{Pu}-239$ in the americium fraction was decreased to near zero in the presence of $0.01 \mathrm{M} \mathrm{NaNO}_{2}$ (Fig. 22), while $93 \%$ of the americium was back extracted in $3 \times 2 \mathrm{ml}$ washes. The plutonium back extraction condition of $2 \mathrm{M} \mathrm{HCl}+0.2 \mathrm{M}$ ascorbic acid mentioned in 3.12 gave much lower recovery if it is preceded with the 
americium back extraction condition because much of the plutonium has been oxidized by $\mathrm{NaNO}_{2}$. To effectively back extract Pu after Am, a back extraction condition of $0.5 \mathrm{M}$ $\mathrm{NaHCO}_{3}$ and $1 \mathrm{M}$ hydrazine was chosen. At near neutral $\mathrm{pH}, \mathrm{Pu}$ can form carbonate complexes, and also have low affinity for HDEHP. The hydrazine serves as the reducing agent which can exist in higher concentrations in aqueous solutions compared to ascorbic acid. This back extraction condition was able to extract $91 \%$ of Pu after the Am back extraction (Table 8).

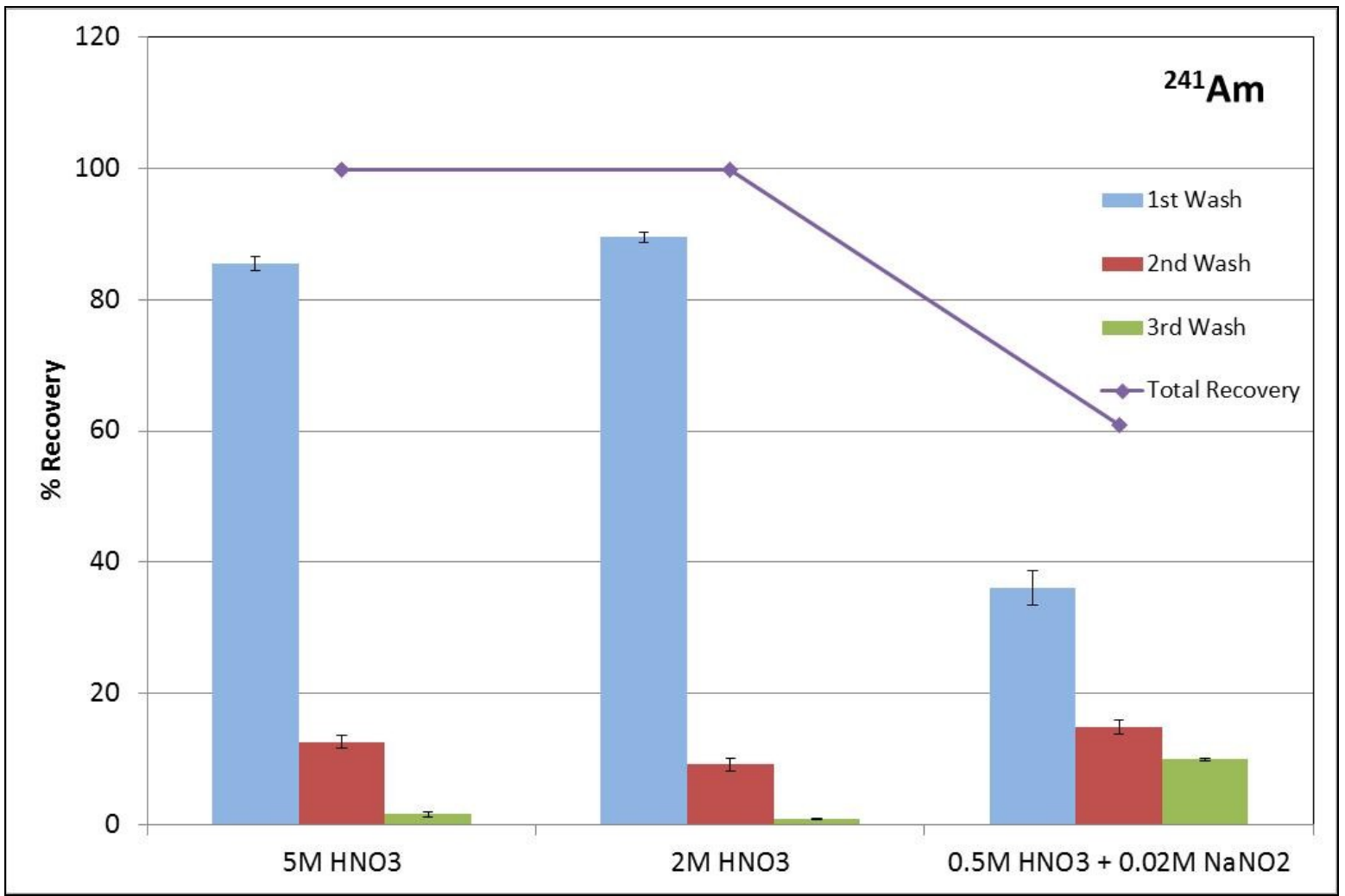

Figure 20: Back extraction of Am from nitric acid media. 


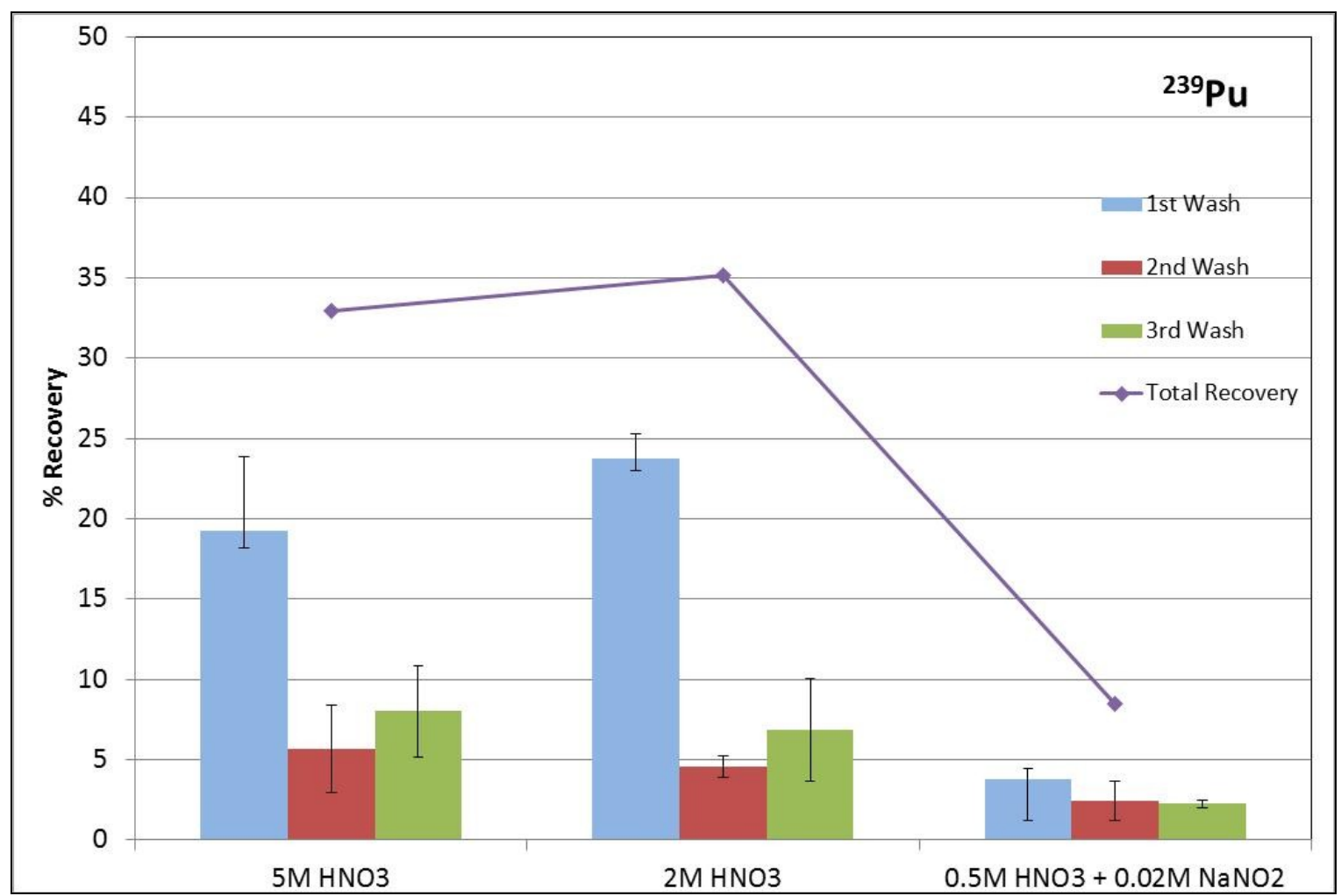

Figure 21: Back extraction of $\mathrm{Pu}$ from nitric acid media.

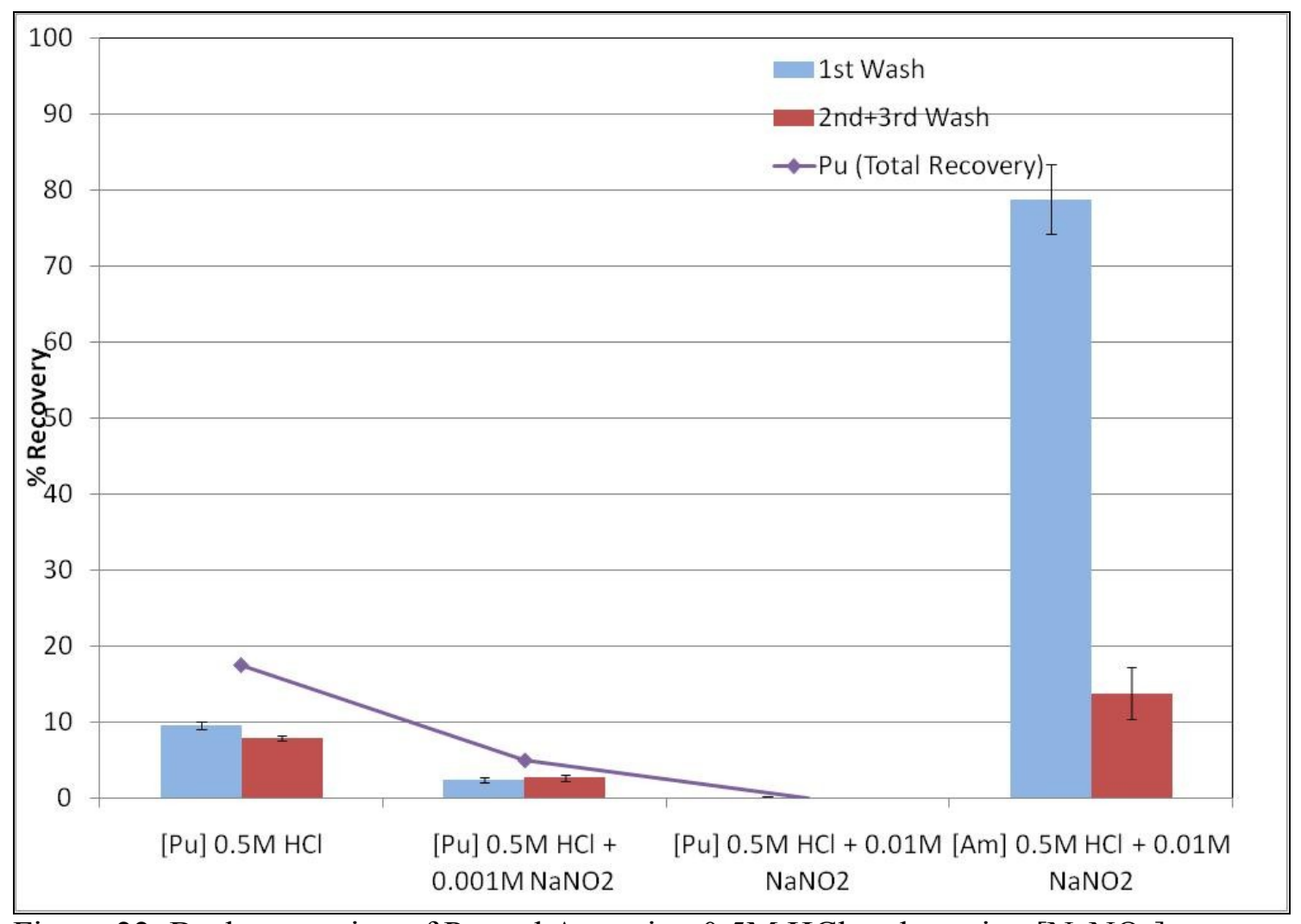

Figure 22. Back extraction of $\mathrm{Pu}$ and $\mathrm{Am}$ using $0.5 \mathrm{M} \mathrm{HCl}$ and varying $\left[\mathrm{NaNO}_{2}\right]$. 
Table 8 Sequential back extraction recoveries for urine containing both $\mathrm{Pu}$ and Am

\begin{tabular}{|c|c|c|}
\hline Back Extactant & $\%$ Recovery Pu $(n=3)$ & $\%$ Recovery Am $(n=3)$ \\
\hline $0.5 \mathrm{M} \mathrm{HCl}+0.01 \mathrm{M} \mathrm{NaNO}_{2}$ & $3.4 \pm 1.1$ & $87.9 \pm 10.8$ \\
\hline $0.5 \mathrm{M} \mathrm{NaHCO} \mathrm{N}_{3}+1 \mathrm{M}$ hydrazine & $91.1 \pm 2.6$ & $1.3 \pm 0.3$ \\
\hline Total Recovery & $94.4 \pm 2.1$ & $89.3 \pm 10.6$ \\
\hline
\end{tabular}

\section{$\underline{\text { 3.14 DLLME on Pu-DTPA }}$}

Fenton's reagent has been used in previous studies to oxidize EDTA to $60-75 \%$ completion [24]. Samples containing Pu-DTPA were first adjusted to $\mathrm{pH} 7-11$, where report to be the optimal range for the reaction with maximum yield around $\mathrm{pH} 7-8$. Then $\mathrm{FeCl}_{3}$ was added to achieve a final concentration of $0.025 \mathrm{M}$ and $30 \% \mathrm{H}_{2} \mathrm{O}_{2}$ was added to achieve a final concentration of $3 \% \mathrm{v} / \mathrm{v}$. The mixture was sonicated for 30 minutes before the normal extraction was performed. Under all $\mathrm{pH}$ conditions, the \% recovery of plutonium did not exceed 30\% (Table 9).

Table 9: Effect of Fenton's reagent on recovery of Pu-DTPA

\begin{tabular}{ll}
\hline Fenton's reaction $\mathbf{p H}$ & \% recovery \\
\hline 7 & 27.6 \\
8 & 24.1 \\
9 & 21.0 \\
10 & 24.1 \\
11 & 16.5 \\
\hline
\end{tabular}

It was suspected that either the reaction is unsuccessful in urine because there are many oxidizable compounds in urine or the quantity of reagents may not be enough. Furthermore, it is possible that the $\mathrm{Fe}^{3+}$ is also captured by HDEHP during the later stages of the extraction and since it is colored, it can reduce the LSC signal. It was shown in section 3.9, that slight coloration of the LSC color did not decrease the plutonium 
signal. There is a threshold where color compounds block out the light entirely. For instance, the other reported oxidation agent, $\mathrm{KMnO}_{4}$, is dark purple, and forms very dark mixtures with the scintillation cocktail, and as a result, its counts on LSC are close to background.

An alternate oxidizing agent, potassium persulfate was investigated because it had key properties such as a high redox potential, compatibility with the LSC cocktail, and non-reactivity with the reagent mixture. Three sets of samples containing Pu-DTPA were created: one without heating and $\mathrm{S}_{2} \mathrm{O}_{8}{ }^{2-}$, one with 4 hour heating and no $\mathrm{S}_{2} \mathrm{O}_{8}{ }^{2-}$, and one with 2 hour heating and $\mathrm{S}_{2} \mathrm{O}_{8}{ }^{2-}$. Heating was used because of greater oxidation of the related compound, EDTA, was shown at higher temperatures [33]. However, heating did not show an increase in recovery whereas persulfate increased the recovery to $63 \%$. Because the recovery is relatively low with a high standard deviation, a Pu-DTPA tracer (either 242 or 236), should be added before the oxidation process, to track the recovery and analysis should be done on alpha spectroscopy only.

Table 10: Effect of persulfate on DTPA oxidation

\begin{tabular}{ll}
\hline Oxidization Condition & Mean \% recovery $(\mathbf{n}=\mathbf{5})$ \\
\hline None & $17.1 \pm 3.3$ \\
Heat @ 90'C for 4 hours & $18.7 \pm 4.1$ \\
Heat @ 90'C for 4 hours, $0.075 \mathrm{M} \mathrm{K}_{2} \mathrm{~S}_{2} \mathrm{O}_{8}$ & $62.7 \pm 14.4$ \\
\hline
\end{tabular}

\section{$\underline{3.15 \text { Evaluation of replicate urine samples }}$}

Samples for method development have used pooled urine, but it is also necessary to test the variation of multiple donors to assess the effect of matrix on extraction. $40 \mathrm{~mL}$ of spot urine was collected from each of eight individuals and was acidified with $1 \% \mathrm{HCl}$ on the day of collection, spiked with Pu-238. DLLME and LSC counting were performed 
within 48 hours of collection. Results ranged from $72-90 \%$ recovery. It is hypothesized that the lower recoveries from Donor 1, 3, and 4 can be a result of increased dissolved solids in the matrix prevented uniform dispersion, or as a result of other competing complexants in the urine such as oxalic or citric acids. The average recovery from all 8 donors is $84.4 \pm 2.3 \%$. Tap water was also used to see if the optimized method was applicable to water samples. Recoveries of $95.4 \pm 0.7 \%$ were achieved for tap water (Fig. 23)

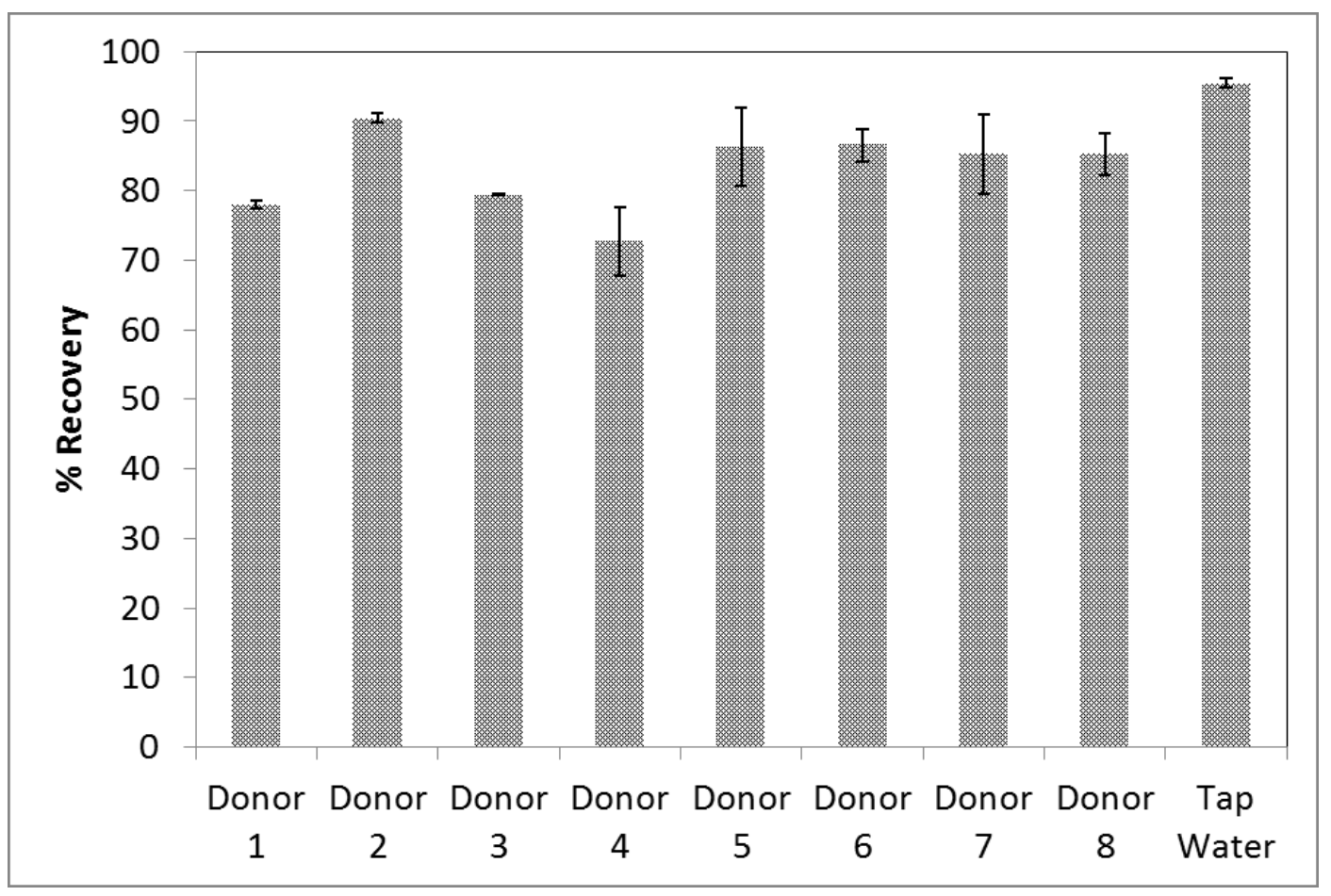

Figure 23. Replicate samples from eight urine types.

\subsection{Optimized flow procedures}

Three optimized flow procedures were produced depending on scenarios. When DLLME is used for screening a single isotope of plutonium in a small volume $(<40 \mathrm{ml})$ sample, then the procedure in Figure 27 can be used. In cases where there is a need to analyze a large volume of urine or isotopic plutonium, then the procedure in Figure 28 
can be used. Finally, the protocol in Figure 29 can be used when both americium and plutonium is present in the sample.

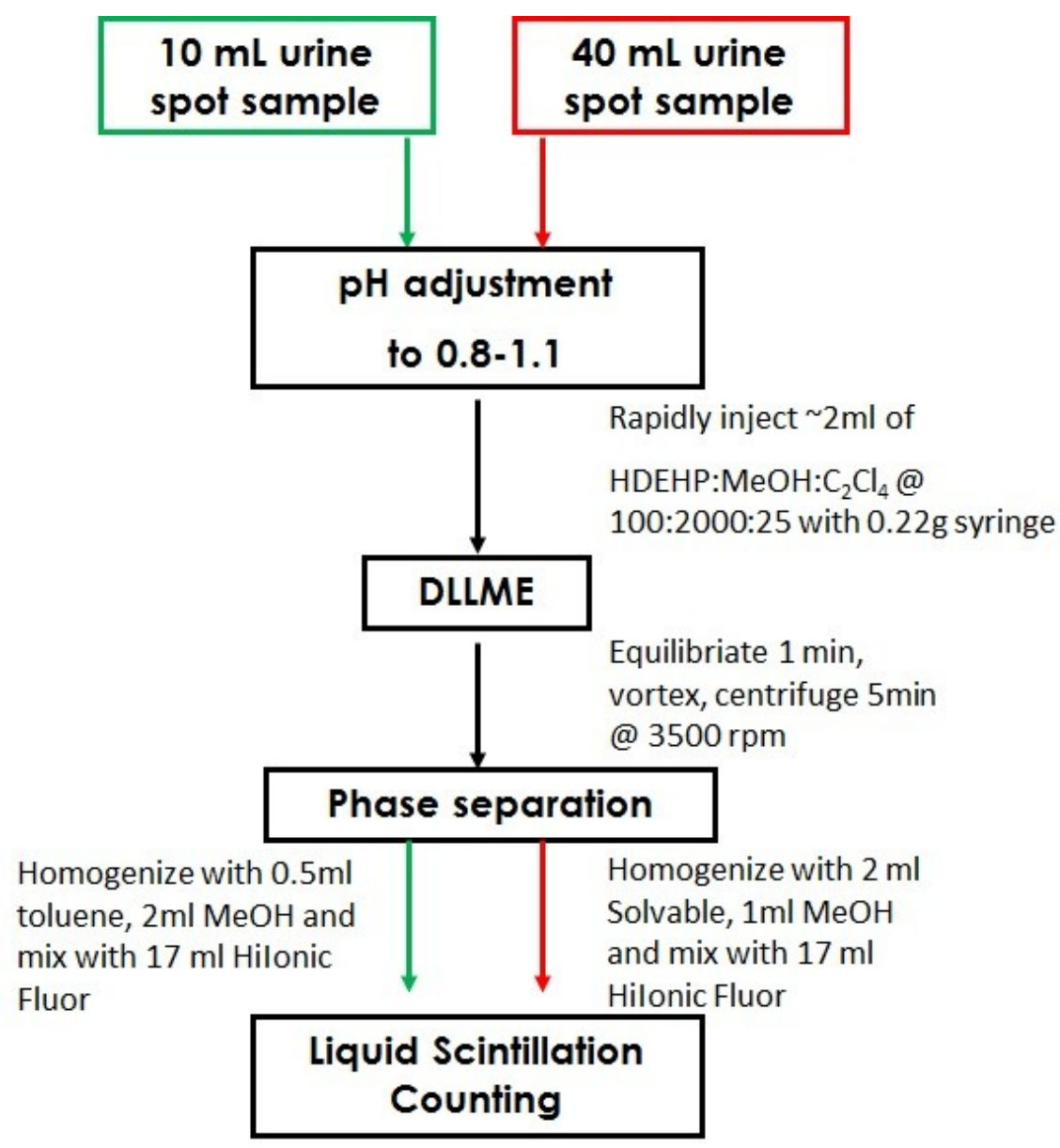

Figure 24. Flowchart for sample containing only one single isotope. Turnaround time = 30 minutes for preparation +30 minutes for counting $=1$ hour. 


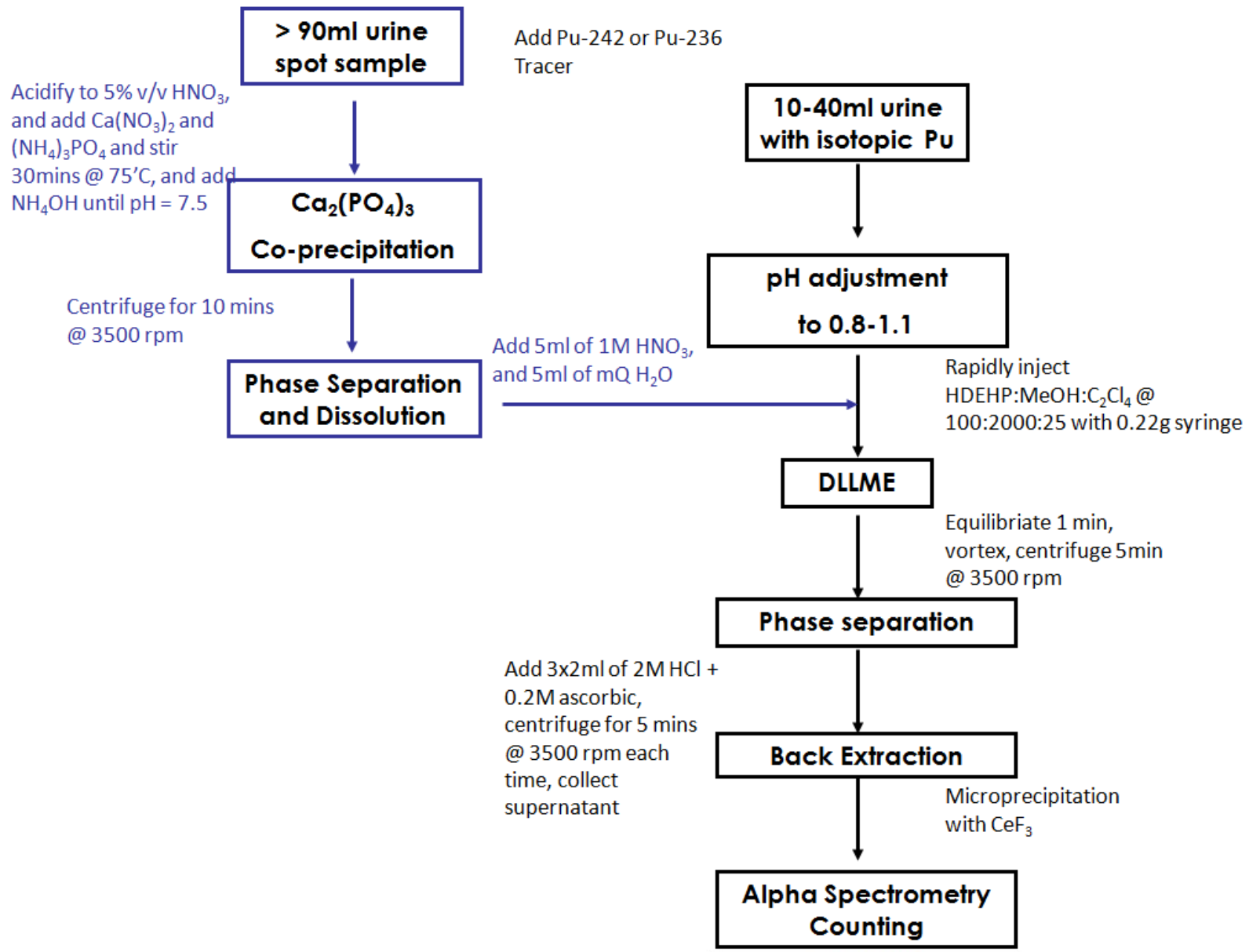

Figure 25: Flow chart for large samples and isotopic plutonium and low levels of plutonium. Turnaround time $=3.5$ hours for preparation + scenario-based counting time 


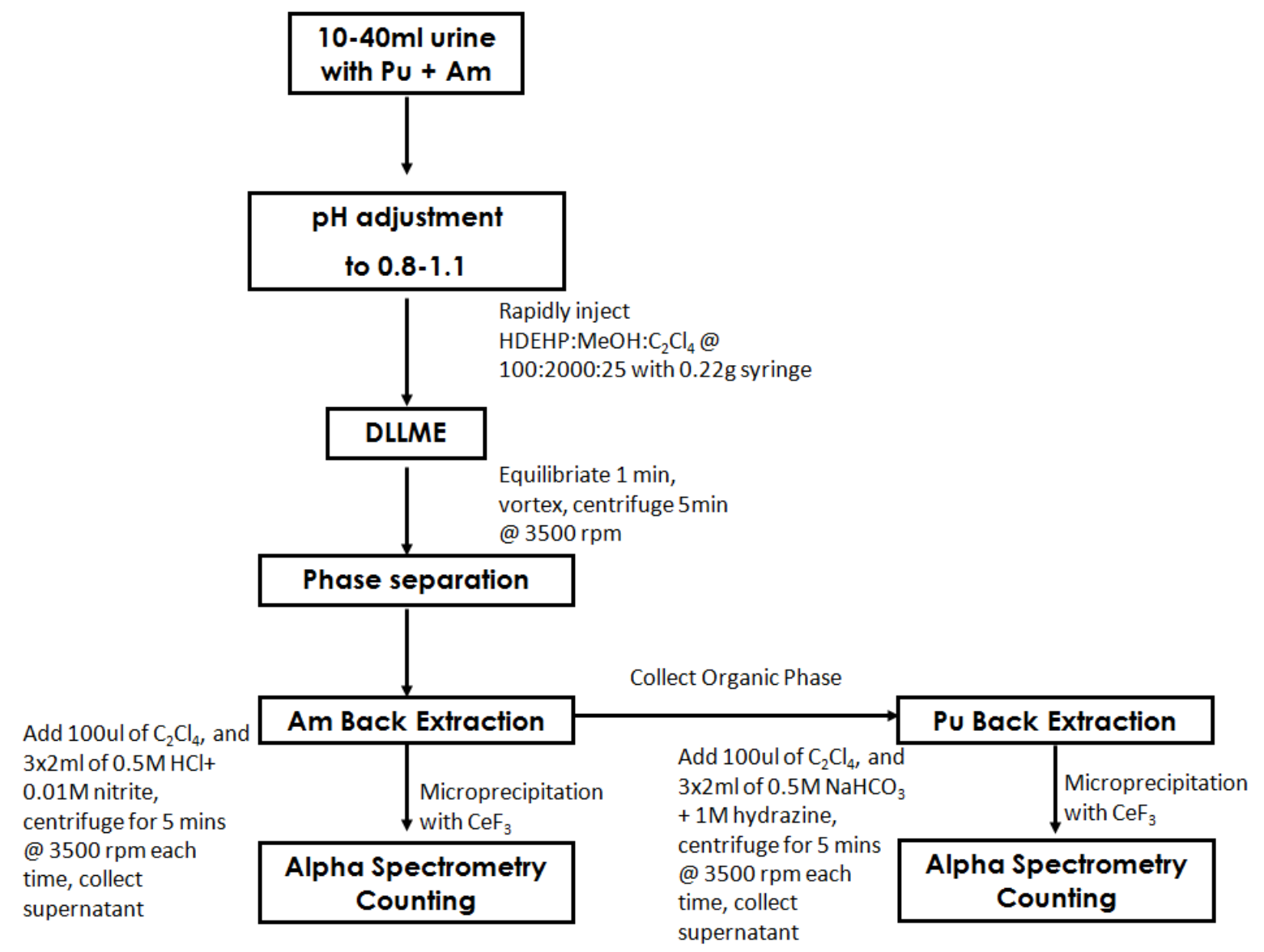

Figure 26: Flowchart for samples containing both $\mathrm{Am}$ and $\mathrm{Pu}$. Turnaround time $=1.25$ hours for preparation + scenario based counting time.

\section{$\underline{\text { 3.17 Minimum detectable activity }}$}

The minimum detectable activity was calculated using Currie's equation [34]:

\section{Equation 7}

$$
M D A=\frac{2.71+4.65 \sqrt{B}}{E \times T \times P}
$$

Where

$\mathrm{B}=$ average total counts in the blank from 5 replicates

$\mathrm{E}=$ combined efficiency $\mathrm{C} 1 * \mathrm{C} 2$, where $\mathrm{C} 1$ is average chemical recovery, and $\mathrm{C} 2$ is the instrument counting efficiency for alphas

$\mathrm{T}=$ total count time

$\mathrm{P}=$ probability or branch ratio for an alpha emission 
The MDA on the Tri-Carb $3180 \mathrm{TR} / \mathrm{SL}$ for the current DLLME method works out to be $0.03 \mathrm{~Bq} /$ sample, using $\mathrm{B}=103, \mathrm{C} 1=0.84$ from replicate samples, and $\mathrm{C} 2=1$ for alphas. The count time was the $1800 \mathrm{~s}$, and $\mathrm{P}=1$ since all alphas are observed.

The MDA on the Ortec Octete for the current DLLME method works out to be 0.42 $\mathrm{mBq} /$ sample for $\mathrm{Pu}-238$, using $\mathrm{B}=11, \mathrm{C} 1=0.82$ from replicate samples, and $\mathrm{C} 2=$ $25.3 \%$ for alphas and $\mathrm{P}=0.709$. The count time was $72000 \mathrm{~s}$.

The MDA on the Ortec Octete for the current DLLME method works out to be 0.42 $\mathrm{mBq} /$ sample for $\mathrm{Pu}-239$, using $\mathrm{B}=10, \mathrm{C} 1=0.82$ from replicate samples, and $\mathrm{C} 2=$ $25.3 \%$ for alphas and $\mathrm{P}=0.708$. The count time was $72000 \mathrm{~s}$.

\section{Conclusions}

A DLLME method for plutonium in urine was developed using methanol as a disperser solvent, tetrachloroethylene as an extractant solvent, and HDEHP as the extractant. It was shown that salt concentration, sonication, centrifugation time, and nitrite did not improve the recovery of plutonium.

For emergency screening, the DLLME method is able to extract all isotopes of plutonium and americium at once and it will be a rapid way to determine if a person is contaminated with any type of alpha emitter. Traditionally, emergency urine samples are screened for the presence or absence of radiation by directly mixing a small volume with the scintillation cocktail, and the MDA is determined by the sensitivity of the instrument. Using DLLME offers an additional preconcentration factor, resulting in a lower MDA. Based on a conservative $0.1 \mathrm{~Sv}$ annual effective dose limit for emergency situations from 
the International Commission on Radiation Protection (Special Guidance \#4), Li et al [35] derived an $\mathrm{Pu}-238$ activity in urine to be $8.2 \mathrm{mBq}$ per $1.6 \mathrm{~L}$ of urine, collected 2 days after exposure, to be indicative of a dose of $0.1 \mathrm{~Sv}$. This works out to be $0.51 \mathrm{mBq}$ per $100 \mathrm{ml}$ of urine, and the DLLME method utilizing alpha spectroscopy with a count time of $72000 \mathrm{~s}$ meets these requirements. Other literature methods for $\mathrm{Pu}-238$ have comparable MDAs [36, 37]

Back-extractability of plutonium and americium from the organic phase allows for low level measurement by alpha spectrometry. However, there is some plutonium (5$10 \%$ ) that is washed out with the americium, and if the plutonium isotope is 238 , a more thorough separation is required because they are not separable by their alpha energies.

This extraction has also shown versatility in being able to analyze DTPA-chelated plutonium and also the high salt fraction of calcium phosphate precipitated plutonium.

Further work can be done on the separation of plutonium from uranium either by finding ideal back extraction conditions from HDEHP or by selective extraction of uranium first. There are also currently no certified reference materials (CRM) for plutonium in urine, and it is possible plutonium that has been metabolized behaves differently in the extraction process, so it will useful to test it, to see how well the method holds up. Finally, the small volume of organic phase produce after extraction is very compatible with field deployable liquid scintillation counters such as the Triathler (Hidex), which require small sample vials. 


\section{Part B: Determination of Americium 241 distribution in bone samples by extraction chromatography and alpha spectroscopy}

\section{Introduction}

The health risks of an internalized radionuclide are related to many factors including the type of radiation it emits, its activity, its chemical form, and the route of exposure. Radiation dose from an intake of an internalized radionuclide can be calculated using biokinetic and dosimetric models (Fig 27) as well as radiation weighting factors (Table 11) and tissue weighting factors (Table 12) recommended by the International Commission on Radiation Protection (ICRP) [38].

Table 11: Radiation weighting factor $\mathrm{Wr}$

$\begin{array}{lc}\text { Radiation type } & \text { Radiation weighting factor } \mathbf{W}_{\mathbf{R}} \\ \text { Photons, all energies } & 1 \\ \text { Electrons, muons, all energies } & 1 \\ \text { Protons and charged pions } & 2 \\ \text { Alpha particles, fission fragments, } & 20 \\ \text { heavy ions } & \text { A continuous function of neutron energy } \\ \text { Neutrons } & \end{array}$

Table 12: Tissue weighting factor $\mathrm{Wt}$

\begin{tabular}{|c|c|c|}
\hline Tissue & $\begin{array}{c}\text { Tissue weighting factor } \\
\text { wT }\end{array}$ & $\Sigma$ wT \\
\hline $\begin{array}{l}\text { Bone-marrow (red), } \\
\text { Colon, Lung, Stomach, } \\
\text { Breast, Remainder } \\
\text { tissues* } \\
\text { Gonads } \\
\text { Bladder, Oesophagus, } \\
\text { Liver, Thyroid } \\
\text { Bone surface, Brain, } \\
\text { Salivary glands, Skin }\end{array}$ & $\begin{array}{l}0.12 \\
0.08 \\
0.04 \\
0.01 \\
\text { Total }\end{array}$ & $\begin{array}{l}0.08 \\
0.16 \\
0.04 \\
1.00\end{array}$ \\
\hline
\end{tabular}




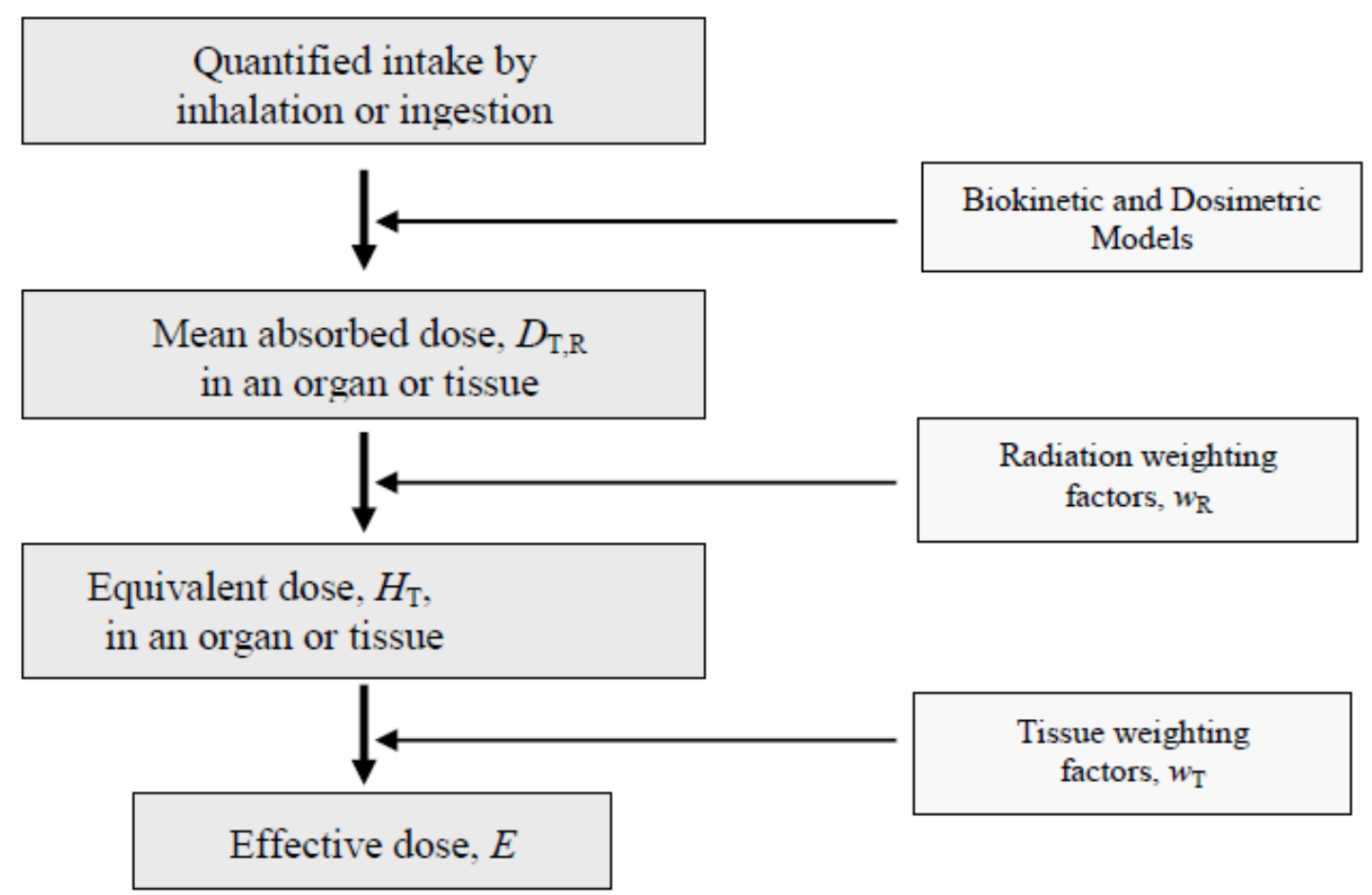

Figure 27: Calculation route from intake to dose [38].

One of the most complicated portions of Figure 27 is the study of biokinetic models, including translating experimental results into mathematical models. Earlier models treated bone as a single compartment which would absorb $45 \%$ of the activity of a bone-surface seeking alpha emitters like plutonium or americium and half of that activity will be released in 100 years (Figure 28). Later on, animal experiments were performed that looked more closely at the bones of rats exposed to actinides to see how actinides are incorporated during growth. It was shown that initial deposition was present on endosteal and periosteal bone surfaces and Am-241 was additionally deposited on vascular canal surfaces. Pu and Am showed transfer to bone volume over the lifetime of the mouse but activity in central marrow was cleared by 448 days [40]. These experiments eventually led to a newer model that takes into the account bone growth and bone turnover (Figure 29). It has also been seen that surface seekers can suppress bone 
resorption by osteoclasts which will slow down the rate at which these surface seekers become buried into the bone volume [41].

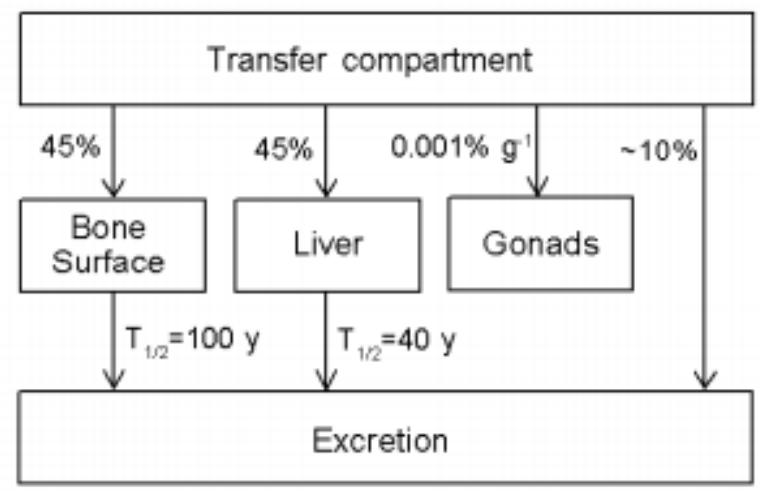

Figure 28: Compartment model from ICRP 30 [39]

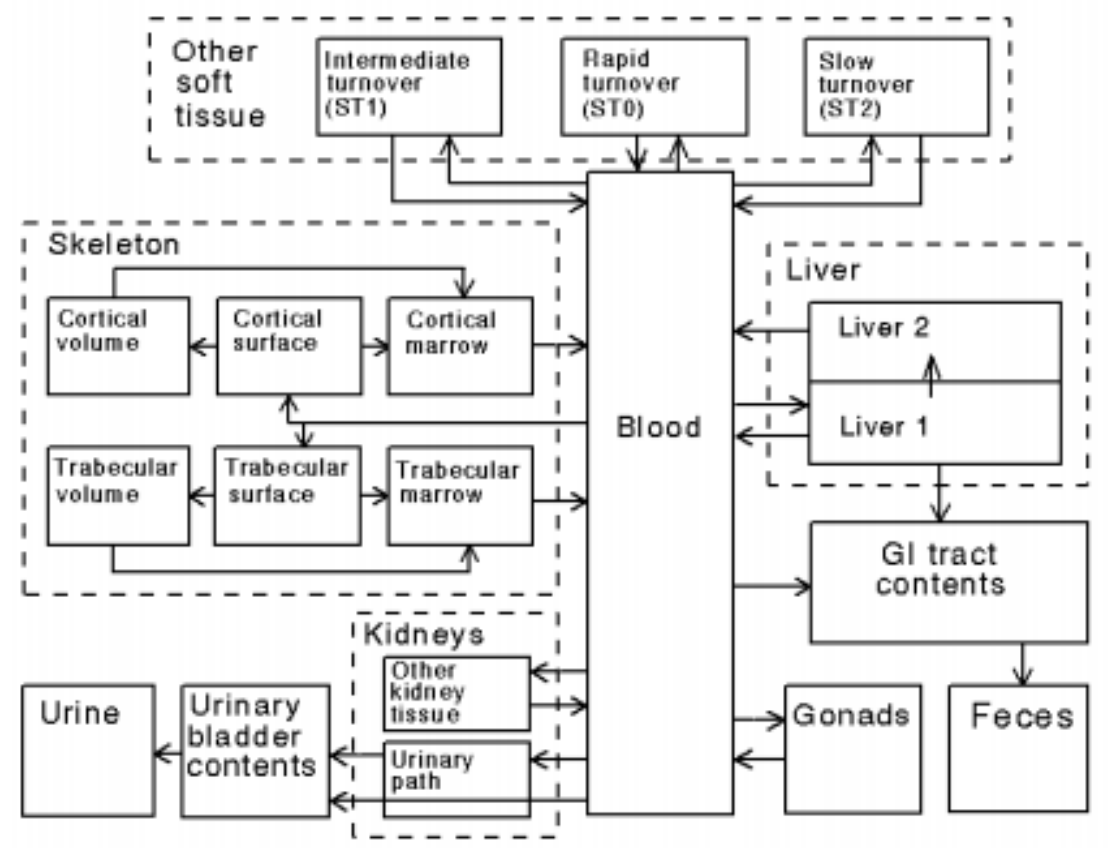

Figure 29: Compartment model from ICRP 103 [39]

\subsection{Chemistry of Americium-241}


Am-241 is formed via beta decay of $\mathrm{Pu}-241$, which is formed from two neutron captures on Pu-239.(Equation 8 ).

\section{Equation 8}

$$
{ }^{239} \mathrm{Pu}(\mathrm{n}, \gamma){ }^{240} \mathrm{Pu}(\mathrm{n}, \gamma){ }^{241} \mathrm{Pu} \stackrel{-\beta^{-}}{\longrightarrow}{ }^{241} \mathrm{Am}
$$

Americium's electronic configuration is $[\mathrm{Rn}] 5 \mathrm{f} 7 \mathrm{7s} 2$ and it can exist in the +3 ,

+5 , or +6 valence states as $\mathrm{Am}^{3+}, \mathrm{AmO}_{2}{ }^{+}$, or $\mathrm{AmO}_{2}{ }^{2+}$, respectively. The $3+$ valence state is the most common and the most stable state. Because it is trivalent, americium follows the same precipitation reactions as the lanthanides and forms insoluble hydroxides, fluorides, and oxalates [8].

Am-243 has produced artificially through Equation 8 and a half -lives and alpha decay energies are presented in Table 1.

\subsection{Previous case of contamination}

In 1976, a worker was extensively contaminated with Am-241 at the Hanford waste management site after an ion-exchange column exploded and sprayed him with radioactive acids and debris. He was treated by DTPA chelation therapy immediately and lived another 11 years before dying of heart disease. His skeletal body burden was $500 \mathrm{kBq}$ in Am-241 at time of death and his body was donated to the United States Transuranium and Uranium Registry (USTUR). The USTUR was established in 1968 with the goal of collecting and archiving tissues from human exposed to internal radiation contamination. Measurements of the activities in these tissues along with records of the exposure scenarios have aided the development and refinement of biokinetic models. 
Slices of bones were created for the purposes of histology and autoradiography. Histological indicators showed bone cell turnover was suppressed. Autoradiographic studies showed bone turnover to incorporate Am-241 within bone but surface americium was still present and a few percentage was found in bone marrow [42].

\subsection{Current case}

The exposed individual, case USTUR 0846, inhaled $\sim 67 \mathrm{kBq}$ of Am-241 during a purification process in 1967 and promptly underwent chelation therapy with DTPA for a course of 7 years with weekly injections. He died in 2008 from pancreatic cancer and it was estimated that his skeleton still contained $30 \mathrm{kBq}$ of Am-241 at the time of death, based on current models. Slices of the humerus were retrieved from USTUR for analysis in this study.

\subsection{Alpha track analysis}

The most accurate method of determining where americium is localized within a bone cross section is by alpha track analysis. This method places the radioactive sample in close contact with a film material which is susceptible to damage from high energy alphas, such as cellulose nitrate or CR-39 (polyallyl diglycol carbonate). Each alpha decay captured by the detector produces a singular track which can be etched using base and viewed under a microscope. The location and density of tracks on the film correspond directly with the spatial distribution of activity in the sample. [43]

\subsection{Bone physiology}

Bones in the human body are classified based on their shape with most bones in the arms and legs considered to be long bones. Long bones are comprised of a narrow 
midsection (diaphysis) containing compact bone with a bone marrow containing central cavity (medullary) and wider ends (epiphysis) near the joints containing spongy bone. The other layer of all bones is covered with a membrane layer known as the periosteum, which contains blood vessels to provide nutrients to the bone [44].

The role of osteoblasts is to produce a strong fibrous protein, collagen, along with depositions of hydroxyapatite (a calcium phosphate mineral) to form the organic bone matrix. Osteoclasts are multinucleated cells containing collagenase secreting vesicles to recycle or resorb old bone. Osteocytes are mature bone cells that no longer divide and are trapped in spaces called lacunae as bone is formed.

In compact bone, osteons are packed layers of bone cells with a central Haversian canal when blood vessels and nerves are located. In contrast, spongy bone has the appearance of a sponge with thin layers of bone (trabeculae) containing osteocytes within these layers. As there is no central canal, blood is delivered to all the spaces between trabeculae.

Bone growth at the epiphysis happens when cartilage at the epiphyseal plate facing the diaphysis is calcified for osteoblasts to start forming new bone. On the epiphyseal plate facing the joint, new cartilage is formed and it is this gradual movement that causes elongation formation of long bones.

Bones are the main storage reservoir for calcium in the body, and when the body requires calcium for signal transduction, clotting, or enzyme activity, parathyroid hormone (PTH) stimulates the activity of osteoclasts to break up bone in order to raise serum calcium levels. During this process, metals like americium which had been deposited in the bone will also be released back into the blood. The rate at which this 
happens over a lifespan will depend on the person's diet, level of exercise, and medical condition. [46]

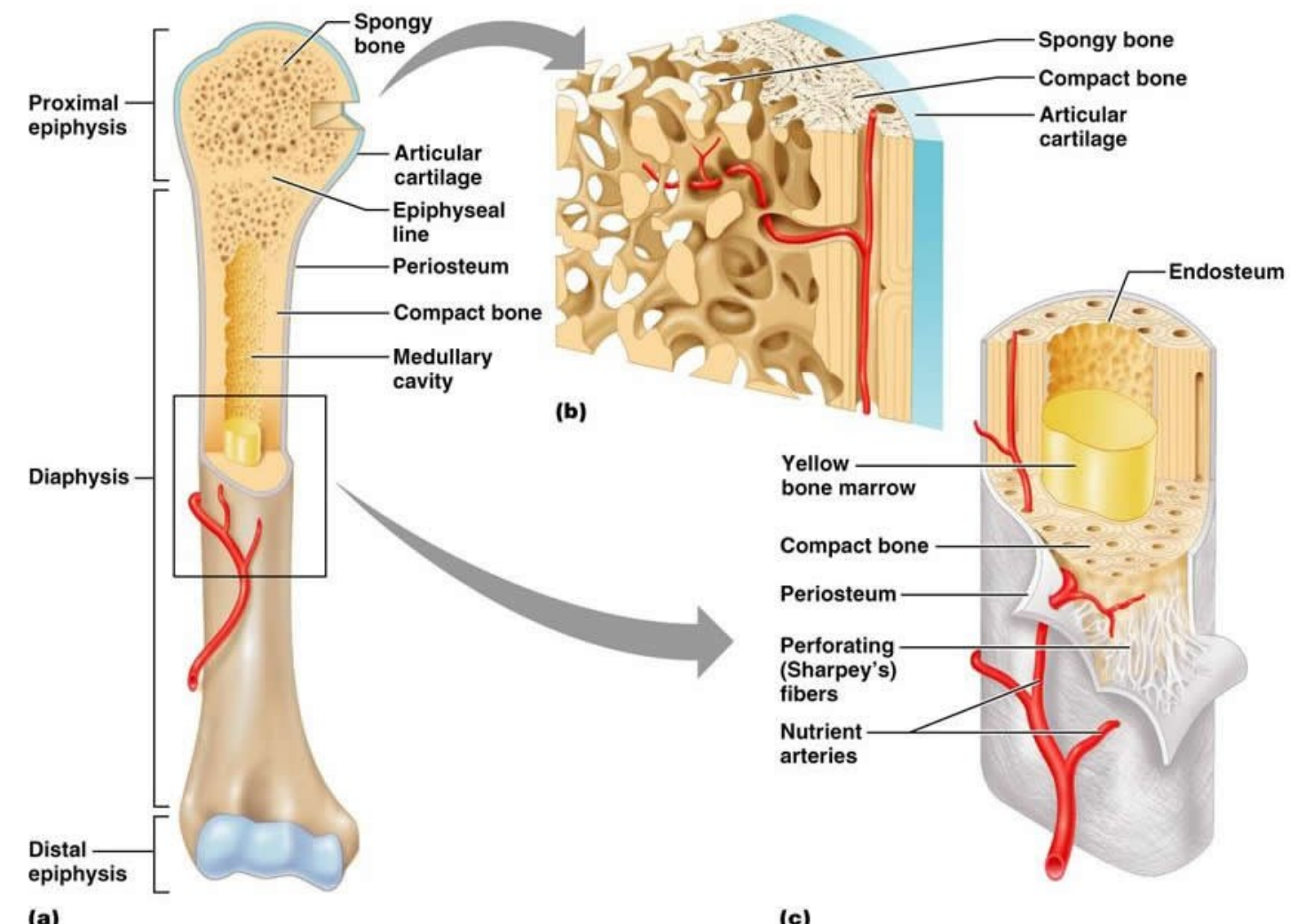

(a)

(c)

Figure 30: The structure of long bone [45].

\subsection{Models}

Current models and biokinetic coefficients used in dose assessment assume the deposition of $\mathrm{Am}$ (and also $\mathrm{Pu}$ ) on bone surfaces, with the activity and rate of deposition dependent on route of intake, and the radionuclide's physio-chemical properties.

Any americium deposition on the bone has to first come from contact with the blood stream, therefore, the highest initial deposition is on endosteal surfaces of trabecular bone which has a high surface area to mass ratio and is surround by blood. 
There will also be some deposition on the periosteal surface and near the vascular channels of the cortical bone by the same rationale. [47]

Bone remodeling over time decreases $\mathrm{Pu}$ and $\mathrm{Am}$ on trabecular surfaces and lowers dose to cells and even though initial uptake inside of cortical bone is minimal or absent. Animal studies have shown remodeling occurs twice as fast in vertebrae as in the distal bone highest concentrations found in baboon were in vertebrae, sternum and less in long bones [48].

\subsection{Sample processing}

Sample processing consists of four steps: bone sectioning, ashing, chemical separation, and counting. Chemical separation is performed to remove traces of uranium, radium, and calcium from the samples which can otherwise interfere spectrally with americium determination or be physically incorporated into the final precipitate used for alpha counting. As described in Part A section 3.12 having increase bulk in the precipitate will decrease alpha efficiency and resolution.

\subsubsection{Extraction chromatography}

In extraction chromatography, liquid extractants are sorbed on the surface of an inert support material and the mobile phase containing the analyte of interest is passed through and retained by the extractant. The high surface area for the extractant to interact with the analyte results in high distribution ratios. An ideal extractant should have high specificity, high capacity, and high retention during loading, and low retention during elution. 
Diglycolamides have been investigated since the 1990s and have shown preferential affinity for trivalent metal ions. It was rationalized using the hard \& soft acid base (HSAB) concept that interaction with the metal ion was mainly with the hard oxygen donors and the nitrogens do not take part in complexation [49]. The hydrophobicity of diglycolamides is directly related to the length of its constituent alkyl groups. It was seen that alkyl groups below butyl were soluble in water while octyl chains and higher were not. However, the separation factor also decreased with alkyl chain length because of steric hindrance between adjacent branches preventing efficient complex formation with the metal ion. $\mathrm{C} 8$ was found to be the optimal alkyl chain length for both extraction and aqueous immiscibility [50].

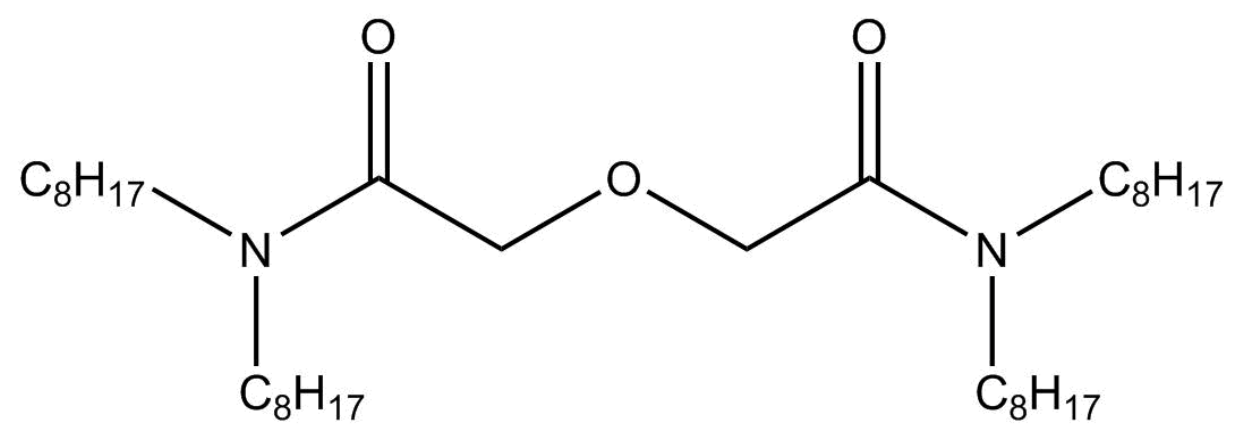

Figure 31: Chemical structure of DGA

In the 1990s, Horwitz et al. supported $\mathrm{N}, \mathrm{N}, \mathrm{N}^{\prime}, \mathrm{N}^{\prime}-$ Tetraoctyl Diglycolamide (TODGA or DGA for short) onto Amberchrom CG71 polystyrene resin (Fig.34). By attaching the extractant to an immobile phase it minimizes carryover of the extractant to the aqueous phase. Another advantage of extraction chromatography is that high matrix concentration of calcium should not interfere with selective columns where as it can with ion exchange chromatography. Equation (9) shows the interaction of americium with DGA 


\section{Equation 9}

$$
\mathrm{Am}^{3+}+3 \mathrm{DGA}+3 \mathrm{NO}_{3}^{-}->\mathrm{Am}(\mathrm{DGA})_{3}\left(\mathrm{NO}_{3}\right)_{3}
$$

In the past decade, separation of americium on DGA supported columns have become well accepted in the radiochemistry community and applications to matrices such as vegetation [51], air filters[52], and emergency bioassay[53]. Many of these methods use dilute $\mathrm{HCl}$ to elute Americium since its k' factor (similar to D from equation 1 where a higher k' represents a greater fraction of the metal on the stationary phase) is orders of magnitude lower than in high nitric acid concentrations [54, 55]. Figures 35 and 36 show the retention of americium (middle) on DGA in $\mathrm{HNO}_{3}$ and $\mathrm{HCl}$ media.

\subsubsection{Ashing procedures}

A published process on bone ashing first dries sample in a drying oven at $110^{\prime} \mathrm{C}$ for several days. Afterward, the bone sample is transferred to a muffle furnace and ramped between $150-450$ ' $\mathrm{C}$ for $8-12$ hours while maintaining $450^{\prime} \mathrm{C}$ for another 24 hours. Then the sample is wet ashed using $8 \mathrm{M} \mathrm{HNO}_{3}$ until all visible carbonized material was destroyed [56] 

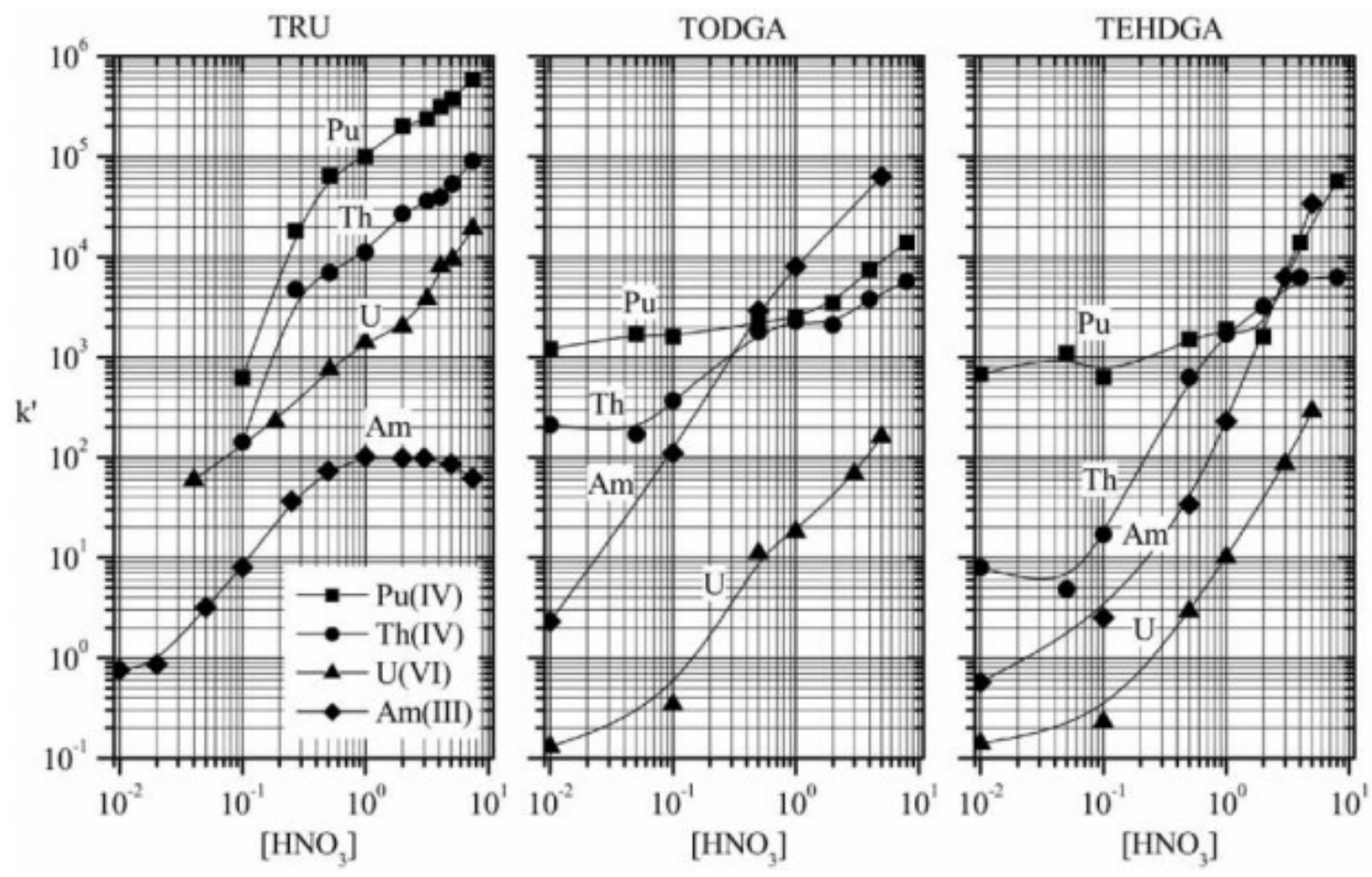

Figure 32: Relationship between retention and $\left[\mathrm{HNO}_{3}\right]$
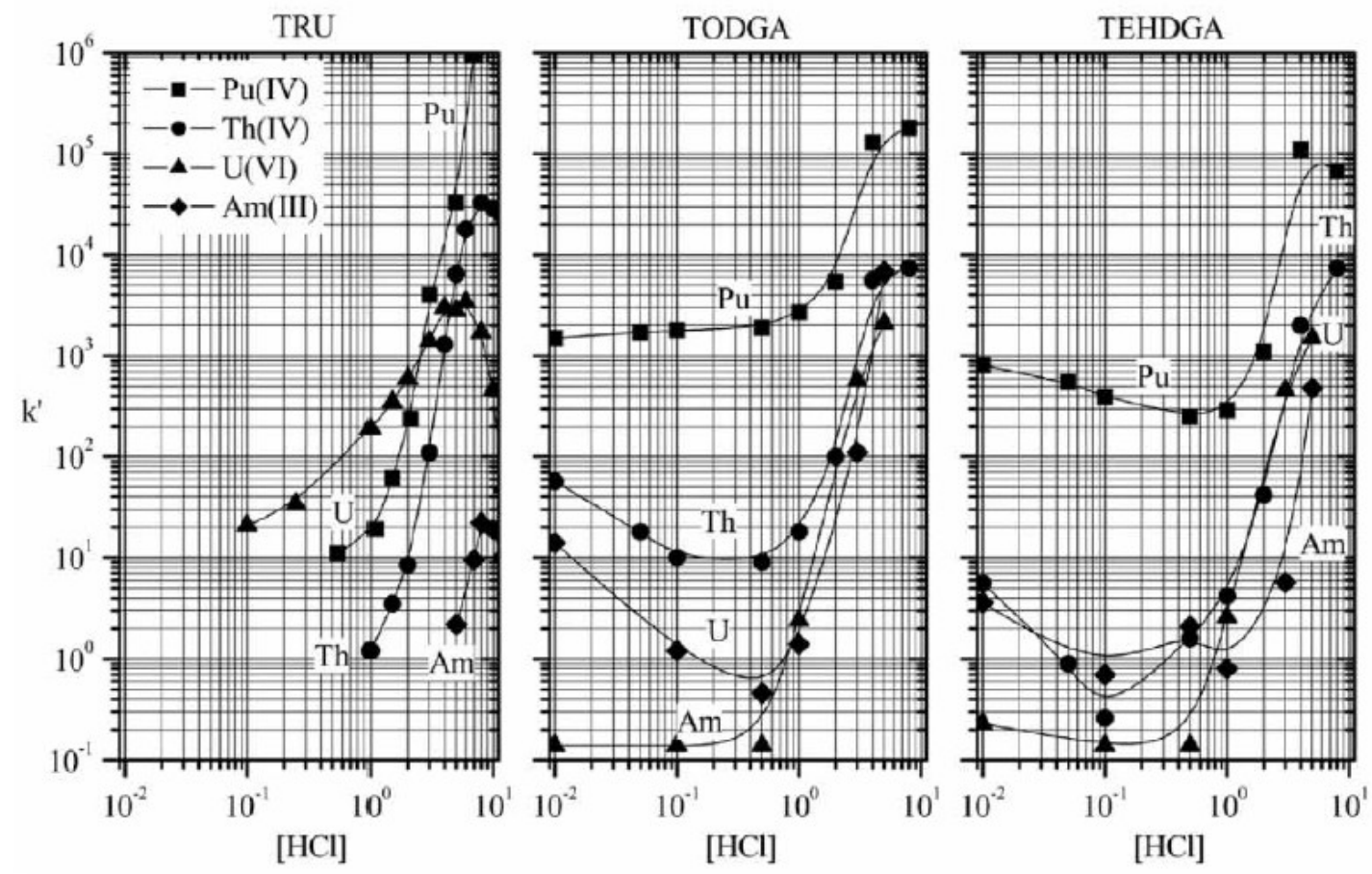

Figure 33: Relationship between retention and [ $\mathrm{HCl}]$ 


\subsection{Scope}

Objective of study:

1) To test if an alternate method to autoradiography is possible for the determination of americium distribution in a long bone sample, containing low levels of americium

2) To test a previously developed method for the measurement of americium-241 in urine can be applied to bone samples as well

3) To study the amount of americium-241, if any, is incorporated into the bone volume, decades after an acute exposure

\section{Materials and Methods}

\section{Reagents and supplies}

All dilute solutions were prepared using distilled water filtered through a Millipore filtration system $(16 \mathrm{M} \Omega / \mathrm{cm})$. The mix-grafter tool was purchased from Salvin Dental Specialties (USA). Quartz crucibles were purchased from Advaluetech (USA). Concentrated nitric acid and hydrochloric acid were of ACS grade (Fisher Scientific, Canada). Sodium nitrite was of trace metal grade (Sigma-Aldrich, Canada). DGA resin cartridges (100-150um particle size, 2ml BV) and empty adapter cartridges were purchased from Eichrom (USA). Filter discs and funnels were purchased from Eichrom (USA) and mounting adhesives were purchased from Soquelec (Canada).

Am-243 is NIST-certified and purchased from Eckert and Ziegler (USA). Am241 used for method validation was purchased from Amersham (UK).

\subsection{Sampling}


Based on USTUR case 0246, using a bone from a person contaminated with a skeletal burden of $500 \mathrm{kBq}$ of Am-241 and requiring up to $0.5-3$ years of development time on radiographic film, the bone from our current case study will require over 10 years of development time. To provide a quicker estimate of where Am-241 was deposited (cortical vs trabecular) after 40 years of bone turnover, an alternate method where successive layers of bone were shaved, and measured by alpha spectrometry, was used. The tool used to shave the bone samples was an mx-grafter, which contains a chamber to hold shavings (Fig.34).

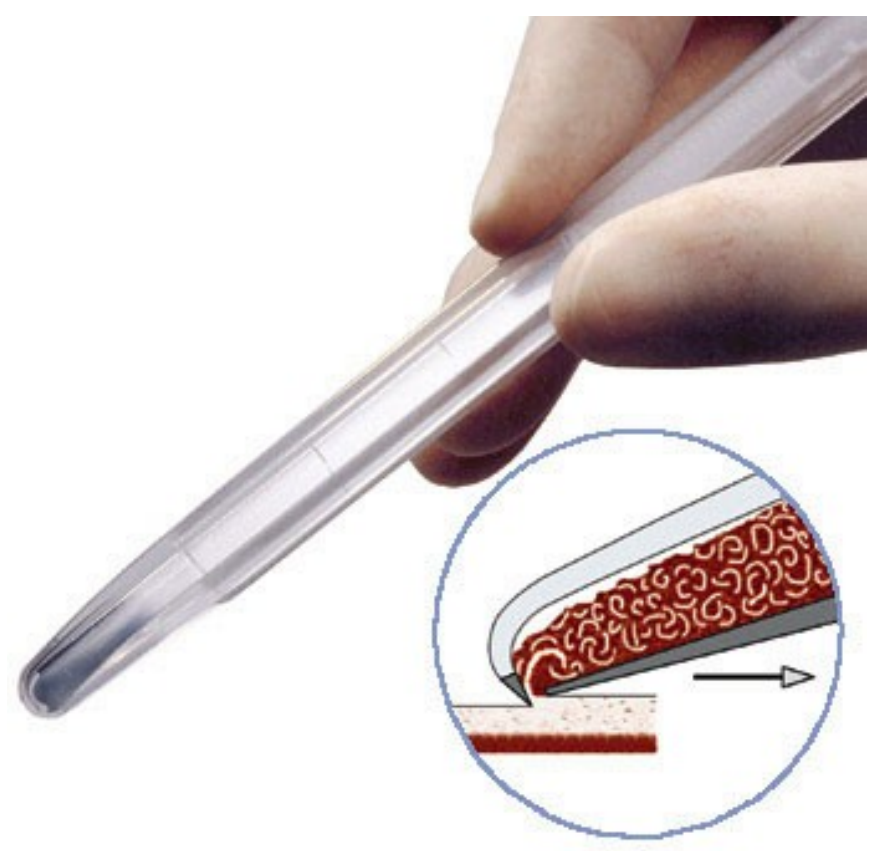

Figure 34: MX grafter tool, with chamber shown in inset

The bone selected was the left humerus and two slices were obtained. The first slice was closer to the distal shaft of the bone and contained marrow, cortical, and trabecular bone. The second slice was obtained from the middle of the humerus and contained nearly all cortical bone. 
The slice of bone was marked with a line to differentiate the cortical bone from the trabecular bone. The cortical bone was divided into three sublayers: a) outer surface to include periosteum, b) middle cortical containing osteons, and c) cortical-trabecular bone interface.

each bone sample was first measured with a caliper and photographed in the upright position before cutting. The bone was then sampled using the mx-grafter tool with the addition of $\mathrm{mQ}$ water to wet the sample and the bone was scaped along the longitudinal axis of the bone. After sampling one layer, the chamber of the mx-grafter was emptied into a quartz crucible, and the bone has measured again with a caliper and photographed.

\subsection{Radiochemistry}

After sampling, the bone shavings were placed in a drying oven for 2 hours at $120^{\prime} \mathrm{C}$ to evaporate off the water. The mass of the bone after this process is referred to as the dry mass. Am-243 tracer was added. The bone was then dry ashed in a muffle furnace using a ramp from room temperature to $600^{\prime} \mathrm{C}$ for 6 hours, and a holding period at $600^{\prime} \mathrm{C}$ for another 6 hours. The sample was cooled and weighed. The mass remaining is referred to as the ashed mass. The bone ash was transferred to a beaker with $15-20 \mathrm{~mL}$ of $1 \mathrm{M} \mathrm{HNO}_{3}$. This was heated to near boiling and $1.5 \mathrm{~mL}$ of conc. $\mathrm{HNO}_{3}$ and $0.5 \mathrm{~mL}$ of $30 \% \mathrm{H}_{2} \mathrm{O}_{2}$ were added to wet ash any insoluble compounds. The solution was evaporated to dryness and re-dissolved in $15-20 \mathrm{~mL}$ of $5 \mathrm{M} \mathrm{HNO}_{3}$. Am separation on DGA was then performed using previously published conditions [49]. Briefly, DGA column was preconditioned with $10 \mathrm{~mL}$ of $5 \mathrm{M} \mathrm{HNO}_{3}$ and the sample added. An additional $15 \mathrm{~mL}$ of $5 \mathrm{M} \mathrm{HNO}_{3}$ was used to rinse out the beaker and load to the column. Interferences were eluted with $15 \mathrm{~mL}$ 
$0.1 \mathrm{M} \mathrm{HNO}_{3}+0.03 \mathrm{M} \mathrm{NaNO}_{2}$. Finally, americium was eluted from the DGA with $10 \mathrm{~mL}$ of $0.1 \mathrm{M} \mathrm{HCl}+0.03 \mathrm{M} \mathrm{NaNO}_{2}$.

\subsection{Alpha Spectroscopy}

Alpha spectrometry counting was performed on an 8 chamber Ortec Octete spectrometry system fitted with $450 \mathrm{~mm}^{2}$ Canberra PIPS detectors.

\section{Calculations}

The recovery of the Am-243 tracer was determined using Equation 10.

\section{Equation 10}

$$
\% \text { Rec. } 243=100 \times \frac{\frac{243 c}{t e}}{243 k}
$$

Where

$243 \mathrm{c}=\#$ counts for the Am 243 peak

$\mathrm{t}=$ total count time for the sample

$\mathrm{e}=$ combined counting efficiency and chemical recovery

$243 \mathrm{k}=$ known activity of tracer based on mass added

Then the activity of Am241 was calculated using Equation 11.

\section{Equation 11}

$$
A m 241=\frac{\frac{241 c}{t e}}{\text { Rec. Am243 }}
$$

Where

241c $=\#$ counts for Am-241 peak

$\mathrm{t}=$ counting time

$\mathrm{e}=$ combined counting efficiency and chemical recovery

Rec.Am243 = recovery of Am243 tracer 


\section{$\underline{3 \text { Results and Discussion }}$}

\subsection{Alpha spectrum deconvolution}

Am-241 in the bone was calculated based on the recovery of the tracer, Am-243. The energies of these two isotopes are only $200 \mathrm{KeV}$ apart. Many of the samples had poorer resolution in the sample compared to the DLLME experiments and this can be attributed to self-absorption, and interfering precipitates such as CaF2. Even though most divalent metals should have been washed from the column, calcium accounts for $18-22 \%$ of the bone's mass and it is possible that residual calcium is also in the eluent [53]. Another explanation is that the microprecipitate may not have formed a uniform layer on the filter disc. The poorer resolution means some of the counts from the tail of the Am241 peak will overlap with those from the Am-243 and cause an overestimation of Am243.

To correct for this overlap, fitting equations based on alpha spectrum deconvolution was applied to the spectra. Alpha peaks can be modeled as Gaussian distributions with the centroid being at the known energy of alpha decay for a particular isotope. In microprecipitated samples, the alpha particles can be inhibited by the bulk lanthanide precipitate which can cause attenuation and lowers the energy of the alpha reaching the detector. This is modeled by a modified Gaussian function. The alpha spectrums of bone samples containing overlapping Am-241 and Am-243 peaks were fitted with these equations with the constraint that the sum of counts from the fitted peaks is identical to the sum of counts from an unfitted spectrum, in the same region of interest [57] (Fig.35). 


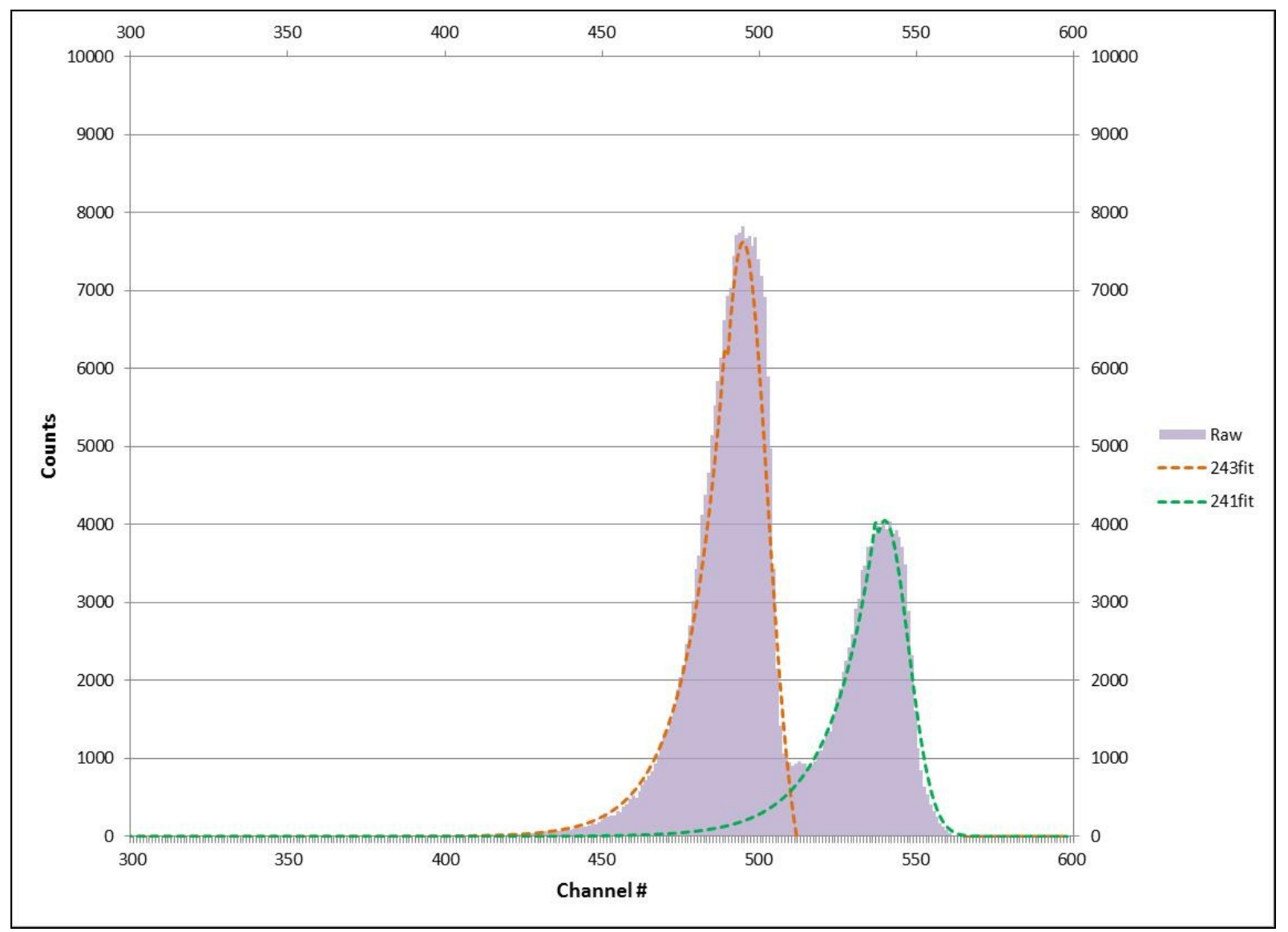

Figure 35: Fitted curves overlaid on raw spectrum.

\subsection{Development of methodology}

A preliminary set of triplicate shavings from a lamb bone was used to determine an appropriate drying time, ashing temperature, and ashing duration. A drying time of 2 hours using a temperature of $120^{\prime} \mathrm{C}$ was deemed sufficient in removing the moisture from each collection of bone shavings since there was no change in mass between the $2^{\text {nd }}$ and $3^{\text {rd }}$ hours. This drying time was much less than the drying times reported for whole intact bone, due to the bone shavings being much thinner. For dry ashing, a bone sample with a chalk-white appearance is considered to be fully ashed. At temperatures below 300 'c, the organic compounds in the bone shaving begin to char but their shape and appearance remain the same. According to previous studies, a temperature of $450^{\prime} \mathrm{C}$ for 12 hours is 
expected to be enough to ash bone. However, two out of the three samples had gray shavings instead of white shavings so $600^{\prime} \mathrm{C}$ for 12 hours was used for the dry ashing conditions. There have also been other reported where bone was ashed at temperatures of $1000^{\prime} \mathrm{C}$, but these reported were not specific for radionuclide or metals analysis.

After dry ashing, the bone ash was dissolved in $8 \mathrm{M} \mathrm{HNO} 3$ and $\mathrm{H} 2 \mathrm{O} 2$, and wet ashed until dryness. Then the residue was redissolved in 5M HNO3. Sample purification and analysis followed the same procedure as in 2.2 Methods, and. the maximum recovery is determined by the efficiency of the alpha system spectrometry which is between 25$30 \%$. The additional losses can be come from the separation process or incomplete microprecipitation. The final recovery of the Am-241 activity was $81 \pm 7 \%$ based on the Am-243 tracer.

\subsection{Americium Distribution}

\subsubsection{Bone sample 1}

Bone sample 1 was a thin slice of bone with half of the bone missing (Figure 36). This piece of bone was used to test the sampling method and there were also regions of visible trabecular bone (dark black and green portions of Figure). Figure 37 shows the specific activity of the ashed bone with each sub-sample. The ashed bone was used because the bone has fibrous membranes both on the periosteum and endosteum that are difficult to isolate from the bone and will also contribute to the mass. Sub-sample 1 was the marrow, which is expected to have very little activity but storage conditions could have leech americium from the trabecular bone back into the marrow. Sub-samples 2 and 3 are trabecular bone and these show the highest activity per gram on bone out of all the sub-samples. 


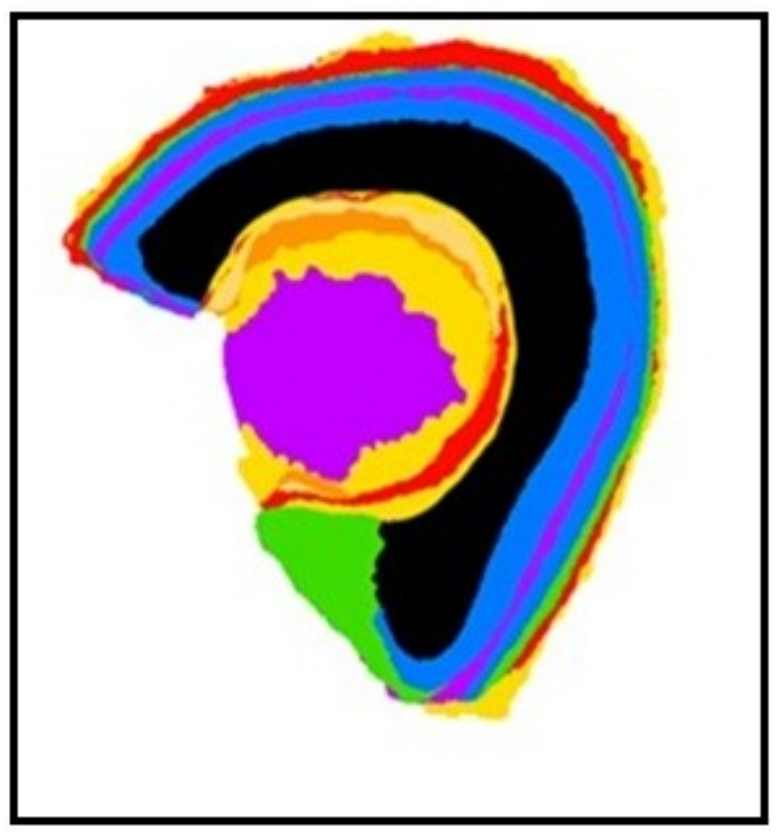

Figure 36: Bone Sample 1

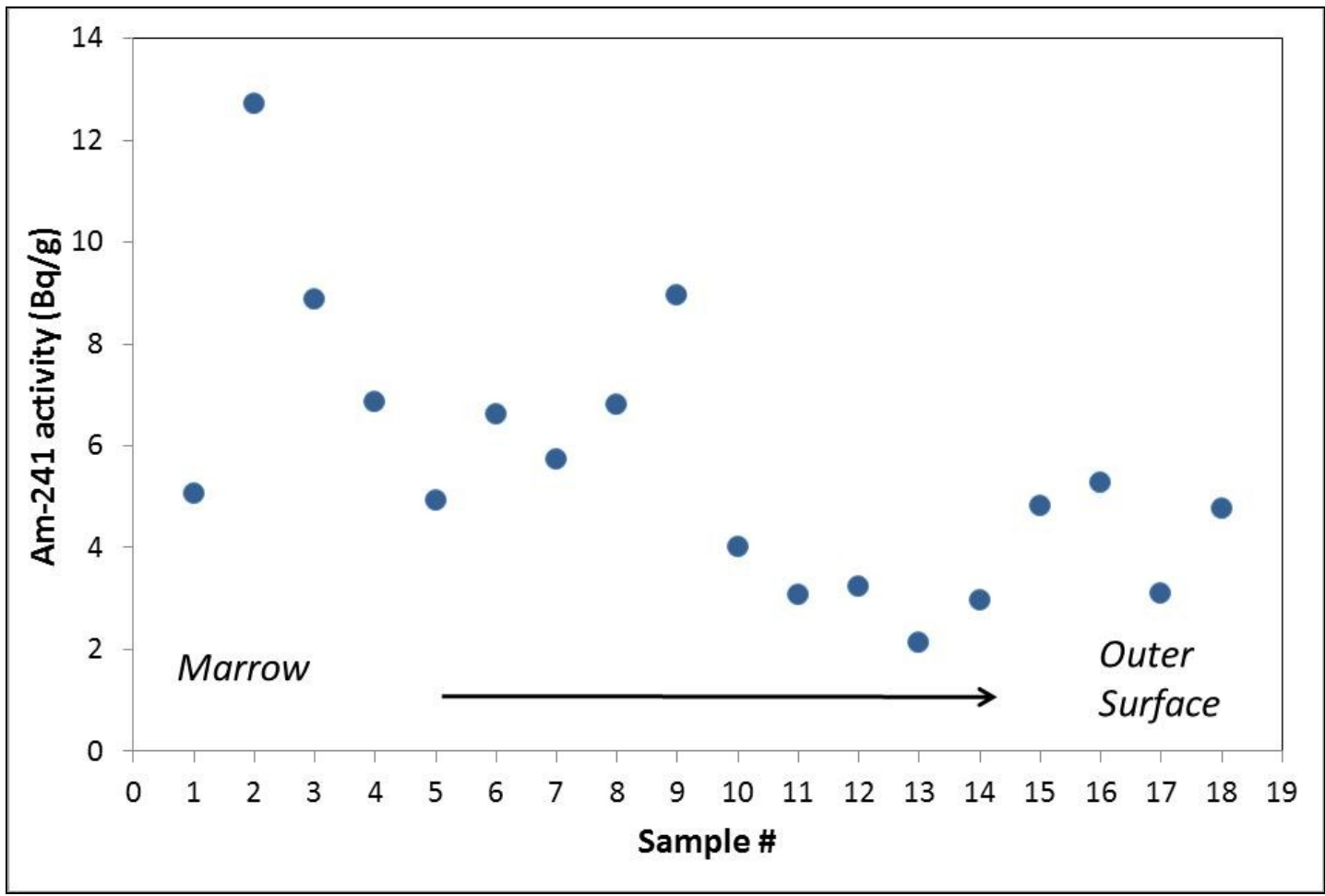

Figure 37: Sub-sample\# vs Am-241 SA in Bone sample 1 Each subsample corresponds to a layer from Figure 36 starting from the marrow. 


\subsubsection{Bone sample 2}

Bone sample 2 was a larger cylindrical bone piece. The photos taken when the bone was shaved were used to estimate the area of bone removed for each sampling. Each end of Bone sample 2 was almost symmetrical, with the exception of a small bone ridge that forms along the ends of one of the bones. For qualitative purposes, the bone was visually divided across the middle with the assumption that there was symmetry between each end of the bone and the middle of the bone. Then photos were analyzed using image processing software GIMP, to determine the number of pixels that were removed. These images were overlaid to give a better idea of the positions of the bone layer.

Figures 38 and 39 show both sides of the bone number correspond to the same sub-sample of bone. The activity in each of these sub-samples is visualized in Figure 40. A similar trend to Bone sample 1 can be seen where the specific activity in the endosteum is higher than the middle cortical. The periosteum on either side also has high activity which can be a result of storage conditions like the marrow in Bone sample 1 or bone turnover is slower on periosteal surface and the activity is not incorporated into the bone. Since bone turnover in trabecular bone is $4 \mathrm{x}$ that of cortical bone (ICRP72b), it is predictable to have more americium incorporated into the bone volume near the trabecular bone. As bone turnover increases as the person ages, the toxic effects of americium are reduced by being incorporated deeper into the cortical bone where less of the alpha energy can reach the developing cells. 


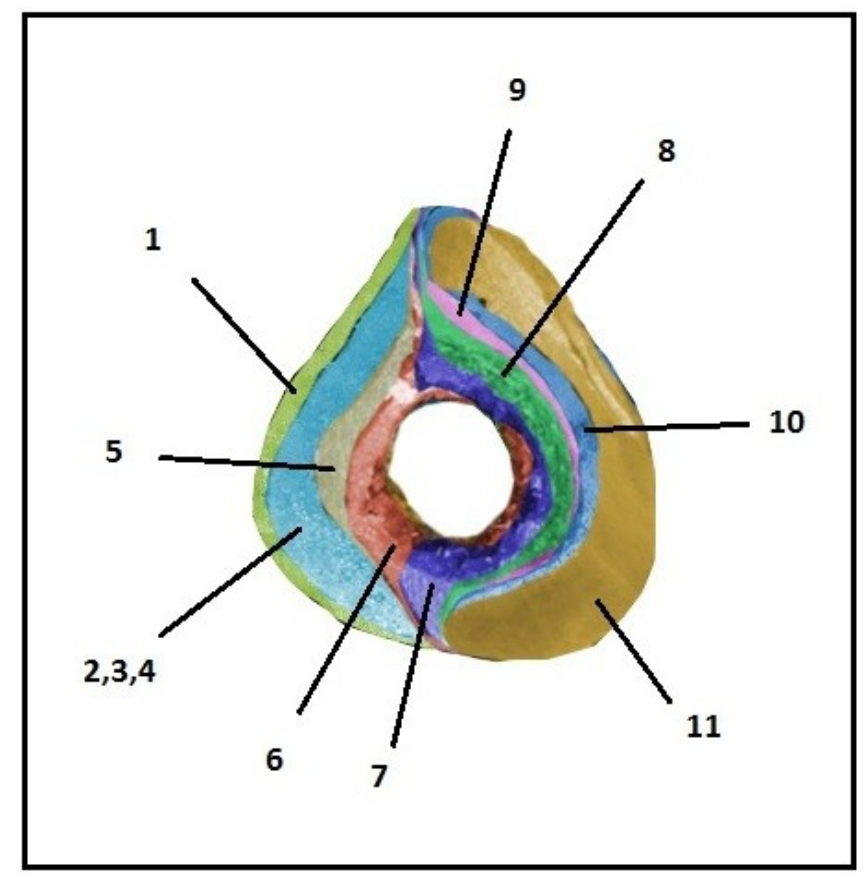

Figure 38: Side of bone sample 1 facing distal end.

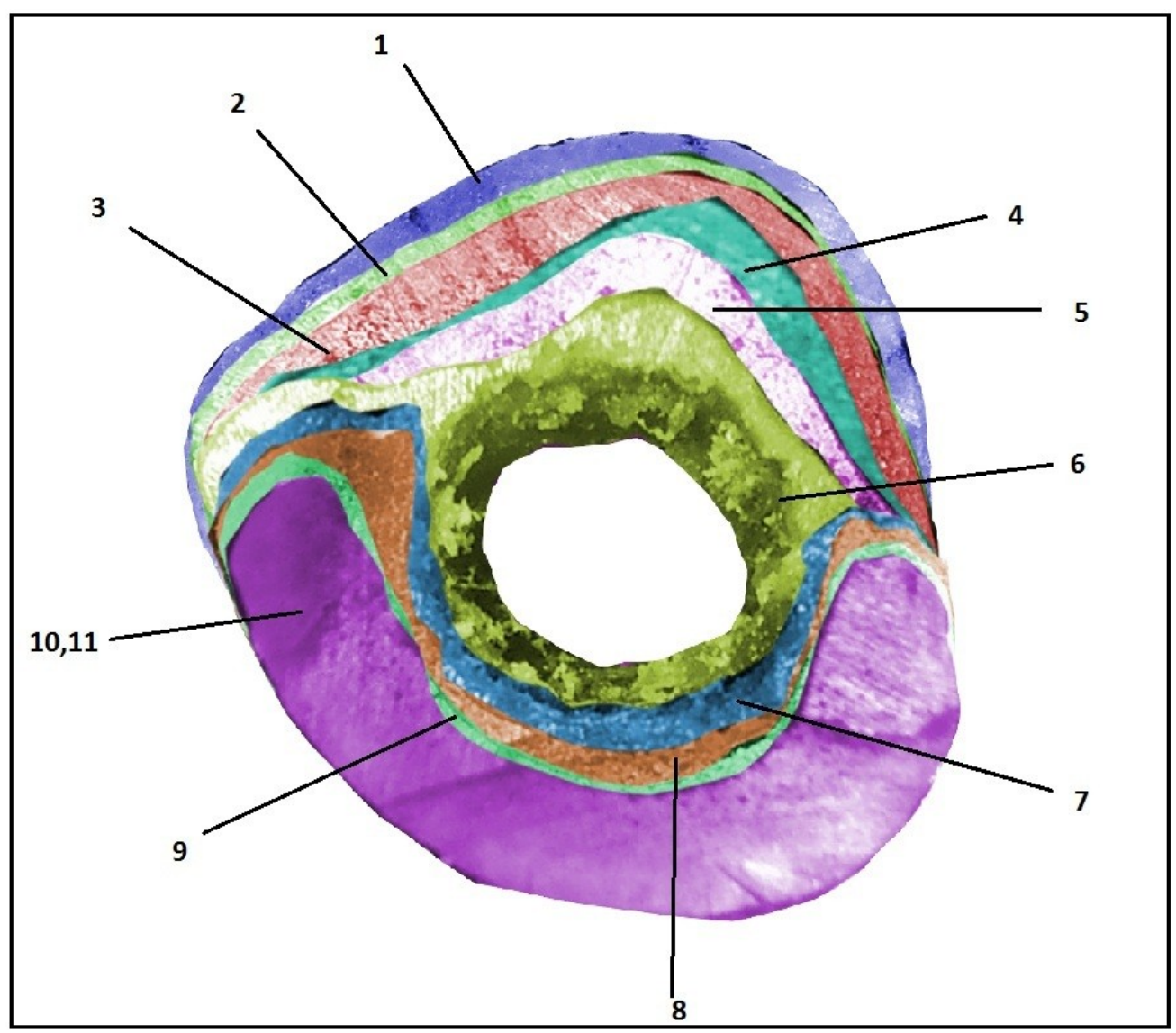

Figure 39: Side of bone sample 2 facing middle 


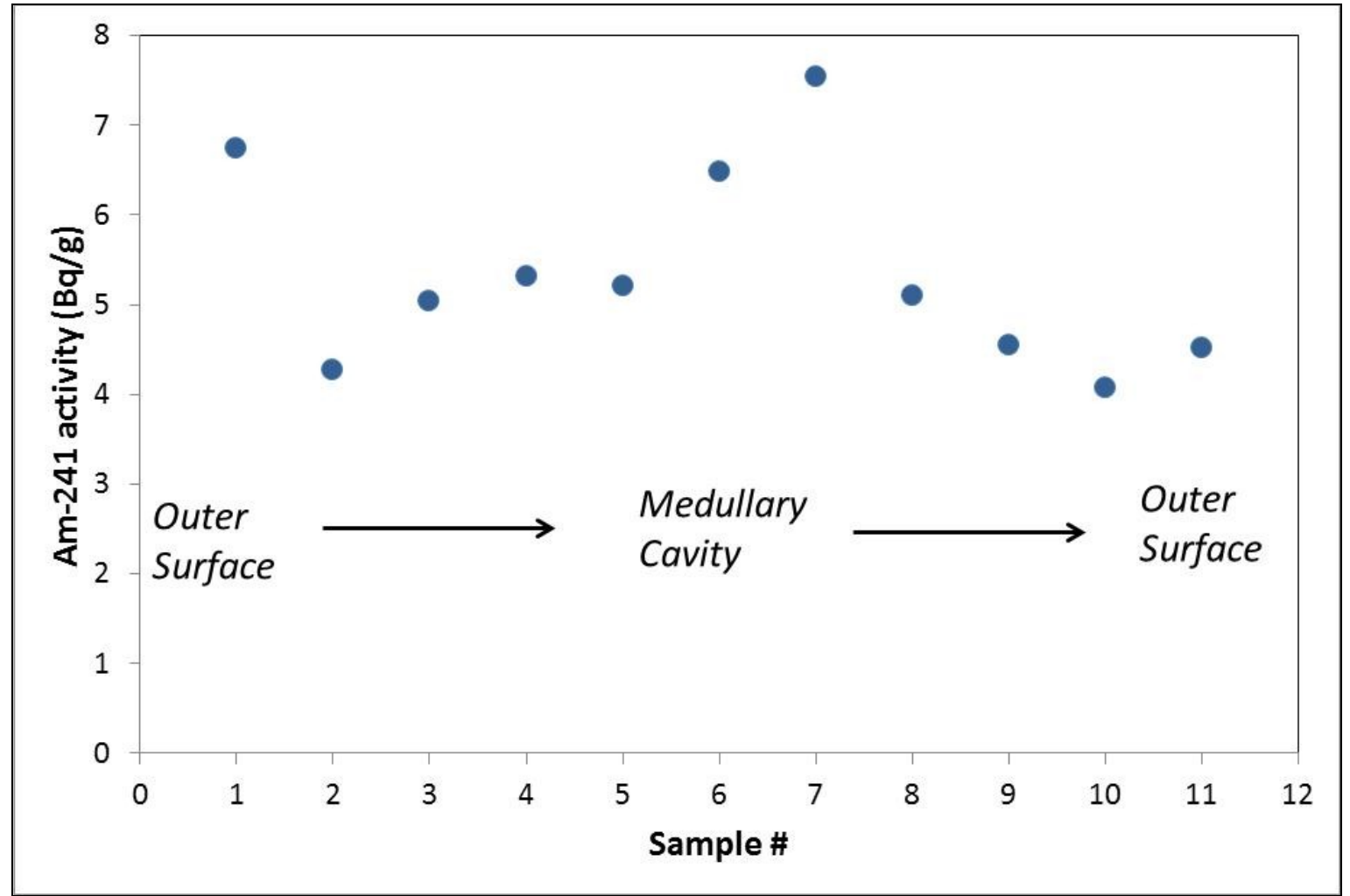

Figure 40: Sub-sample \# vs Am-241 SA in Bone sample 2. Each subsample number corresponds to a layer from Figures 38 and 39.

\section{Conclusions}

The DGA based emergency bioassay method for americium separation was applicable to bone samples after the bone was dry and wet ashed. In cases where there was overlap between Am-241 and the tracer Am-243, a fitted curve, based on spectral deconvolution can be used

Two pieces of bone were shaved using a new sampling method and the endosteal surfaces were found to still contain the highest specific activity of americium. While the sampling method can only provide an estimate of the deposition areas, and uncertainties are difficult to estimate, americium was present in all layers. The activity in both subsamples have an average activity of $4.8 \mathrm{~Bq} / \mathrm{g}$ of ashed bone, and if a reference man 
weight of $70 \mathrm{~kg}$ and the ashed skeleton is assumed to be $7 \%$ of the body mass, then the total body burden is about $23.5 \mathrm{kBq}[58]$. This is a lower than the $30 \mathrm{kBq}$ that was calculated for this case study, but note that both samples were of cortical bone, and trabecular bone would have a higher concentration of Am-241 per gram because of the higher surface area. This means current models developed from animal experiments are quite accurate in estimating long-term americium retention in humans.

It is important to determine the distribution of americium in bone for both biokinetic information and also for the development of mathematical 'phantoms' which are close replicas of the amount of attenuation and scattering present in a human body. When a contaminated individual is screened for internally deposited gamma emitters, phantoms are used to assist in the calculation of how much activity is present. For instance, the attenuation factors for the Am-241 gammas in bone will have to take into account the time since exposure, because americium migrates from the endosteum outwards to the bone volume and the overall measured gamma signal will suffer less attenuation. 


\section{References}

[1] Shen G., Lee H.K., Hollow fiber-protected liquid-phase microextraction of triazine herbicides. Anal. Chem. 74 (2002) 648.

[2] Pedersen-Bjergaard S., Rasmussen K.E., Liquid-liquid-liquid microextraction for sample preparation of biological fluids prior to capillary electrophoresis. Anal. Chem. 71 (1999) 2650.

[3] Rezaee M., Yamini Y., Faraji M. Evolution of dispersive liquid-liquid microextraction method. J. Chromatogr. A. 1217 (2010). 16: 2342-2357

[4] Pena-Pereira F., Lavilla I, Bendicho C. Miniaturized preconcentration methods based on liquid-liquid extraction and their application in inorganic ultratrace analysis and speciation: A review. Spectrochim. Acta, Part B(2009). 64:1-15

[5] Chandrasekaran, K., Karunasagar D; Arunachalam J. Dispersive liquid-liquid micro extraction of uranium(VI) from groundwater and seawater samples and determination by inductively coupled plasma-optical emission spectrometry and flow injection-inductively coupled plasma mass spectrometry. Anal. Methods. 9 (2001). 3:2140-2147

[6] Khajeh, M, Nemch, TK, Pre-concentration of uranium from water samples by dispersive liquid-liquid micro-extraction .Radiochimica Acta. (2014). 102:887-893

[7] Sadi, Baki B.; Li, Chunsheng; Kramer, Gary H.; et al. Rapid determination of 226Ra in drinking water samples using dispersive liquid-liquid microextraction coupled with liquid scintillation counting. J Radioanal Nucl Chem (2011). 290:415-425

[8] Lester R. Morss (Editor), Norman Edelstein (Editor), Jean Fuger (Editor). (2010) The Chemistry of the Actinide and Transactinide Elements. Dordrect. Springer

[9] Cotton, Simon. (2006). Lanthanide and Actinide Chemistry. United States. John Wiley \& Sons Ltd.

[10] Jukka Lehto, Xiaolin Hou. (2011) Chemistry and Analysis of Radionuclides: Laboratory Techniques and Methodology. Weinheim. Wiley-VCH

[11] Eisenbud M, Gesell T (1997) Environmental radioactivity,4th ed. Academic Press, San Diego

[12] Warwick, P (2015) Environmental Radiochemical Analysis V. Croydon. Royal Society of Chemistry.

[13] Security of Radioactive Sources. (2003) International Atomic Agency Proceeding Series. Vienna. IAEA 
[14] Blake C., Baes Charles, Brown K. Solvent Extraction with Alkyl Phosphoric

Compounds . Ind. Eng. Chem., (1958), 50 12:1763-1767

[15] L’Annunziata, MF. Handbook of Radioactivity Analysis. Academic Press. 1998

[16] J.J. Fardy J.M. Chilton .Isolation of plutonium in chloride media-II: Solvent extraction with DI(2-ethylhexyl)phosphoric acid. J. Inorg. Nucl. Chem 31 (1969) $10: 3247-3254$

[17] H. Rameback, M. Sidlberg Separation of neptunium, plutonium, americium and curium from uranium with di-(2-ethylhexyl)-phosphoric acid (HDEHP) for radiometric and ICP-MS analysis. J. Radioanal. Nucl. Chem., 235 (1998) 12: 22 233

[18] Adloff, J. P. ; Guillaumont, R. (1993) Fundamentals of Radiochemistry; CRC Press: Boca Raton, FL,

[19] Maxwell, S.; Jones, V. Rapid determination of actinides in urine by inductively coupled plasma mass spectrometry and alpha spectrometry: A hybrid approach. Talanta (2009). 80:1: 143-150

[20] Hrnecek, E; Steier, P; Wallner, A Determination of plutonium in environmental samples by AMS and alpha spectrometry. Appl. Radiat. Isot. Volume: 63 (2005). 56:633-638

[21] Dai, X; Kramer-Tremblay, S. An emergency method for actinides in urine. Health Physic.(2011), 101:144-147

[22] Stather JW., Smith H., Bailey MR. The retention of C14 labelled DTPA in human volunteers after inhalation of intravenous injection. 44. (1983) 1:45-52

[23] Chang, H-S; Korshin, G; Ferguson, John. Investigation of mechanisms of oxidation of EDTA and NTA by permanganate at high pH. Environ. Sci. Technol. 40 (2006) 16: 5089-5094

[24] Tucker MD., Barton LL., Thomson, BM. Treatment of waste containing EDTA by chemical oxidation. Waste Management 19 (1999) 477-482

[25] Harley Ross. (1991) Liquid Scintillation Counting and Organic Scintillators. Chelsea. Lewis Publishers

[26] Michael Kessler. (2005). Liquid Scintillation Analysis - Science and Technology Perkin Elmer

[27] Knoll, G.F. (1999). Radiation Detection and Measurement (3rd ed.). Wiley. 
[28] Batten, JR. Evaluation of passivated ion implanted planar silicon detectors for the spectroscopy and assay of low energy electrons. Nucl. Instrum. Methods Phys. Res., Sect. A 277. (1989) 2-3:478-484

[29] Sill, CW., Williams, RL., Preparation of actinides for alpha spectrometry without electrodeposition. Analytical Chemistry. 53 (1981) 3:412-415

[30] Freeman, Arthur J. Lander, G. H. Keller, Cornelius (1984) Handbook on the physics and chemistry of the actinides. Amsterdam. North-Holland

[31] Li, Y; Peng, G; He, Q; et al. Dispersive liquid-liquid microextraction based on the solidification of floating organic drop followed by ICP-MS for the simultaneous determination of heavy metals in wastewaters Spectrochim. Acta, Part A 140 (2015). $156-161$

[32] Dacheux N., and Aupiais J., Determination of Uranium, Thorium, Plutonium, Americium, and Curium Ultratraces by Photon Electron Rejecting r Liquid Scintillation Analytical Chemistry, 69 (1997) 13

[33] Ramunasj M.,, Martell AE, Haye David The iron(II1)-catalyzedoxidationof EDTA in aqueous solution. Canadian Journal of Chemistry 58 (1980) 19

[34] Lloyd A. Currie. Limits for qualitative detection and quantitative determination. Application to radiochemistry Anal. Chem., (1968), 40 (3),586-593

[35] Li C, Vlahovich S, Dai X, Richardson RB, Daka JN, Kramer GH. Requirements for radiation emergency urine bioassay techniques for the public and first responders. Health Phys. (2010) 99(5):702-7

[36] Dantas B, Cardoso J, Tadei M, Juliao L, Lima M, Arine D, Ramos M, Alonso T, Lima F, Acar M, Dantas A. Current status of internal dosimetry service laboratories in Brazil. 2009 International Nuclear Atlantic Conference, Rio de Janerio, Brazil, September 27-October 2, 2009. Rio de Janeiro: Brasilian Nuclear Energy Association; 2009.

[37] Kumar R., Dubla, R, Yadav, JR. A method for estimation of Pu-isotopes in urine samples using TEVA resin and alpha spectrometry. J. Radioanal. Nucl. Chem. (2013) 295(3):2147-2152

[38] Annals of the ICRP Publication 103. The 2007 Recommendations of the International Commission on Radiological Protection

[39] R. W. Leggett and K. F. Eckerman (2003) Dosimetric Significance of the ICRP's Updated Guidance and Models, 1989-2003 and Implications for U.S. Federal Guidance. ORNL/TM-2003/207 
[40] Austin AL, Ellender M, Haines JW, J. Microdistribution and localized dosimetry of the a-emitting radionuclides $239 \mathrm{Pu}, 241 \mathrm{Am}$ and $233 \mathrm{U}$ in mouse femoral shaft int. Int $\mathrm{J}$ Radiat Biol, (2000)76:101=111

[41] P. L. Salmon,a Y. N. Onischuk,b O. A. Bondarenkob and L. E. Lanyonc, AlphaParticle Doses to Cells of the Bone Remodeling Cycle from Alpha-Particle-Emitting Bone-Seekers: Indications of an Antiresorptive Effect of Actinides Radiation Research 152 (1999), S43-S47

[42] Priest ND, Freemont A, Histopathology and 241Am microdistribution in skeletal USTUR case Skeletal case. Health Physics, 69 (1995) 3

[43] Lee, M. H.; Park, Y. J.; Jee, K. Y.; et al Study of an alpha track analysis and a fission track analysis for determining the hot particles contaminated with $\mathrm{Pu}$ and $\mathrm{U}$ isotopes. Appl. Radiat. Isot. 65 (2007) 1: 85-91

[44] Mitchell T., (2015) Introduction to anatomy and physiology musculoskeletal system. Wonder books.

[45] Carter, P. "Chapter 6: Bones and Skeletal Tissues." http://classes.midlandstech.edu/carterp/Courses/bio210/chap06/lecture1.html. Accessed Jun 1, 2016.

[46] Gregory R. Mundy M.D., Mr. Martin (1993) Physiology and Pharmacology of Bone-Springer-Verlag Berlin Heidelberg

[47] R. W. Leggett A retention-excretion mode for americium in humans Health Physics 62, (1992) 4

[48] Kathren RL., James F., McInroy Actinide distribution in the human skeleton Health physics Health Physics (1987) 52:179-192,

[49] Narbutt, J; Wodynski, A; Pecul, Ma The selectivity of diglycolamide (TODGA) and bis-triazine-bipyridine (BTBP) ligands in actinide/lanthanide complexation and solvent extraction separation - a theoretical approach. Dalton Transactions 44 (2015) 6: 26572666

[50] Ansari, S. A., Pathak, P., Mohapatra, P. K., Manchanda, V. K. Chemistry of Diglycolamides: Promising Extractants for Actinide Partitioning. Chemical Reviews, 112 (2011) 1751-1772.

[51] By: Maxwell, Sherrod L., III; Culligan, Brian K.; Noyes, Gary W. Rapid separation of actinides and radiostrontium in vegetation samples J. Radioanal. Nucl. Chem 286 (2010) $1273-282$ 
[52] Thakur, P.; Ballard, S.; Conca, J. L. Sequential isotopic determination of plutonium, thorium, americium and uranium in the air filter and drinking water samples around the WIPP site J. Radioanal. Nucl. Chem. 287 (2011) 1-311-321

[53] By: Sadi, Baki B.; Li, Chunsheng; Masoud, Ali; et al. An emergency bioassay method for Am-241 by extraction chromatography and liquid scintillation. Radiation Protection Dosimetry. 141 (2010) $2: 134-139$

[54] Novel extraction of chromatographic resins based on tetraalkyldiglycolamides:

Characterization and potential applications Horwitz, EP; McAlister, DR; Bond, AH; et al. Solvent Extraction and Exchange 23 (2005) 319-344

[55] Sasaki, U., Rapold, R., Arisaka, M., et. al. An Additional Insight into the Correlation between the distribution ratios and the aqueous acidity of the TODGA system. Solvent Extraction and Ion Exchange, 25 (2007) 187-204

[56] McInroy. J, Boyd H, Part IV: Preparation and analysis of tissue and bone. Health Physics 49 (1985) 4-587-621

[57] LaMont, SP; Glover, SE; Filby, RH Determination of plutonium-240/239 ratios in low activity samples using high resolution alpha-spectrometry J. Radioanal. Nucl. Chem. 234 (1998) 1-2 195-199

[58] Heymsfield, SB, Lohman TG., Wang, Z., Going, SB. (2005) Human Body Composition $-2^{\text {nd }}$ Edition. Human Kinetics. Champaign IL 


\section{Appendix:}

Bone Sample 1 (sub-samples \#1-18)

\begin{tabular}{|c|c|c|c|c|c|c|c|c|c|c|c|}
\hline $\begin{array}{l}\text { Sample } \\
\text { ID\# }\end{array}$ & dry (g) & $\operatorname{ash}(\mathrm{g})$ & $\begin{array}{l}\mathrm{Am} 243 \\
(\mathrm{~Bq})\end{array}$ & $\begin{array}{l}\text { Am243 } \\
\text { counts }\end{array}$ & $\begin{array}{l}\text { Am241 } \\
\text { counts }\end{array}$ & $\begin{array}{l}\text { Count } \\
\text { time } \\
(\mathrm{sec}) \\
\end{array}$ & $\begin{array}{l}\text { Am243 } \\
\text { (\% rec) }\end{array}$ & $\begin{array}{l}\mathrm{Am} 241 \\
(\mathrm{~Bq})\end{array}$ & $\begin{array}{l}\mathrm{Am} / \\
\text { Dry } \\
(\mathrm{Bq} / \mathrm{g})\end{array}$ & $\begin{array}{l}\mathrm{Am} / \\
\mathrm{Ash} \\
(\mathrm{Bq} / \mathrm{g})\end{array}$ & dry/ash \\
\hline 1 & 0.143 & 0.040 & 0.516 & 14558 & 2711 & 86400 & 0.157 & 0.200 & 1.391 & 5.054 & 0.275 \\
\hline 2 & 0.030 & 0.007 & 0.522 & 21628 & 1884 & 86400 & 0.231 & 0.094 & 3.155 & 12.711 & 0.248 \\
\hline 3 & 0.010 & 0.005 & 0.517 & 4884 & 185 & 604800 & 0.008 & 0.041 & 4.174 & 8.885 & 0.470 \\
\hline 4 & 0.016 & 0.008 & 0.514 & 16468 & 843 & 172800 & 0.089 & 0.055 & 3.458 & 6.860 & 0.504 \\
\hline 5 & 0.009 & 0.006 & 0.517 & 12615 & 346 & 86400 & 0.136 & 0.029 & 3.361 & 4.929 & 0.682 \\
\hline 6 & 0.008 & 0.006 & 0.512 & 19869 & 746 & 86400 & 0.216 & 0.040 & 4.776 & 6.629 & 0.720 \\
\hline 7 & 0.008 & 0.005 & 0.516 & 14121 & 387 & 172800 & 0.076 & 0.029 & 3.685 & 5.725 & 0.644 \\
\hline 8 & 0.032 & 0.018 & 0.518 & 14115 & 1563 & 86400 & 0.152 & 0.119 & 3.701 & 6.808 & 0.544 \\
\hline 9 & 0.014 & 0.008 & 0.250 & 19794 & 2571 & 172800 & 0.221 & 0.067 & 4.691 & 8.958 & 0.524 \\
\hline 10 & 0.016 & 0.010 & 0.247 & 18314 & 1457 & 172800 & 0.207 & 0.041 & 2.558 & 4.011 & 0.638 \\
\hline 11 & 0.019 & 0.013 & 0.250 & 17507 & 1326 & 172800 & 0.195 & 0.039 & 2.054 & 3.076 & 0.668 \\
\hline 12 & 0.019 & 0.011 & 0.250 & 22250 & 1576 & 172800 & 0.248 & 0.037 & 1.962 & 3.237 & 0.606 \\
\hline 13 & 0.044 & 0.030 & 0.249 & 2595 & 319 & 86400 & 0.058 & 0.064 & 1.452 & 2.144 & 0.677 \\
\hline 14 & 0.053 & 0.036 & 0.249 & 8726 & 1791 & 86400 & 0.195 & 0.106 & 2.015 & 2.960 & 0.681 \\
\hline 15 & 0.025 & 0.016 & 0.254 & 3244 & 465 & 86400 & 0.071 & 0.076 & 2.977 & 4.822 & 0.617 \\
\hline 16 & 0.077 & 0.045 & 0.252 & 5501 & 2466 & 86400 & 0.122 & 0.235 & 3.054 & 5.271 & 0.579 \\
\hline 17 & 0.070 & 0.049 & 0.243 & 7376 & 2214 & 172800 & 0.085 & 0.151 & 2.162 & 3.090 & 0.700 \\
\hline 18 & 0.013 & 0.012 & 0.249 & 15469 & 1645 & 172800 & 0.173 & 0.055 & 4.117 & 4.775 & 0.862 \\
\hline
\end{tabular}


Bone Sample 2 (sub-samples \#1-11)

\begin{tabular}{|c|c|c|c|c|c|c|c|c|c|c|}
\hline $\begin{array}{l}\text { Sample } \\
\text { ID\# }\end{array}$ & dry (g) & $\operatorname{ash}(\mathrm{g})$ & $\begin{array}{l}\mathrm{Am} 243 \\
(\mathrm{~Bq})\end{array}$ & $\begin{array}{l}\text { Am243 } \\
\text { counts }\end{array}$ & $\begin{array}{l}\text { Am241 } \\
\text { counts }\end{array}$ & $\begin{array}{l}\text { Am243 } \\
\text { (\% rec) }\end{array}$ & $\begin{array}{l}\mathrm{Am} 241 \\
(\mathrm{~Bq})\end{array}$ & $\begin{array}{l}\text { Am/ Dry } \\
(\mathrm{Bq} / \mathrm{g})\end{array}$ & $\begin{array}{l}\mathrm{Am} / \\
\text { Ash } \\
(\mathrm{Bq} / \mathrm{g})\end{array}$ & dry/ash \\
\hline 1 & 1.14 & 0.69 & 9.56 & 202497 & 98783 & 0.245 & 4.665 & 4.096 & 6.742 & 0.607 \\
\hline 2 & 2.07 & 1.27 & 9.59 & 193246 & 109099 & 0.233 & 5.414 & 2.615 & 4.279 & 0.611 \\
\hline 3 & 1.13 & 0.61 & 9.95 & 202116 & 62723 & 0.235 & 3.089 & 2.730 & 5.041 & 0.542 \\
\hline 4 & 1.22 & 0.70 & 9.95 & 203724 & 76597 & 0.237 & 3.739 & 3.077 & 5.318 & 0.579 \\
\hline 5 & 0.84 & 0.45 & 9.93 & 190544 & 44838 & 0.222 & 2.336 & 2.770 & 5.215 & 0.531 \\
\hline 6 & 0.80 & 0.34 & 9.52 & 160067 & 36701 & 0.195 & 2.183 & 2.717 & 6.486 & 0.419 \\
\hline 7 & 1.22 & 0.60 & 9.41 & 180673 & 87535 & 0.222 & 4.560 & 3.744 & 7.539 & 0.497 \\
\hline 8 & 1.15 & 0.56 & 9.84 & 200385 & 58226 & 0.236 & 2.860 & 2.492 & 5.094 & 0.489 \\
\hline 9 & 3.22 & 0.88 & 9.90 & 210368 & 84995 & 0.246 & 4.001 & 1.241 & 4.555 & 0.272 \\
\hline 10 & 0.97 & 0.43 & 9.92 & 198022 & 35234 & 0.231 & 1.765 & 1.821 & 4.080 & 0.446 \\
\hline 11 & 3.68 & 2.27 & 10.09 & 203839 & 206943 & 0.234 & 10.243 & 2.787 & 4.516 & 0.617 \\
\hline
\end{tabular}

Note $:$ Count time $=86400$ s for all samples 